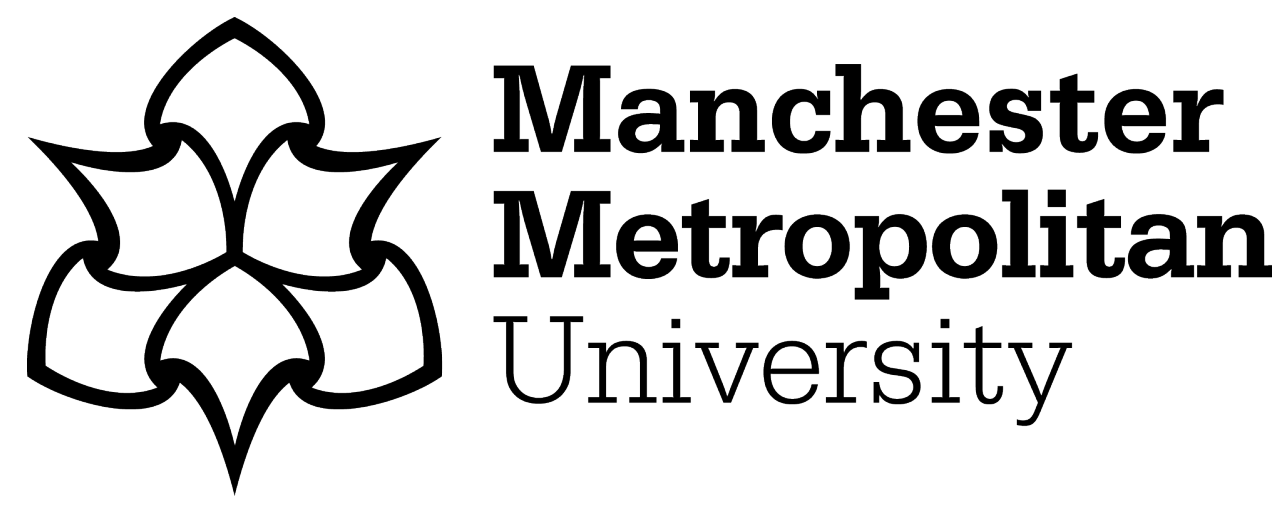

Fagan, Vincent, Johansson, Catrine, Gileadi, Carina, Monteiro, Octovia, Dunford, James Edward, Nibhani, Reshma, Philpott, Martin, Malzahn, Jessica, Wells, Graham, Farham, Ruth, Cribbs, Adam, Halidi, Nadia, Li, Fengling, Chau, Irene, Greschik, Holger, Velupillai, Srikannathasan, AllaliHassani, Abdellah, Bennett, James M, Christott, Thomas, Giroud, Charline, Lewis, Andrew M ORCID logoORCID: https://orcid.org/0000-0002-6447354X, Huber, Kilian VM, Athanasou, Nick, Bountra, Chas, Jung, Manfred, Schüle, Roland, Vedadi, Masoud, Arrowsmith, Cheryl H, Xiong, Yan, Jin, Jian, Fedorov, Oleg, Farnie, Gillian, Brennan, Paul E and Oppermann, Udo CT (2019) A Chemical Probe for Tudor Domain Protein Spindlin1 to Investigate Chromatin Function. Journal of Medicinal Chemistry, 62 (20). pp. 9008-9025. ISSN 0022-2623

Downloaded from: https://e-space.mmu.ac.uk/624967/

Version: Accepted Version

Publisher: American Chemical Society

DOI: https://doi.org/10.1021/acs.jmedchem.9b00562

Please cite the published version 


\title{
A Chemical Probe for Tudor Domain Protein Spindlin1 to Investi- gate Chromatin Function
}

\author{
Vincent Fagan, ${ }^{\dagger}, \Delta, \pitchfork$ Catrine Johansson, ${ }^{\dagger}, \gamma,{ }^{\ddagger}$ Carina Gileadi, ${ }^{\dagger}, \gamma$ Octovia Monteiro, ${ }^{\dagger, \Delta}$ James Dunford, ${ }^{\gamma}$ \\ Reshma Nibhani, ${ }^{\gamma}$ Martin Philpott, ${ }^{\gamma}$ Jessica Malzahn, ${ }^{\gamma}$ Graham Wells, ${ }^{\gamma}$ Ruth Farham, ${ }^{\gamma}$ Adam Cribbs, ${ }^{\gamma}$ \\ Nadia Halidi, ${ }^{\dagger}, \gamma$ Fengling Li, ${ }^{\psi}$ Irene Chau, ${ }^{\psi}$ Holger Greschik, ${ }^{\infty}$ Srikannathasan Velupillai, ${ }^{\dagger}$ Abdellah Al- \\ lali-Hassani, ${ }^{\psi}$ James Bennett, ${ }^{\dagger, \Delta}$ Thomas Christott, ${ }^{\dagger, \Delta}$ Charline Giroud, ${ }^{\dagger, \Delta}$ Andrew M. Lewis, ${ }^{\dagger, \Delta}$ Kilian \\ V.M. Huber, ${ }^{\dagger, \Delta}$ Nick Athanasou, ${ }^{\gamma}$ Chas Bountra, ${ }^{\dagger}$ Manfred Jung, ${ }^{\S, \infty}$ Roland Schüle, ${ }^{\infty}$ Masoud Vedadi, ${ }^{\psi}$ \\ Cheryl Arrowsmith, ${ }^{\psi}$ Yan Xiong, ${ }^{\perp}$ Jian Jin, ${ }^{\perp}$ Oleg Fedorov,${ }^{\dagger, \Delta}$ Gillian Farnie, ${ }^{\dagger, \gamma}$ Paul E. Brennan, ${ }^{*, \dagger, \Delta, \nabla}$ \\ Udo Oppermann $*, \dagger, \gamma, \S$
}

${ }^{\dagger}$ Structural Genomics Consortium, Nuffield Department of Medicine, University of Oxford, OX3 7DQ, Oxford, UK.
${ }^{\Delta}$ Target Discovery Institute, Nuffield Department of Medicine, University of Oxford, OX3 7FZ, Oxford, UK.

'Botnar Research Centre, Nuffield Department of Orthopaedics, Rheumatology and Musculoskeletal Sciences, NIHR Biomedical Research Centre, University of Oxford, Oxford, OX3 7LD, UK.

${ }^{\psi}$ Structural Genomics Consortium, University of Toronto, 101 College Street, Toronto, ON M5G 1L7, Canada.

${ }^{\infty}$ Department of Urology, Center for Clinical Research, University Freiburg Medical Center, University of Freiburg, D-79106 Freiburg, Germany.

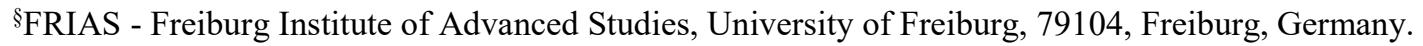

${ }^{\perp}$ Mount Sinai Center for Therapeutics Discovery, Departments of Pharmacological Sciences and Oncological Sciences, Tisch Cancer Institute, Icahn School of Medicine at Mount Sinai, New York, NY 10029, USA.

${ }^{\nabla}$ Alzheimer's Research UK Oxford Drug Discovery Institute, Nuffield Department of Medicine, University of Oxford, OX3 7FZ, Oxford, UK.

Supporting Information Placeholder

\begin{abstract}
Modifications of histone tails, including lysine/arginine methylation, provide the basis of a 'chromatin or histone code'. Proteins that contain 'reader' domains can bind to these modifications and form specific effector complexes, which ultimately mediate chromatin function. The spindlin1 (SPIN1) protein contains three Tudor methyllysine/arginine reader domains and was identified as a putative oncogene and transcriptional co-activator. Here we report a SPIN1 chemical probe inhibitor with low nanomolar in vitro activity, exquisite selectivity on a panel of methyl reader and writer proteins, and with submicromolar cellular activity. X-ray crystallography showed that this Tudor domain chemical probe simultaneously engages Tudor domains 1 and 2 via a bidentate binding mode. Small molecule inhibition and siRNA knockdown of SPIN1, as well as chemoproteomic studies, identified genes which are transcriptionally regulated by SPIN1 in squamous cell carcinoma and suggest that SPIN1 may have a roll in cancer related inflammation and/or cancer metastasis.
\end{abstract}

\section{INTRODUCTION}

Epigenetic methyllysine and arginine reader proteins play a crucial role in many biological processes. They bind to posttranslational methylation marks on histone tails, thus interpreting and conveying the "histone or chromatin code" downstream. ${ }^{1-2}$ This large family of proteins consists of Chromo, PHD, Tudor, MBT and PWWP domains. ${ }^{3}$ Within the Tudor domains, there are five human spindlin proteins (SPIN1, 2A, 2B, 3 and 4) and each contain three Tudor methyl reader domains. ${ }^{4-9}$ Spindlin1 (SPIN1) was initially identified in mouse oocytes, ${ }^{4}$ but has now been implicated in many cancers. ${ }^{7,}{ }^{10-18}$ SPIN1 binds to trimethylated lysine 4 of histone 3 (H3K4me3) via the "aromatic cage" of Tudor domain $2 .{ }^{17,}{ }^{19-20}$ It can also simultaneously bind to the asymmetrically dimethylated arginine mark H3R8me2a via domain $1,{ }^{17}$ which makes SPIN1 a bivalent methyllysine and methylarginine reader. SPIN1 also binds to H4K20me3 via domain 2, but little is known about this interaction. ${ }^{21}$ Binding to these epigenetic marks at gene promoter regions leads to transcriptional activation of ribosomal proteins ${ }^{20}$ and promotes liposarcoma, ovarian, colon, breast and non-small-cell lung cancers by activation of the GDNF-RETMAZ, ${ }^{7}$ Wnt $/ \beta$-catenin, ${ }^{17-18}$ or PI3K/Akt ${ }^{11,15-16}$ pathways respectively. SPIN1 has also been shown to facilitate the inactivation of $\mathrm{p} 53$, through interaction with the ribosomal protein $\mathrm{uL} 18 .{ }^{14} \mathrm{Re}-$ cently the C11ORF84 protein was identified that attenuates SPIN1 coactivator activity. ${ }^{22}$ Knockdown of SPIN1 resulted in cancer cell and xenograft tumor growth inhibition, which suggests that small molecule inhibition of SPIN1 may be a viable approach for the treatment of certain cancers., ${ }^{71-15}$ Therefore, potent and selective SPIN1 inhibitors are needed to aid with the further elucidation of SPIN1 biology. Micromolar inhibitors were identified using in silico methods ${ }^{23}$ and bidentate inhibitors that interact with Tudor domains 1 and 2 were reported. ${ }^{5}$ 


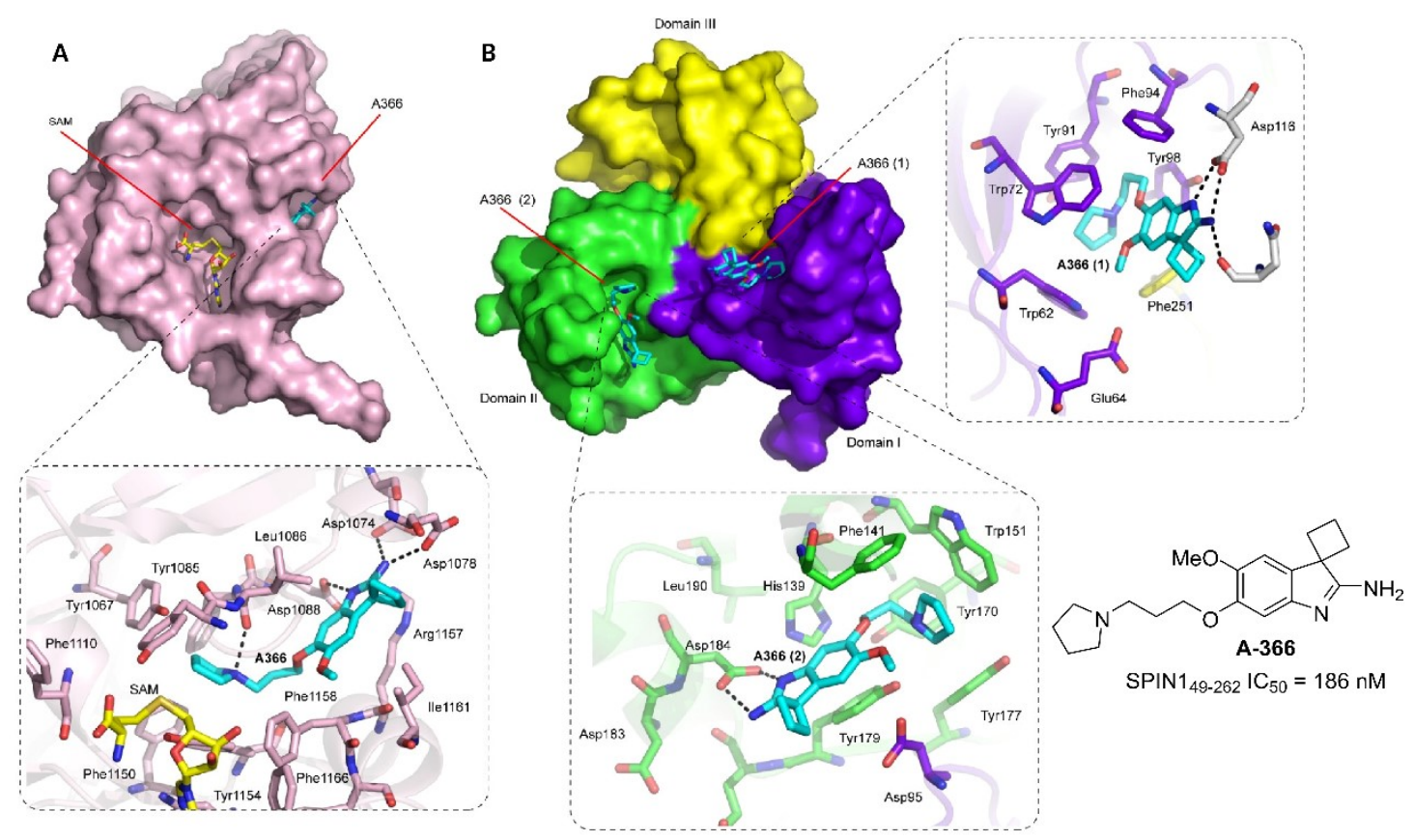

Figure 1. Crystal structure of A-366 with G9a and SPIN1. (A) Structural of A-366 (cyan) in complex with G9a (pink) (PDB ID 4NVQ). ${ }^{24}$ (B) Structure of A-366 (cyan) in complex with SPIN1 (PDB ID 6I8Y).

However, with binding affinities above $3 \mu \mathrm{M}$, these inhibitors have considerably weaker potency than that required for chemical probe inhibitors (in vitro $\mathrm{K}_{\mathrm{D}}<100 \mathrm{nM}$ ). ${ }^{25-26}$ We recently identified a potent SPIN1 hit molecule A-366 (Figure 1, IC $50186 \mathrm{nM}$ ). ${ }^{27}$ However this molecule is a potent chemical probe for the methyltransferase $\mathrm{G} 9 \mathrm{a}\left(\mathrm{IC}_{50} 3 \mathrm{nM}\right){ }^{24}$ Using A-366 as a starting point, we set out to develop SPIN1 inhibitors and we now report the biochemical, structural and cellular characterization that led to a potent and selective SPIN1 chemical probe.

\section{Results}

We initially used X-ray crystallography to compare the binding modes of A-366 in complex with SPIN1 and G9a. Binding of A366 to G9a is largely dominated by polar contacts (Figure 1A). ${ }^{24}$ While in SPIN1, interestingly, two molecules of A-366 bind, one in each aromatic cage of domain 1 and domain 2 (Figure 1B). In domain 1 , binding is largely driven by $\pi$-stacking interactions with residues Trp62, Trp72, Tyr91, Phe94 and Tyr98, and in domain 2 via contacts with residues His139, Phe141, Trp151, Tyr177 and Tyr179, as well as, polar interactions with Asp184. For inhibitor design we focused on domain 2 of SPIN1, since binding of the $\mathrm{H} 3_{1-10} \mathrm{~K} 4 \mathrm{me} 3-\mathrm{R} 8 \mathrm{me} 2 \mathrm{a}$ peptide to SPIN1 is largely driven by the interaction of $\mathrm{K} 4 \mathrm{me} 3$ with domain $2 .{ }^{17}$ Compared to G9a, domain 2 of SPIN1 can accommodate larger substituents. Therefore, the chemical synthesis of $\mathrm{A}-366^{24}$ was modified to allow incorporation of larger alkyl groups at $\mathrm{R}^{1}$ and optimized to allow efficient incorporation of tert-amines at $\mathrm{R}^{2}$ (Scheme 1, Series $1 \& 2$ )

Commercially available compound $\mathbf{1}$ was dialkylated using the procedure of Barbasiewicz et al, with slight modification, to incorporate a spirocycle (compound 2), which in later analogues, was replaced with gem-dimethyls (compound 3). A selective nitration was achieved at low temperature, which was followed by debenzylation by hydrogenolysis to give the nitrophenol $\mathbf{6}$. The solvent mixture (9:1 EtOAc:EtOH) was decisive in determining the outcome of this reaction, with most other solvent mixtures leading to significant reduction of the nitro group. Nitrophenol 6 was alkylated to give compound 7 and the chloride was displaced with pyrrolidine to give compound $\mathbf{8}$. The demethylation of $\mathbf{8}$ was achieved by combining aspects of the procedures reported by Chae et al. and Magano et al, which involved nucleophilic displacement of the phenoxide by an in situ generated thiolate. Demethylation via a lewis acid (e.g. $\mathrm{BBr}_{3}$ ) led to mixtures of phenols 6 and 9. Alkylation of phenol 9 with bulkier alkylhalides was followed by the nitro group reduction with zinc in acetic acid. The resultant aniline underwent in situ ring closure to give a series of cyclic amidines possessing various alkyl groups at $\mathrm{R}^{1}$. For the preparation of series 2 , the chloride of compound 7 was displaced with various secondary amines, followed by reductive ring closure.

An AlphaScreen assay was employed to assess the SPIN1 binding of the compounds. Compound binding was determined by the displacement of a trimethylated biotinylated histone 3 peptide (ARTK(me3)QTARKSTGGKAPRK-biotin) from a His tagged SPIN1 protein using an AlphaScreen ${ }^{\circledR}$ Histidine (Nickel Chelate) Detection Kit. The scintillation proximity assay (SPA) reported by Sweis et al. was used to evaluate the G9a inhibition. ${ }^{24}$

A methylcyclopropyl group at $\mathrm{R}^{1}$ (compound 10) imparted SPIN1 selectivity, while isoindolines at $\mathrm{R}^{2}$ (e.g. compound 11) increased the binding affinity. These optimum substituents were incorporated into series 3 (Scheme 1). To prepare series 3, general conditions (d), (e), (f), (g) and (i) were employed, but the order of cleavage of the benzyl- and methyl-arylethers was reversed. Series 3 contained potent SPIN1 inhibitors (Table 1, compound 22, 23, 25). Compound 25, which had gem-dimethyls in place of a spirocycle, was equipotent as a SPIN1 inhibitor but showed no inhibition of G9a, which indicated that the spirocycle was not required. Compounds possessing butyl chains had dramatically lower potency and therefore, were used as inactive controls (Table 1, compound 24, 26). The inactive control compound 26 had a 19fold decrease in potency compared to its corresponding active compound 25. Unexpectedly, the use of a slightly longer SPIN1 construct resulted in a higher $\mathrm{IC}_{50}$ for A-366 (Table 1) than that previously reported, ${ }^{27}$ which was explored later. 
Scheme 1. Synthesis of SPIN1 inhibitors Series 1, 2 and 3.

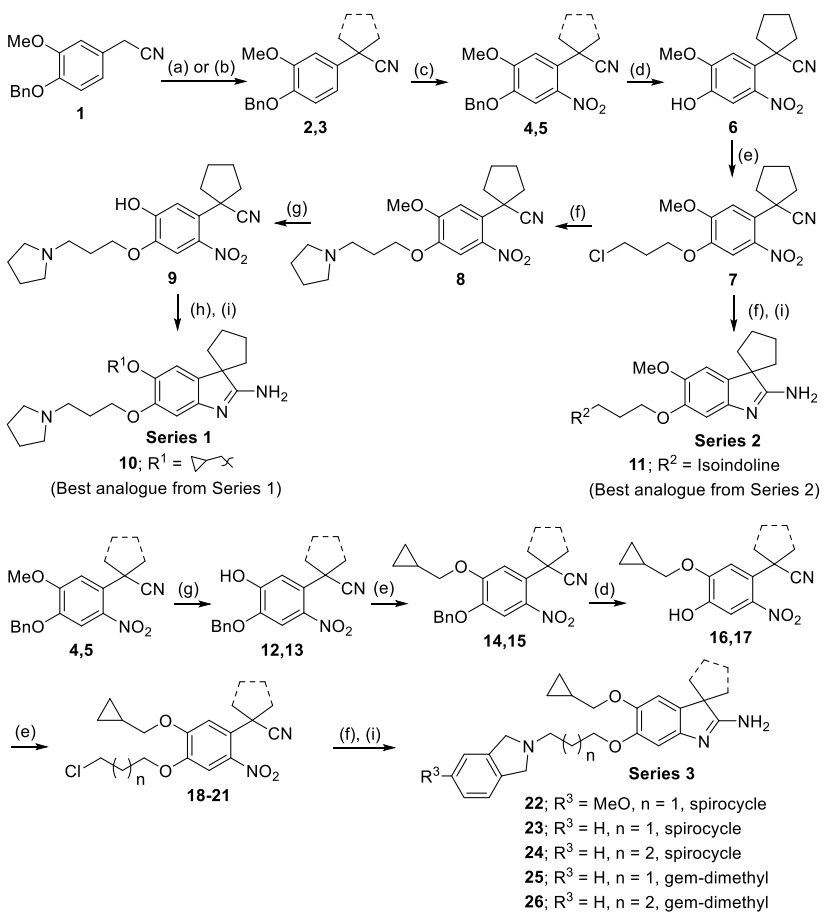

General conditions: (a) 1,4-dibromobutane, $\mathrm{KOH}, \mathrm{TBAB}, \mathrm{PhMe}$, $0{ }^{\circ} \mathrm{C}$-reflux, 3h, 92\%; (b) NaOH, MeI, DMSO, rt, 1 h 98\%; (c) $\mathrm{HNO}_{3}, \mathrm{Ac}_{2} \mathrm{O}, \mathrm{AcOH},-5{ }^{\circ} \mathrm{C}, 1 \mathrm{~h}, 87-92 \%$; (d) $\mathrm{Pd} / \mathrm{C}, \mathrm{H}_{2}, 9: 1$ EtOAc:EtOH, rt, 30-40 min, 76-86\%; (e) alkylbromide, $\mathrm{K}_{2} \mathrm{CO}_{3}$, $\mathrm{CH}_{3} \mathrm{CN}, 80{ }^{\circ} \mathrm{C}, 18 \mathrm{~h}, 92-97 \%$; (f) $2^{\circ}$ amine, $\mathrm{KI}, \mathrm{K}_{2} \mathrm{CO}_{3}, \mathrm{CH}_{3} \mathrm{CN}$, $80{ }^{\circ} \mathrm{C}, 48 \mathrm{~h}$; (g) $\mathrm{C}_{12} \mathrm{H}_{25} \mathrm{SH}, \mathrm{KOtBu}, \mathrm{DMF}, 0-50{ }^{\circ} \mathrm{C}, 2 \mathrm{~h}, 79-86 \%$; (h) alkylhalide, $\mathrm{K}_{2} \mathrm{CO}_{3}, \mathrm{DMF}, 50^{\circ} \mathrm{C}, 18 \mathrm{~h}$; (i) $\mathrm{Zn}, \mathrm{AcOH}, 110{ }^{\circ} \mathrm{C}$, $2 \mathrm{~h}, 5-82 \%$.

Table 1. Compound IC50 on SPIN1 (AlphaScreen assay) and G9a (Scintillation Proximity Assay)

\begin{tabular}{ccc}
\hline Compound & $\begin{array}{c}\text { SPIN1 49-262 } \text { IC }_{50} \\
{[\boldsymbol{\mu M}]} \\
\text { (hill slope, SD) }\end{array}$ & $\begin{array}{c}\text { G9a IC50 } \\
{[\boldsymbol{\mu M}] \text { (hill slope) }}\end{array}$ \\
\hline $\mathbf{1 0}$ & $1.12(1.0,0.30)$ & $3.1(0.8)$ \\
$\mathbf{1 1}$ & $0.66(0.8,0.46)$ & $10(0.7)$ \\
$\mathbf{2 2}$ & $0.34(1.0,0.21)$ & Not determined \\
$\mathbf{2 3}$ & $0.39(1.0,0.25)$ & No inhibition \\
$\mathbf{2 4}$ & $6.22(0.8,3.20)$ & No inhibition \\
$\mathbf{2 5}$ & $0.36(0.8,0.06)$ & No inhibition \\
$\mathbf{2 6}$ & $6.92(0.7,0.88)$ & No inhibition \\
\hline $\mathbf{A - 3 6 6}$ & 1.5 & $0.003{ }^{\mathrm{b}}$ \\
\hline${ }^{\mathrm{a}} \mathrm{n} \geq 2 ;{ }^{\mathrm{b}}$ Sweis et al. ${ }^{24}$ & &
\end{tabular}

The crystal structure of compound 23 in complex with SPIN1 (SI, Figure S1) suggested that the improved binding affinity of isoindoline-containing analogues may be attributed to additional $\pi$-stacking interactions between the isoindoline moiety and residues Phe141, Trp151, Tyr170 and Tyr177 in the aromatic cage.

With potent compounds in hand, cellular target engagement was assessed using a nanoBRET assay. ${ }^{28}$ A halotag-histone 3.3 and an N-terminal Nanoluc-SPIN1 fusion protein was overexpressed in U2OS cells and treated with compounds 23-26 at a concentration of $1 \mu \mathrm{M}$. A significant reduction of the nanoBRET signal was observed for compound $\mathbf{2 3}$ and $\mathbf{2 5}$, but not for the inac- tive 24 and 26, which confirmed that the inhibitors indeed showed cellular target engagement (Figure 2). However, at elevated concentrations $(>3 \mu \mathrm{M})$ increased cell death was observed (SI, Figure S6). Both the active and inactive compounds displayed this toxicity, which implicated off-target effects. Despite significant effort, the toxicity issues relating to this series could not be resolved.

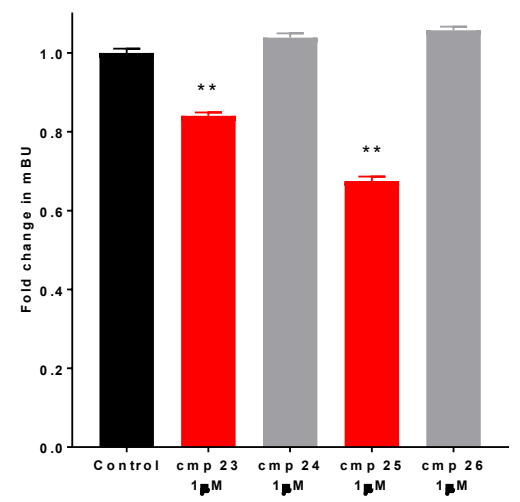

Figure 2. NanoBRET assay. Full length wild type SPIN1 and Histone3.3 nanobret assay showing in cell target engagement for compound 23 and 25. Graph shows fold change in milliBRET Units (mBU) after $24 \mathrm{~h}$ treatment with compounds at $1 \mu \mathrm{M}$. Mean $\pm \mathrm{SD}, \mathrm{n}=3$ independent experiments, $\mathrm{n} \geq 3$ internal replicates. One way ANOVA with Dunnetts post-hoc multiple comparison test, $* * \mathrm{p}<0.005$.

Recently bidentate inhibitors were reported that engaged both domain 1 and 2 of SPIN1, and although they had weak affinity, they showed a good degree of selectivity. ${ }^{5}$ Therefore, in efforts to increase the SPIN1 selectivity and binding affinity, and to overcome the toxicity issues, bidentate inhibitors were designed. We aimed to link compound $\mathbf{2 5}$ to the moiety report by Bae et al. ${ }^{5}$ which binds to domain 1 of SPIN1 (Scheme 2, highlighted in blue). The initial use of simple alkyl chain linkers resulted in highly flexible molecules, which did not engage with two SPIN1 domains. Therefore, the Huisgen copper catalyzed azide-alkyne cycloaddition (CuAAC) reaction was utilized since it resulted in more rigid and hydrophilic triazole-containing linkers. It also allowed the linker length to be varied easily. Alkynefunctionalized isoindolines $\mathbf{2 7}$ and $\mathbf{2 8}$ (Scheme 2) were prepared by $O$-alkylation of the corresponding $N$-Boc protected phenol, followed by Boc deprotection. Alkylation at the 5-position of these isoindolines was chosen, since earlier structure-activity relationship studies revealed that substitution at this position was well tolerated (e.g. Scheme 1, compound 22). Using general conditions (f), isoindoline 27 and 28 were coupled to 19 and 21 to give compounds 29-31, which were subjected to the $\mathrm{CuAAC}$ reaction (general conditions (j)) with azide-functionalized $\mathbf{3 2}$ and 33, using copper powder as catalyst (Scheme 2). Reductive cyclization using general conditions (i) afforded compounds 34-38. Azide-functionalized $\mathbf{3 2}$ and $\mathbf{3 3}$ were prepared by amide coupling of commercially available 4-(2-(Pyrrolidin-1-yl)ethyl)piperidine with the corresponding azido carboxylic acids. The bidentate compounds 34-38, as well as compound 25, were evaluated as SPIN1 inhibitors using a range of biophysical assays (Table 2). Three of these compounds displayed a large increase in potency compared to $\mathbf{2 5}$. Compound $\mathbf{3 5}$ was chosen as a chemical probe candidate since it displayed marginally better results in three of the four assays. Based on the isothermal titration calorimetry (ITC) alone, there was little to distinguish between compounds 35-37. 
Scheme 2. Synthesis of bidentate SPIN1 inhibitors.

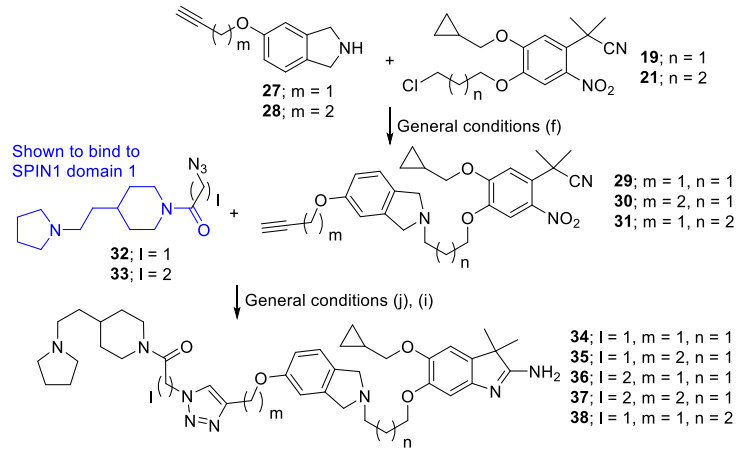

General conditions: (f) $2^{\circ}$ amine, $\mathrm{KI}, \mathrm{K}_{2} \mathrm{CO}_{3}, \mathrm{CH}_{3} \mathrm{CN}, 80{ }^{\circ} \mathrm{C}, 48$ h; (j) $\mathrm{Cu}, \mathrm{DMF}, 60^{\circ} \mathrm{C}, 2 \mathrm{~h}$; (i) $\mathrm{Zn}, \mathrm{AcOH}, 110^{\circ} \mathrm{C}, 2 \mathrm{~h}, 5-82 \%$.

Table 2. Biophysical assays of bidentate SPIN1 inhibitors.

\begin{tabular}{|c|c|c|c|c|}
\hline Cmp & $\begin{array}{l}\text { AlphaScreen } \\
\text { SPIN1 26-262 IC50 [nM] } \\
\text { (hill slope, } \text { SD) }^{\mathrm{a}}\end{array}$ & $\begin{array}{c}\text { Octet BLI } \\
\text { SPIN1 } 21-262 \\
\text { KD [nM] }\end{array}$ & $\begin{array}{c}\text { ITC } \\
\text { SPIN1 } 1_{49-262} \\
\text { KD }_{\mathrm{D}}[\mathrm{nM}]\end{array}$ & $\begin{array}{c}\text { Tm Shift } \\
\text { SPIN1 }{ }_{26-262} \\
\Delta \mathrm{T}_{\mathrm{m}}\left[{ }^{\circ} \mathrm{C}\right]\end{array}$ \\
\hline 25 & $362(0.8,0.06)$ & 94 & 15.4 & $6.8 \pm 0$ \\
\hline 34 & $152(1.4,0.12)$ & 400 & 60 & nd \\
\hline 35 & $33(2.3,0.01)$ & 55 & 9.9 & $13.2 \pm 0.3$ \\
\hline 36 & $48(1.4,0.03)$ & 67 & 6.6 & $12.8 \pm 0.2$ \\
\hline 37 & $59(1.8,0.04)$ & 72 & 10.1 & $11.0 \pm 0$ \\
\hline 38 & $3388(0.7,1.24)$ & nd & 1300 & $1.0 \pm 0$ \\
\hline
\end{tabular}

Calorimetry), nd (not determined)

It is worth noting that compound $\mathbf{3 5}$ is considerably more potent as a SPIN1 inhibitor than those reported previously. ${ }^{5}$

Compound 38 was prepared as an inactive control. ITC showed that $\mathbf{3 8}$ was approx. 130 times less potent than $\mathbf{3 5}$ and thus, compounds 35 and 38 represent good candidates for an active and inactive chemical probe pairing (CPP)

The crystal structure of $\mathbf{3 5}$ in complex with SPIN1 confirmed a bidentate binding mode, where the ethylpyrrolidine moiety indeed occupies the aromatic cage of domain 1, while binding of the isoindoline to domain 2 is retained (Figure 3 ).

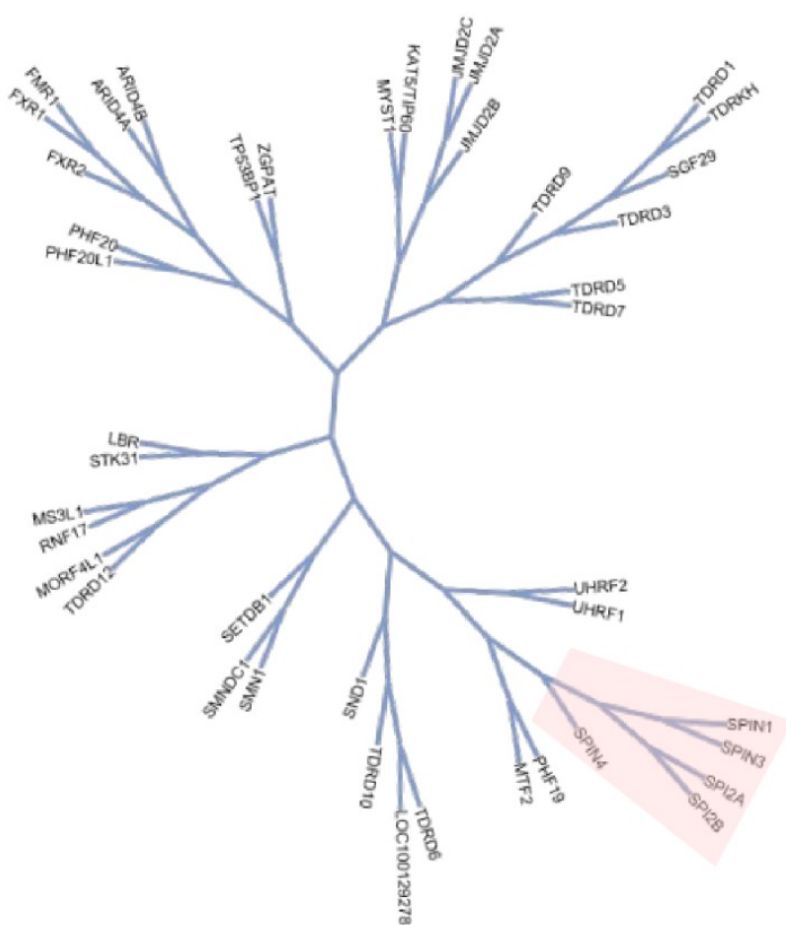

Figure 4. Human Tudor Domain Phylogenetic tree with SPIN subfamily highlighted.

Table 3. Average ITC $K_{D}(n M)$ of 35 on human SPIN subfamily members.

\begin{tabular}{llllll}
\hline Cmp & SPIN1 49-262 & $\begin{array}{l}\text { SPIN1 26- } \\
\text { 262 }\end{array}$ & SPIN2B $_{\text {45-258 }}$ & $\begin{array}{l}\text { SPIN327- } \\
\text { 258 }\end{array}$ & SPIN4 36-249 \\
\hline 35 & 9.9 & 111.1 & 46.1 & 131.1 & 18.1 \\
\hline
\end{tabular}

There are 5 distinct human SPIN family members which form a distinct branch of the Tudor domain phylogenetic tree (Figure 4). The selectivity of $\mathbf{3 5}$ was assessed by ITC on four SPIN subfamily members (Table 3 ). SPIN2A was omitted due to its similarity to SPIN2B and its low endogenous expression. The results showed that compound $\mathbf{3 5}$ binds with varying affinity across the subfamily $\left(\mathrm{K}_{\mathrm{D}} 10-130 \mathrm{nM}\right)$. This suggests a similar binding mode among the SPIN proteins and is in line with their overall structures and peptide binding features (SI, Figure S2).
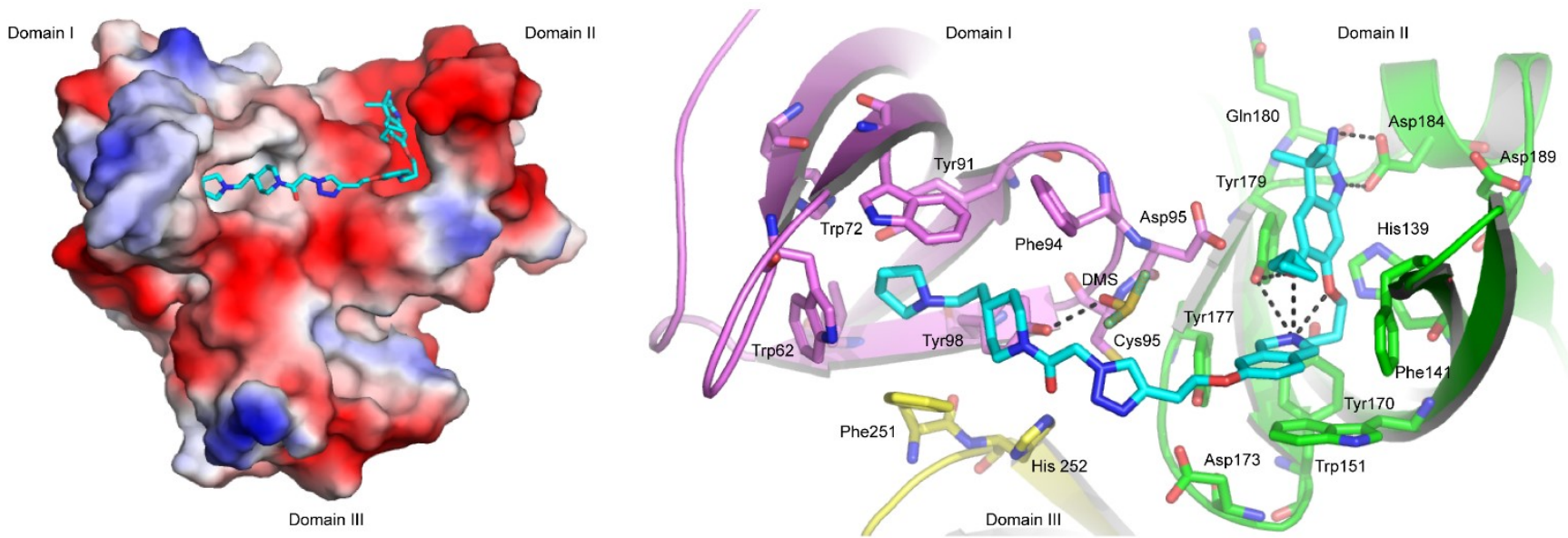

Figure 3. Crystal structure of SPIN1 ${ }_{49-262}$ in complex with compound 35 (PDB ID 6I8B). Left, electrostatic surface potential of SPIN1 with 35. Right, interactions of 35 (cyan) with SPIN1 (domains 1, 2 \& 3 are shown in magenta, green \& yellow respectively). 
In order to investigate the discrepancies between the SPIN1 $\mathrm{IC}_{50}$ of A-366 observed here (Table 1) versus that reported previously, ${ }^{27}$ the ITC of compound $\mathbf{3 5}$ was performed using two SPIN1 constructs (Table 3). SPIN126-262 was used here in the AlphaScreen assay, while SPIN149-262 was employed in the previous study. ${ }^{27}$ The use of a SPIN1 construct ranging from residues 49262 resulted in a 10 - to 15 -fold lower $K_{D}$ compared to that obtained with the longer construct of residues 26-262, which is in agreement with the previous study.

Further investigation into the influence of the protein construct on ligand binding was deemed unnecessary, since the nanoBRET cellular target engagement assay employed here, used full length SPIN1. Compound 35 displayed potent cellular SPIN1 inhibition (Figure 5) and with an $\mathrm{EC}_{50}$ of $270 \mathrm{nM}$, it has cellular potency well within the acceptable range for a chemical probe. ${ }^{25-26}$ The inactive control compound $\mathbf{3 8}$ displayed no inhibition and crucially, nonspecific toxicity was not observed for $\mathbf{3 5}$ or $\mathbf{3 8}$.
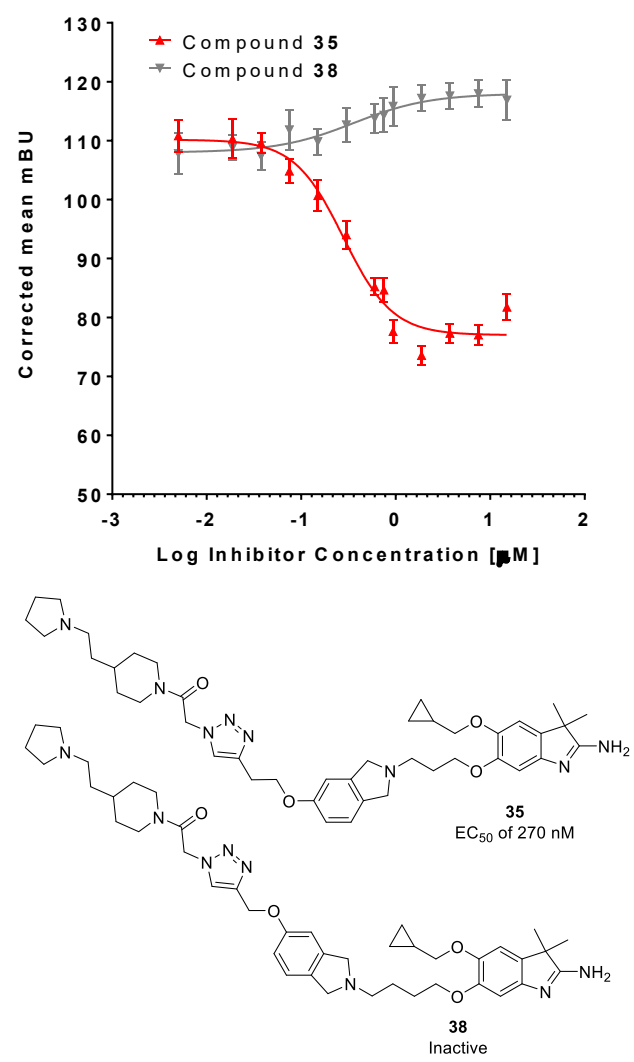

Figure 5. NanoBRET assay results for compound $\mathbf{3 5}$ and $\mathbf{3 8}$ using full length wild-type SPIN1 \& Histone 3.3. Graph shows milliBRET Units (mBU) after $24 \mathrm{~h}$ treatment with $0.05-15 \mu \mathrm{M}$ of compound $\mathbf{3 5}$ and $\mathbf{3 8}$.

Compound 35 and 38 were screened against methyl-Lys/Arg binding domains (including Chromo, PHD, Tudor and MBT domains) using a SYPRO Orange thermal shift assay (DSF (Differential Scanning Fluorimetry)). ${ }^{29}$ In agreement with the ITC data, compound 35 induced a large shift in the thermal stability of all SPIN proteins assessed $\left(\Delta \mathrm{T}_{\mathrm{m}} 6.5-14.1{ }^{\circ} \mathrm{C}\right.$, Figure 6$)$. No other significant thermal shift was observed for compound $\mathbf{3 5}$ or $\mathbf{3 8}$.

Compound 35 and 38 were also tested against 33 protein, DNA or RNA methyltransferases using established enzymatic SPAs as described previously. ${ }^{30}$ The compounds were initially tested at high concentration (10 and $50 \mu \mathrm{M}$, Figure 6) and where inhibition occurred,
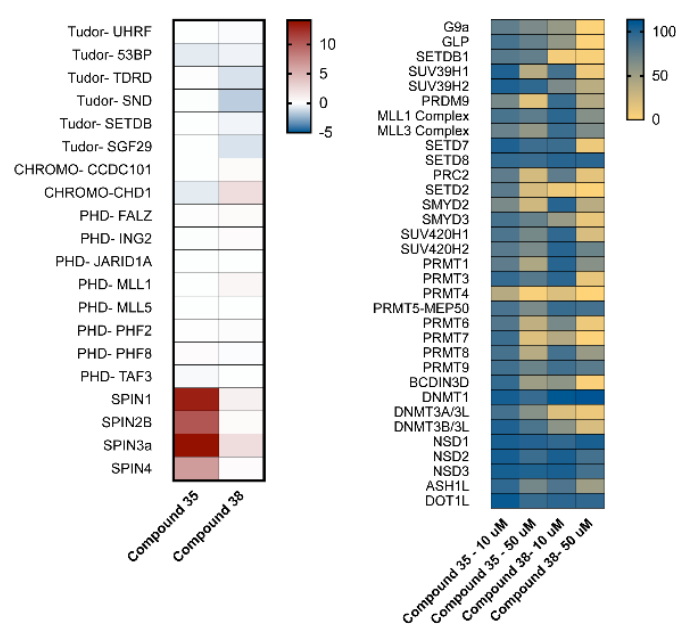

Figure 6. (LEFT) Heatmap of selected methyl-Lys/Arg reader domains using DSF (thermal shift assay). Scale shows $\Delta \mathrm{T}{ }^{\circ} \mathrm{C}$. (RIGHT) Percentage enzyme activity of 33 methyltransferases after compound treatment at 10 and $50 \mu \mathrm{M}$.

$\mathrm{IC}_{50}$ values were determined (SI, Table S4). Compound 35 displayed at least 300-fold selectivity over all methyltransferases tested.

In addition, a fluorescence polarization assay was employed to test compound $\mathbf{3 5}$ and $\mathbf{3 8}$ against the malignant brain tumor methyl readers, L3MBTL1 and L3MBTL3. The compounds showed no significant inhibition and compound $\mathbf{3 5}$ had approximately 250-fold higher potency towards SPIN1 than towards these MBT methyl readers (SI, Figure S5).

Chemoproteomics was employed to identify cellular interacting partners of compound 35. ${ }^{31}$ Compound 41 was prepared by subjecting isoindoline 39 and chloride 19 to general conditions (f) and (i) to give the benzyl protected 40. Hydrogenolysis, followed by alkylation with 4-(Boc-amino)butyl bromide, and Boc deprotection, gave the amino functionalized 41 (Scheme 3 ). The affinity probe 41 was coupled to NHS-activated sepharose beads and incubated with SCC040 cell lysates in the presence of compound 35 or 38. Label-free quantification (LFQ) protein mass spectrometry was used to identify compound-specific interactors. The SCC040 head and neck squamous cell carcinoma (HNSCC) cell line was chosen since SPIN1 expression in squamous cell carcinoma tumors was strongly correlated to smooth muscle actin and galectin1 , which may contribute to cell invasion and proliferation. ${ }^{32}$

Scheme 3. Synthesis of affinity probe 41 .

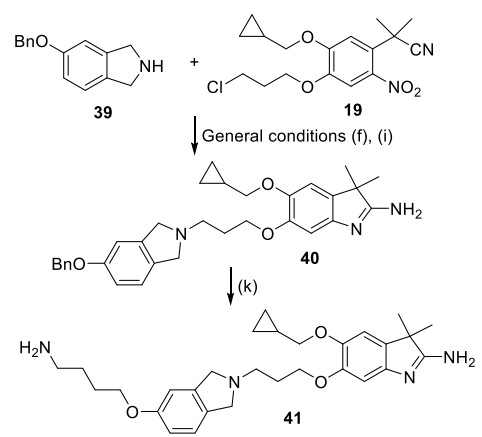

Conditions: general conditions (f) $2^{\circ}$ amine, $\mathrm{KI}, \mathrm{K}_{2} \mathrm{CO}_{3}, \mathrm{CH}_{3} \mathrm{CN}$, $80{ }^{\circ} \mathrm{C}, 48 \mathrm{~h}$; general conditions (i) $\mathrm{Zn}, \mathrm{AcOH}, 110{ }^{\circ} \mathrm{C}, 2 \mathrm{~h}, 63 \%$ over 2 steps; (k) Pd/C, $\mathrm{H}_{2}, \mathrm{MeOH}, \mathrm{rt}, 18$ h; 4-(Boc-amino)butyl bromide, $\mathrm{K}_{2} \mathrm{CO}_{3}$, DMF, rt, $48 \mathrm{~h}$; TFA, DCM, rt, $2 \mathrm{~h}, 12 \%$ overall. 
Gratifyingly, the only interacting partners of 35 were SPIN1, SPIN4 and the C11ORF84 (SPIN.DOC) protein (Figure 7, SI data.xls). The recent report by Bae et al. found that SPIN.DOC interacts with SPIN1 (as well as the other SPIN proteins) to mask Tudor domain 2 and impede its ability to bind to H3K4me3. ${ }^{22}$ However, our results indicate that the SPIN1 domain 2 is not impeded by the presence of SPIN.DOC, since both proteins were pulled down by the affinity probe $\mathbf{4 1}$, which interacts with Tudor domain 2. It is possible that the affinity probe $\mathbf{4 1}$ can bind to, and pull down, SPIN.DOC directly. However, since SPIN.DOC was shown to bind directly to all SPIN proteins, ${ }^{22}$ it is more likely that the affinity probe $\mathbf{4 1}$ can pulldown SPIN.DOC as a complex with the SPINs, and that compound $\mathbf{3 5}$ competes only with the interaction of SPIN1, or SPIN4, and the affinity probe. The results here seems to indicate that the interaction site of SPIN.DOC with the SPIN proteins is independent of Tudor domain 2, and therefore, further investigation is required.
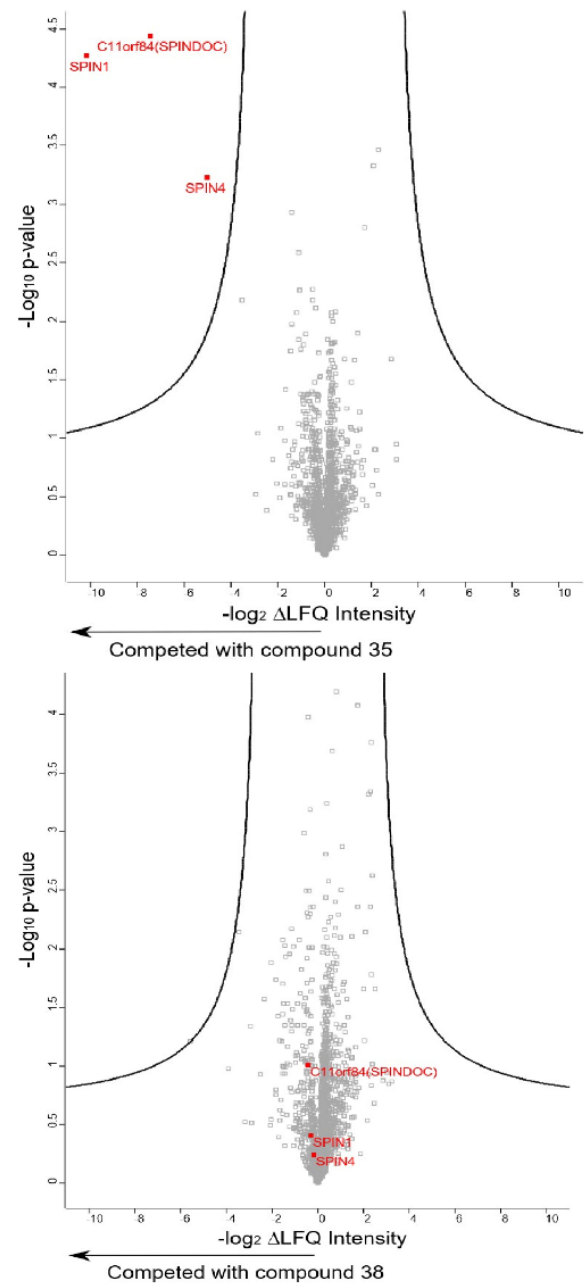

Figure 7. Chemoproteomic analyses using compound $\mathbf{3 5}$ (Top) or 38 (Bottom) as competitors in human squamous carcinoma cell line SCC040 lysates.

Taken together, all the selectivity data demonstrates that compound $\mathbf{3 5}$ has excellent in vitro specificity, including in a cellular context.

The proliferation, cell cycle and apoptosis of SCC040 cells was assessed following treatment with compound $\mathbf{3 5}$ and 38, (Figure S7). A moderate decrease in proliferation and increase in apoptosis was observed for compound $\mathbf{3 5}$, but not $\mathbf{3 8}$. Although this response was significant, it was not as dramatic as we expected. Therefore, to gain a greater understanding of role of SPIN1 in SCC040 cells, RNAseq analysis was performed (Figure S8-S11,
SI data.xls). RNA was isolated after treatment with compound $\mathbf{3 5}$ or 38 ( $1 \mu \mathrm{M}$ for 5 or 7 days), or with SPIN1 specific siRNA (2 or 4 days). ${ }^{7}$ Analysis of the induced changes identified 50 genes that were modulated by both compound $\mathbf{3 5}$ and the siRNA (Figure 8, SI data.xls). The overlap of genes modulated by compound $\mathbf{3 5}$ and 38 was minimal, which indicates that the transcriptional signature induced by compound $\mathbf{3 5}$ can be attributed to on-target effects.

When the transcriptional modulation induced by compound 35 is compared to that of siRNA SPIN1 knockdown, in many cases the genes appear to be modulated in opposing directions. However, since several genes were upregulated at the first timepoint, but were downregulated at the second (S100A8, S100A9, CLDN8, SLPI, S100P), it is clear that the transcriptional modulation is a dynamic process, and therefore, at a given timepoint the siRNA knockdown data may not be comparable to the compound inhibition data at that timepoint.

The identification of 6 members of the serine protease inhibitor superfamily (SERPINB4, SERPINB3, SERPINB13, SLPI, SPINK5, PI3) $)^{33-40}$ and 3 members of the S100 proteins (S100P, S100A8, S100A9), ${ }^{36,41-45}$ as well as, a chemokine (CCL5) and an immune checkpoint inhibitor (NT5E (CD73)), suggests that SPIN1 may have a role in the activation of inflammatory responses. The recent report by Bae et al. showed that treatment with the weak SPIN1 inhibitor EML631 $\left(\mathrm{K}_{\mathrm{D}} 4 \mu \mathrm{M}\right)$ resulted in the decreased expression of the pro-inflammatory cytokine IL1 $\beta,{ }^{46}$ as well as, BST2, which has been shown to activate the NF- $\mathrm{kB}$ inflammatory pathway in nasopharyngeal cancer. ${ }^{47}$ Inflammation within the tumor microenvironment (cancer-related inflammation (CRI)) is now known to be a significant contributing factor in the development and progression of cancer, particularly in tumor invasion and metastasis. ${ }^{44,48}$

Of the 50 genes identified here, a number have previously been reported to be differentially expressed (GBP2, ${ }^{49}$ DUOX2, ${ }^{50}$ IGFBP $3^{51-52}$ ), or were associated with a more invasive and metastatic phenotype (AKR1B10, ${ }^{53}$ FABP5, ${ }^{53-54}$ HSPB1, ${ }^{53}$ KRT1, ${ }^{53}$ S100A9, ${ }^{53}$ CNFN, ${ }^{55}$ ID2,${ }^{56}$ LCN2 (NGAL), ${ }^{57}$ IGFBP2, ${ }^{52}$ IFIT1 $1,{ }^{58}$ $\mathrm{CCL}^{48,59-60}$ ) in squamous cell carcinomas (SCC).

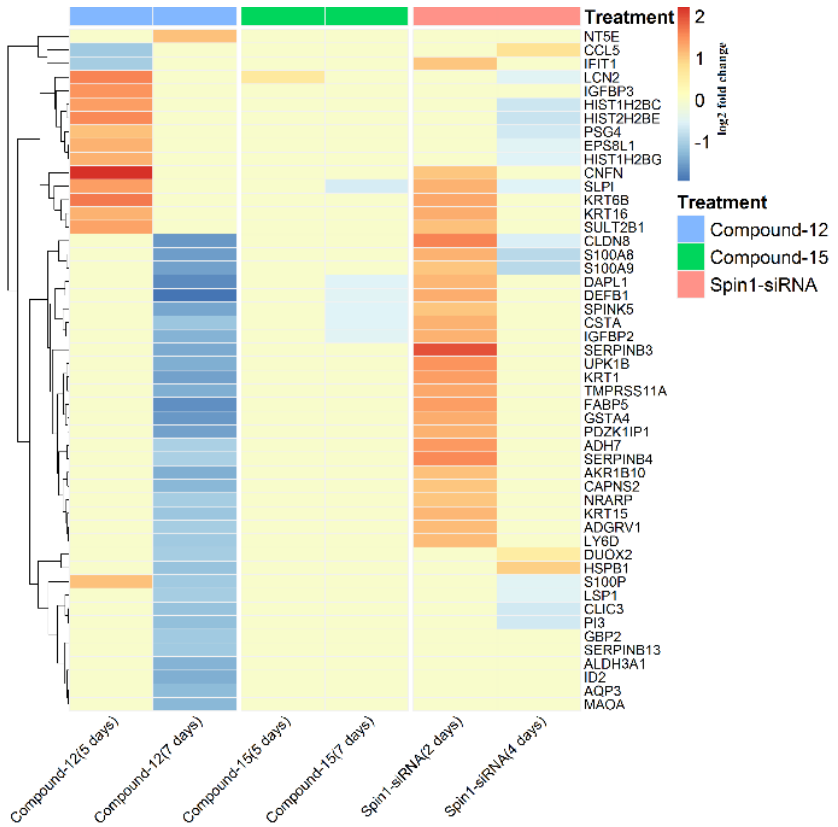

Figure 8. Heatmap of overlapping genes modulated by compound 35 and siRNA knockdown in SCC040 cells.

Importantly, compound $\mathbf{3 5}$ seems to modulate these genes in a direction which opposes that identified in these previously reported studies, thus may be of therapeutic value. 
In order to aid with the further development of compound $\mathbf{3 5}$ as a potential in vivo candidate, the in vitro permeability, stability and aqueous solubility were assessed. The intrinsic clearance, half-life and permeability were all within acceptable ranges, and the compound showed no efflux (Table 4). Compound $\mathbf{3 5}$ is highly soluble, with an aqueous solubility of $>100 \mu \mathrm{M}$ (SI, Table S11). Therefore, despite its large molecular weight, compound 35 is suitable for further development.

Table 4. In vitro permeability and stability of compound 35 performed by Cyprotex Ltd.

\begin{tabular}{|c|c|c|c|c|}
\hline \multicolumn{3}{|c|}{ Caco-2 Permeability } & \multicolumn{2}{|c|}{ Stability in Liver Microsomes } \\
\hline $\begin{array}{c}\text { AB P Papp } \\
\left(10^{-6} \mathrm{cms}^{-1}\right)\end{array}$ & $\underset{\left(10^{-6} \mathrm{cms}^{-1}\right)}{\text { BA Papp }}$ & $\begin{array}{l}\text { Efflux Ratio } \\
\text { (BA/AB) }\end{array}$ & $\begin{array}{c}\text { CLint } \\
(\mu \mathrm{L} / \mathrm{min} / \mathrm{mg} \\
\text { protein) }\end{array}$ & $\begin{array}{c}\mathbf{t}_{1 / 2} \\
(\mathrm{~min})\end{array}$ \\
\hline 6.59 & 6.07 & 0.92 & $\begin{array}{l}20.3^{\mathrm{a}} \\
37.4^{\mathrm{b}}\end{array}$ & $\begin{array}{l}68.4^{\mathrm{a}} \\
37.1^{\mathrm{b}}\end{array}$ \\
\hline
\end{tabular}

\section{DISCUSSION AND CONCLUSIONS}

We have developed a first-in-class potent, selective and cellactive chemical probe of the SPIN subfamily of Tudor domains. The selectivity of compound $\mathbf{3 5}$ and $\mathbf{3 8}$ has been extensively characterized and the value of this CPP as a tool to study the biology of SPIN proteins was demonstrated by analysis of the induced transcriptional changes. This analysis highlighted a significant number of genes that have previously been reported to be associated with CRI and/or cancer metastasis. Taken together, this data seems to suggest that SPIN1 may play a role in CRI and/or the metastatic potential of tumors, and warrants further investigation. The availability of a potent and cell active inhibitor, as well as, an inactive control compound, will facilitate and expedite the future evaluation of SPIN1. For future work we have named compound 35 VinSpinIn (Vinnie's Spindlin Inhibitor) and 38 VinSpinIC (Vinnie's Spindlin Inactive Control). For a limited period, this CPP, along with other highly characterized chemical probes, are freely available to the scientific community via online request from https://www.thesgc.org/chemical-probes.

\section{EXPERIMENTAL SECTION}

\section{Chemistry General Materials and Methods}

All solvents and reagents were purchased from commercial sources and used without further purification. Chemical reactions were carried out in anhydrous solvents, which were purchased from Acros Organics. Thin Layer Chromatography (TLC) was carried out using aluminum plates coated with 60 F254 silica gel and were visualized using UV light ( 254 or $365 \mathrm{~nm}$ ) unless otherwise stated. Automated flash chromatography was carried out on a Biotage Isolera One flash column chromatography system (LPLC), using Biotage ${ }^{\circledR}$ SNAP KP-Sil cartridges (unless otherwise state). All end compounds were purified to $\geq 95 \%$ purity, as indicated by analytical reverse phase high performance liquid chromatography (RP-HPLC) using UV and ELSD detection, as well as, ${ }^{1} \mathrm{H}$ NMR and ${ }^{13} \mathrm{C}$ NMR. Analytical HPLC and low resolution mass spectrometry was carried out on a waters LCMS system (Waters 2767 Sample Manager, Waters SFO System Fluidics Organizer, Waters 2545 Binary Gradient Module, 2 x Waters 515 HPLC pumps, Waters 2998 Photodiode Array Detector, Waters 2424 ELS Detector, Waters SQ Detector 2). Analytical separation was carried out on a Kinetex $5 \mu \mathrm{M}$ EVO C18 column $(100 \mathrm{~mm} \times$ $3.0 \mathrm{~mm}, 100 \mathrm{~A}$ ) using a flow rate of $2 \mathrm{~mL} / \mathrm{min}$ in a $3 \mathrm{~min}$ gradient elution. The mobile phase was a mixture of $93 \%$ water, $5 \%$ acetonitrile, and $2 \%$ of $0.5 \mathrm{M}$ ammonium acetate adjusted to $\mathrm{pH} 6$ with glacial acetic acid (solvent A) and 18\% water, $80 \%$ acetonitrile, and $2 \%$ of $0.5 \mathrm{M}$ ammonium acetate adjusted to $\mathrm{pH} 6$ with glacial acetic acid (solvent B). Gradient elution was as follows:
95:5 (A/B) $0.35 \mathrm{~min}, 95: 5(\mathrm{~A} / \mathrm{B})$ to $5: 95(\mathrm{~A} / \mathrm{B})$ over $1 \mathrm{~min}, 5: 95$ $(\mathrm{A} / \mathrm{B})$ over $0.75 \mathrm{~min}$, and then reversion back to $95: 5(\mathrm{~A} / \mathrm{B})$ over $0.1 \mathrm{~min}$ and $95: 5(\mathrm{~A} / \mathrm{B})$ over $0.8 \mathrm{~min}$.

NMR spectra were recorded using a Bruker Avance $400 \mathrm{MHz}$ spectrometer using the indicated deuterated solvent. Chemical shifts $(\delta)$ are quoted in parts per million $(\mathrm{ppm})$ and referenced to the residual solvent peak. Multiplicities are denoted as s- singlet, d- doublet, t- triplet and q- quartet, and derivatives thereof. Coupling constants are recorded in $\mathrm{Hz}$ and rounded to the nearest 0.1 Hz. Two-dimensional NMR experiments (COSY, HSQC) were used to aid the assignment of $1 \mathrm{H}$ and $13 \mathrm{C}$ spectra. Atom numbering in structures is purely for the purposes of assignment and does not reflect any numbering conventions.

All end compounds were examined to confirm that they did not contain known classes of pan-assay interference motifs.

Conditions (a); Synthesis of 1-(4-(benzyloxy)-3methoxyphenyl)cyclopentane-1-carbonitrile (2); The procedure of Barbasiewicz et al. was used with slight modification. ${ }^{61}$ Potassium hydroxide $(8.44 \mathrm{~g}, 150 \mathrm{mmol}, 12 \mathrm{eq})$ and water $(2.8 \mathrm{~mL})$ were added to a stirring solution of 2-(4-(benzyloxy)-3methoxyphenyl)acetonitrile 1 (3.17 g, $12.51 \mathrm{mmol}, 1 \mathrm{eq})$ and 1,4dibromobutane $(2.99 \mathrm{~mL}, 25.03 \mathrm{mmol}, 2.0 \mathrm{eq})$ in toluene $(100$ $\mathrm{mL}$ ). The mixture was cooled to $0{ }^{\circ} \mathrm{C}$ and tetrabutylammonium bromide $(60.5 \mathrm{mg}, 0.188 \mathrm{mmol}, 0.015 \mathrm{eq})$ was added. The mixture was stirred at $0{ }^{\circ} \mathrm{C}$ for approx. $15 \mathrm{~min}$, then heated to $80{ }^{\circ} \mathrm{C}$ with slow stirring $(50 \mathrm{rpm})$ until most of the $\mathrm{KOH}$ had dissolved (approx. $1 \mathrm{~h}$ ). The mixture was heated at reflux and stirred vigorously for a further $2 \mathrm{~h}$. Water $(75 \mathrm{~mL})$ was added to the cooled reaction mixture. The organic phase was separated, washed with water $(3 \times 50 \mathrm{~mL})$, dried $\left(\mathrm{Na}_{2} \mathrm{SO}_{4}\right)$, evaporated and the residue was purified by chromatography using a gradient of $0-40 \%$ of $[1: 1$ DCM:EtOAc] in cyclohexane to give compound 2 (3.545 g, 11.53 mmol, $92 \%)$ as a white solid. ${ }^{1} \mathrm{H}$ NMR $\left(400 \mathrm{MHz}, \mathrm{CDCl}_{3}\right) \delta 7.23-$ $7.16(\mathrm{~m}, 4 \mathrm{H}), 6.82(\mathrm{~s}, 2 \mathrm{H}), 4.13(\mathrm{t}, J=6.3 \mathrm{~Hz}, 2 \mathrm{H}), 3.96(\mathrm{~s}, 4 \mathrm{H})$, $3.81(\mathrm{~d}, J=6.9 \mathrm{~Hz}, 2 \mathrm{H}), 2.94(\mathrm{t}, J=7.4 \mathrm{~Hz}, 2 \mathrm{H}), 2.16-1.88(\mathrm{~m}$, $10 \mathrm{H}), 1.32-1.22(\mathrm{~m}, 1 \mathrm{H}), 0.62-0.56(\mathrm{~m}, 2 \mathrm{H}), 0.35-0.30(\mathrm{~m}, 2 \mathrm{H})$. ${ }^{13} \mathrm{C}$ NMR $\left(101 \mathrm{MHz}, \mathrm{CDCl}_{3}\right) \delta 149.74,147.76,136.95,132.76(4$ x C), 128.59, 127.91, $127.23(3 \mathrm{x} \mathrm{CH}), 124.58$ (C), 118.03, $113.89,110.28(3 \times \mathrm{CH}), 71.06\left(\mathrm{CH}_{2}\right), 56.18\left(\mathrm{CH}_{3}\right), 47.39(\mathrm{C})$, 40.37, $24.11\left(2 \times \mathrm{CH}_{2}\right)$. HPLC: $\mathrm{t}_{\mathrm{R}} 1.80 \mathrm{~min} . \mathrm{MS}(\mathrm{ESI}+): \mathrm{m} / \mathrm{z}$ $325.2\left[\mathrm{M}+\mathrm{NH}_{4}\right]^{+}$.

Conditions (b); Synthesis of 2-(4-(benzyloxy)-3methoxyphenyl)-2-methylpropanenitrile (3); A solution of the 2-(4-(benzyloxy)-3-methoxyphenyl)acetonitrile 1 (1.387 g, 5.48 mmol, $1 \mathrm{eq}$ ) and sodium hydroxide (aq. $50 \mathrm{wt} \%, 1.752 \mathrm{~g}, 21.90$ mmol, $4 \mathrm{eq})$ in DMSO $(10 \mathrm{~mL})$ were cooled to $0{ }^{\circ} \mathrm{C}$. Iodomethane $(1.36 \mathrm{ml}, 21.90 \mathrm{mmol}, 4 \mathrm{eq})$ was added dropwise and the mixture was stirred at room temperature for $1 \mathrm{~h}$ after the addition. The mixture was diluted with water $(100 \mathrm{~mL})$ and the product was extracted with toluene $(3 \times 30 \mathrm{~mL})$, dried $\left(\mathrm{Na}_{2} \mathrm{SO}_{4}\right)$ and evaporated to give compound $3(1.508 \mathrm{~g}, 5.36 \mathrm{mmol}, 98 \%)$ as a yellow oil. ${ }^{1} \mathrm{H}$ NMR (400 MHz, $\left.\mathrm{CDCl}_{3}\right) \delta$ 7.45-7.42 (m, 2H), 7.40-7.34 (m, 2H), 7.33-7.29 (m, 1H), $7.00(\mathrm{~d}, J=2.2 \mathrm{~Hz}, 1 \mathrm{H}), 6.92$ (dd, $J=$ 8.4, $2.2 \mathrm{~Hz}, 1 \mathrm{H}), 6.87(\mathrm{~d}, J=8.4 \mathrm{~Hz}, 1 \mathrm{H}), 5.15$ (s, 2H), 3.92 (s, $3 \mathrm{H}), 1.70(\mathrm{~s}, 6 \mathrm{H}) .{ }^{13} \mathrm{C} \mathrm{NMR}\left(101 \mathrm{MHz}, \mathrm{CDCl}_{3}\right) \delta 149.76,147.73$, 136.93, 134.49 ( 4 x C), 128.59, 127.92, 127.24 (3 x CH), 124.71 (C), 117.07, 113.94, 109.43 (3 x CH), $71.06\left(\mathrm{CH}_{2}\right), 56.19\left(\mathrm{CH}_{3}\right)$, $36.76(\mathrm{C}), 29.25\left(\mathrm{CH}_{3}\right)$. HPLC: $\mathrm{t}_{\mathrm{R}} 1.80 \mathrm{~min}$. MS $(\mathrm{ESI}+): \mathrm{m} / \mathrm{z}$ $299.6\left[\mathrm{M}+\mathrm{NH}_{4}\right]^{+}$.

General conditions (c); The procedure of Sweis et al. was used with slight modification. ${ }^{24}$ A solution of 2 or 3 (7.0 mmol, 1 eq), in 1:1 acetic acid:acetic anhydride $(5 \mathrm{~mL})$, was added dropwise to a solution of glacial acetic acid $(5 \mathrm{~mL})$, acetic anhydride $(5 \mathrm{~mL})$ and nitric acid $(70 \%, 5 \mathrm{~mL})$, maintaining the internal temperature of the reaction between 0 and $-5^{\circ} \mathrm{C}$. The mixture was 
stirred at $0{ }^{\circ} \mathrm{C}$ for approx. 30 min after the addition. The reaction mixture was poured over crushed ice and stirred vigorously for approx. 10 mins. The product was isolated by filtration and rinsed thoroughly with cold water.

1-(4-(benzyloxy)-5-methoxy-2-nitrophenyl)cyclopentane-1carbonitrile (4); Prepared using general conditions (c) from compound 2. (95\%) pale yellow solid. ${ }^{1} \mathrm{H}$ NMR (400 $\mathrm{MHz}$, $\left.\mathrm{CDCl}_{3}\right) \delta 7.50(\mathrm{~s}, 1 \mathrm{H}), 7.45-7.32(\mathrm{~m}, 5 \mathrm{H}), 6.99(\mathrm{~s}, 1 \mathrm{H}), 5.18(\mathrm{~s}$, $2 \mathrm{H}), 3.97$ (s, 3H), 2.75-2.64 (m, 2H), 2.10-1.98 (m, 4H), 1.98$1.81(\mathrm{~m}, 2 \mathrm{H}) .{ }^{13} \mathrm{C}$ NMR $\left(101 \mathrm{MHz}, \mathrm{CDCl}_{3}\right) \delta 152.62,147.46$, 142.50, 135.38 (4 x C), 128.83, 128.53, 127.54 (3 x CH), 127.49, $122.81(2 \times \mathrm{C}), 111.15,110.92(2 \times \mathrm{CH}), 71.37\left(\mathrm{CH}_{2}\right), 56.47$ (CH3), $45.43(\mathrm{C}), 38.95,23.84\left(2 \mathrm{x} \mathrm{CH}_{2}\right)$. HPLC: tR $1.87 \mathrm{~min} . \mathrm{MS}$ (ESI+): m/z $370.6\left[\mathrm{M}+\mathrm{NH}_{4}\right]^{+}$.

2-(4-(benzyloxy)-5-methoxy-2-nitrophenyl)-2methylpropanenitrile (5); Prepared using general conditions (c) from compound 3. (87\%) pale yellow solid. ${ }^{1} \mathrm{H}$ NMR $(400 \mathrm{MHz}$, $\left.\mathrm{CDCl}_{3}\right) \delta 7.45-7.31(\mathrm{~m}, 6 \mathrm{H}), 7.06(\mathrm{~s}, 1 \mathrm{H}), 5.17(\mathrm{~s}, 2 \mathrm{H}), 3.98(\mathrm{~s}$, $3 \mathrm{H}), 1.89(\mathrm{~s}, 6 \mathrm{H}) .{ }^{13} \mathrm{C} \mathrm{NMR}\left(101 \mathrm{MHz}, \mathrm{CDCl}_{3}\right) \delta 152.54,147.40$, 142.23, 135.36 (4 x C), 128.82, $128.52(2 \times \mathrm{CH}), 127.77$ (C), $127.53(\mathrm{CH}), 123.00(\mathrm{C}), 111.08,110.19(2 \times \mathrm{CH}), 71.39(\mathrm{CH} 2)$, $56.47\left(\mathrm{CH}_{3}\right), 36.44(\mathrm{C}), 28.36\left(\mathrm{CH}_{3}\right) . \mathrm{HPLC}: \mathrm{t}_{\mathrm{R}} 1.71 \mathrm{~min} . \mathrm{MS}$ $(\mathrm{ESI}+): \mathrm{m} / \mathrm{z} 327.2[\mathrm{M}+\mathrm{H}]^{+}$.

General Conditions (d); Palladium $(10 \%$ on carbon $(50 \%$ wet), 0.2 weight eq) was added to a solution of Benzyl aryl ether 4, 14 or 15 (2.0 mmol, 1 eq) in 9:1 EtOAc:EtOH $(50 \mathrm{~mL})$. The system was flushed with $\mathrm{N}_{2}$, followed by $\mathrm{H}_{2}$ and the mixture was stirred at room temperature under $\mathrm{H}_{2}$. The reaction was monitored closely by TLC and was complete in approximately $30 \mathrm{~min}$. The catalyst was removed by filtration through a bed of celite ${ }^{\circledR}$ and the solvent was evaporated. The residue was purified by automated flash chromatography using a gradient elution of $0-10 \% \mathrm{MeOH}$ in DCM.

1-(4-hydroxy-5-methoxy-2-nitrophenyl)cyclopentane-1carbonitrile (6); Prepared using general conditions (d) from benzyl aryl ether 4. (84\%) pale yellow oil. ${ }^{1} \mathrm{H}$ NMR $(400 \mathrm{MHz}$, $\left.\mathrm{CDCl}_{3}\right) \delta 7.46(\mathrm{~s}, 1 \mathrm{H}), 6.96(\mathrm{~s}, 1 \mathrm{H}), 5.86(\mathrm{bs}, 1 \mathrm{H}), 4.00(\mathrm{~s}, 3 \mathrm{H})$, 2.74-2.63 (m, 2H), 2.10-1.96 (m, 4H), 1.96-1.82 (m, 2H). ${ }^{13} \mathrm{C}$ NMR $\left(101 \mathrm{MHz}, \mathrm{CDCl}_{3}\right) \delta 149.34,145.26,143.38,126.20$, $122.88(5 \times \mathrm{C}), 112.56,109.99(2 \times \mathrm{CH}), 56.44\left(\mathrm{CH}_{3}\right), 45.38(\mathrm{C})$, 38.95, $23.80\left(2 \times \mathrm{CH}_{2}\right)$. HPLC: $\mathrm{t}_{\mathrm{R}} 1.41 \mathrm{~min}$. MS (ESI+): $\mathrm{m} / \mathrm{z}$ $263.2[\mathrm{M}+\mathrm{H}]^{+}$.

General conditions (e); Phenol 6, 12, 13, 16 or 17 (3.0 mmol, $1 \mathrm{eq})$, potassium carbonate ( $2 \mathrm{eq})$ and the required alkyl bromide (1.5 eq) (1-bromo-3-chloropropane for 7, 18, 19; (bromomethyl)cyclopropane for 14, 15; 1-bromo-4-chlorobutane for 20, 21) were heated in ACN $(20 \mathrm{~mL})$ at $80{ }^{\circ} \mathrm{C}$ overnight. The cooled reaction mixture was evaporated and the residue was purified by automated flash chromatography using a gradient elution of 50$100 \%$ DCM in cyclohexane.

\section{1-(4-(3-chloropropoxy)-5-methoxy-2-}

nitrophenyl)cyclopentane-1-carbonitrile (7); Prepared using general conditions (e) from phenol 6. (97\%) yellow solid. ${ }^{1} \mathrm{H}$ NMR (400 MHz, $\left.\mathrm{CDCl}_{3}\right) \delta 7.49(\mathrm{~s}, 1 \mathrm{H}), 7.00(\mathrm{~s}, 1 \mathrm{H}), 4.24(\mathrm{t}, J=$ $5.9 \mathrm{~Hz}, 2 \mathrm{H}), 3.97(\mathrm{~s}, 3 \mathrm{H}), 3.78(\mathrm{t}, J=6.2 \mathrm{~Hz}, 2 \mathrm{H}), 2.77-2.66(\mathrm{~m}$, $2 \mathrm{H}), 2.36-2.28(\mathrm{~m}, 2 \mathrm{H}), 2.12-1.98(\mathrm{~m}, 4 \mathrm{H}), 1.98-1.84(\mathrm{~m}, 2 \mathrm{H}) .{ }^{13} \mathrm{C}$ NMR $\left(101 \mathrm{MHz}, \mathrm{CDCl}_{3}\right) \delta 150.52,145.67,140.67,125.56$, $120.87(5 \times \mathrm{C}), 108.95,108.68(2 \times \mathrm{C}), 64.03\left(\mathrm{CH}_{2}\right), 54.49\left(\mathrm{CH}_{3}\right)$, 43.49 (C), 39.21, 37.04, 29.92, $21.92\left(4 \times \mathrm{CH}_{2}\right)$. HPLC: $\mathrm{t}_{\mathrm{R}} 1.73$ min. $\mathrm{MS}(\mathrm{ESI}+): \mathrm{m} / \mathrm{z} 339.2[\mathrm{M}+\mathrm{H}]^{+}$.

General conditions (f); Alkyl chloride 7 or 18-21 (0.25 mmol, $1 \mathrm{eq})$, potassium iodide ( $2 \mathrm{eq})$, potassium carbonate (3 eq) and the required secondary amine $(1.5 \mathrm{eq})$ were heated in $\mathrm{ACN}$ at $80{ }^{\circ} \mathrm{C}$ $(5 \mathrm{~mL})$ for $48 \mathrm{~h}$. The cooled reaction mixture was evaporated and the residue was purified by automated flash chromatography using a gradient elution of $0-5 \% \mathrm{MeOH}$ in $\mathrm{DCM}$.

\section{1-(5-methoxy-2-nitro-4-(3-(pyrrolidin-1-}

yl)propoxy)phenyl)cyclopentane-1-carbonitrile (8); Prepared using general conditions (f) from alkyl chloride 7. (91\%) yellow oil. ${ }^{1} \mathrm{H}$ NMR (400 MHz, $\left.\mathrm{CDCl}_{3}\right) \delta 7.50(\mathrm{~s}, 1 \mathrm{H}), 6.96(\mathrm{~s}, 1 \mathrm{H}), 4.15$ $(\mathrm{t}, J=6.6 \mathrm{~Hz}, 2 \mathrm{H}), 3.95(\mathrm{~s}, 3 \mathrm{H}), 2.75-2.65(\mathrm{~m}, 2 \mathrm{H}), 2.62(\mathrm{t}, J=$ $7.2 \mathrm{~Hz}, 2 \mathrm{H}), 2.55-2.48(\mathrm{~m}, 4 \mathrm{H}), 2.10-1.97(\mathrm{~m}, 6 \mathrm{H}), 1.97-1.83(\mathrm{~m}$, 2H), 1.83-1.74 (m, 4H). ${ }^{13} \mathrm{C}$ NMR $\left(101 \mathrm{MHz}, \mathrm{CDCl}_{3}\right) \delta 152.30$, 148.00, 142.69, 126.89, $122.87(5 \times \mathrm{C}), 110.82,110.39(2 \times \mathrm{CH})$, $68.03(\mathrm{CH} 2), 56.45\left(\mathrm{CH}_{3}\right), 54.22,52.65\left(2 \times \mathrm{CH}_{2}\right), 45.39(\mathrm{C})$, 38.94, 28.44, 23.83, $23.48\left(4 \mathrm{x} \mathrm{CH}_{2}\right)$. HPLC: $\mathrm{t}_{\mathrm{R}} 1.33 \mathrm{~min} . \mathrm{MS}$ (ESI+): m/z $374.4[\mathrm{M}+\mathrm{H}]^{+}$.

General conditions (g); Aspects of the procedures of Chae et $a l$. and Magano et al. were used with modifications. ${ }^{62-63}$ Dodecane-1-thiol (2.4 eq) was added to a solution of potassium tertbutoxide $(2.5 \mathrm{eq})$ in dry DMF $(40 \mathrm{~mL})$ under $\mathrm{N}_{2}$, which resulted in a heavy white precipitate. The mixture was cooled in an ice bath and a solution of methyl aryl ether 4,5 or $8(5.0 \mathrm{mmol}, 1 \mathrm{eq})$ in dry DMF $(20 \mathrm{~mL})$ was added dropwise maintaining the temperature at $0{ }^{\circ} \mathrm{C}$. After the addition, the mixture was heated to 50 ${ }^{\circ} \mathrm{C}$ for approx. 2 hours and monitored by TLC. Solid sodium hydrogen sulfate (4 eq) was added to the cooled reaction and the mixture was stirred vigorously until a color change, from orange to pale yellow, occurred. The solvent was evaporated and the residue was purified by automated flash chromatography using a gradient elution of $50-100 \%$ DCM in cyclohexane as eluent.

\section{1-(5-hydroxy-2-nitro-4-(3-(pyrrolidin-1-}

yl)propoxy)phenyl)cyclopentane-1-carbonitrile (9); Prepared using general conditions ( $g$ ) from methyl aryl ether 8, but was purified using a Biotage ${ }^{\circledR}$ SNAP KP-NH column, with a gradient elution of $0-10 \% \mathrm{MeOH}$ in DCM. (88\%) yellow oil. ${ }^{1} \mathrm{H}$ NMR $\left(400 \mathrm{MHz}, \mathrm{CDCl}_{3}\right) \delta 7.78(\mathrm{~s}, 1 \mathrm{H}), 6.81(\mathrm{~s}, 1 \mathrm{H}), 4.01(\mathrm{t}, J=5.0 \mathrm{~Hz}$, $2 \mathrm{H}), 3.10-2.98(\mathrm{~m}, 6 \mathrm{H}), 2.70-2.58(\mathrm{~m}, 2 \mathrm{H}), 2.11-1.89(\mathrm{~m}, 10 \mathrm{H})$, 1.89-1.74 (m, 2H). ${ }^{13} \mathrm{C}$ NMR (101 MHz, $\left.\mathrm{CDCl}_{3}\right) \delta 163.33$, 146.70, 135.96, 133.40, 123.53 ( $5 \times \mathrm{C}), 120.68,118.32(2 \times \mathrm{CH})$, 72.75, 54.55, 53.79 (3 x CH$), 45.55$ (C), 38.88, 26.65, 23.77, $23.21\left(4 \times \mathrm{CH}_{2}\right)$. HPLC: $t_{\mathrm{R}} 1.25 \mathrm{~min}$. MS (ESI+): $\mathrm{m} / \mathrm{z} 360.3$ $[\mathrm{M}+\mathrm{H}]^{+}$.

Conditions (h); Phenol 9 (0.25 mmol, 1 eq), potassium carbonate (2 eq) and (Bromomethyl)cyclopropane (1.5 eq) were heated in DMF $(5 \mathrm{~mL})$ at $50{ }^{\circ} \mathrm{C}$ overnight. The cooled reaction mixture was evaporated and the residue was purified by automated flash chromatography using a gradient elution of $0-5 \% \mathrm{MeOH}$ in DCM. The product was immediately subjected to general conditions (i).

General conditions (i); The procedure of Sweis et al. was used with modifications. ${ }^{24}$ Zinc powder $(10 \mathrm{eq})$ was added to the nitroarene $(0.25 \mathrm{mmol}, 1 \mathrm{eq})$ and the mixture was stirred vigorously in glacial acetic acid $(5 \mathrm{~mL})$ at $110^{\circ} \mathrm{C}$ for $2 \mathrm{~h}$. The acetic acid was evaporated and the residue was dissolved in DCM $(2 \mathrm{~mL})$. Solid potassium carbonate (10 eq) was added and the mixture was stirred vigorously until $\mathrm{CO}_{2}$ was no longer liberated. The mixture was directly loaded onto a Biotage ${ }^{\circledR}$ SNAP KP-NH column and the product was eluted with an eluent of $0-10 \% \mathrm{MeOH}$ in DCM.

5'-(cyclopropylmethoxy)-6'-(3-(pyrrolidin-1yl)propoxy)spiro[cyclopentane-1,3'-indol]-2'-amine (10); Prepared using general conditions (h) and (i) from phenol 9. (6\%) colorless oil that solidifies on standing. ${ }^{1} \mathrm{H}$ NMR $(400 \mathrm{MHz}$, $\left.\mathrm{CDCl}_{3}\right) \delta 6.81(\mathrm{~s}, 1 \mathrm{H}), 6.79(\mathrm{~s}, 1 \mathrm{H}), 4.05(\mathrm{t}, J=6.4 \mathrm{~Hz}, 2 \mathrm{H}), 3.79$ $(\mathrm{d}, J=7.0 \mathrm{~Hz}, 2 \mathrm{H}), 2.64(\mathrm{t}, J=7.6,2 \mathrm{H}), 2.55-2.47(\mathrm{~m}, 2 \mathrm{H}), 2.10-$ $1.88(\mathrm{~m}, 10 \mathrm{H}), 1.83-1.72(\mathrm{~m}, 4 \mathrm{H}), 1.31-1.19(\mathrm{~m}, 1 \mathrm{H}), 0.61-0.54$ $(\mathrm{m}, 2 \mathrm{H}), 0.33-0.27(\mathrm{~m}, 2 \mathrm{H}) .{ }^{13} \mathrm{C}$ NMR $\left(101 \mathrm{MHz}, \mathrm{CDCl}_{3}\right) \delta$ $179.50,150.08,149.18,144.05,135.05$ (5 x C) $, 112.13,103.44(2$ $\mathrm{x} \mathrm{CH}), 76.68,67.81\left(2 \times \mathrm{CH}_{2}\right), 58.62$ (C), 54.30, 53.36, 38.10, 
29.06, 26.61, $23.48\left(6 \times \mathrm{CH}_{2}\right), 10.86(\mathrm{C}), 3.13\left(\mathrm{CH}_{2}\right)$. HPLC: $100 \%$, tr $1.13 \mathrm{~min}$. MS (ESI+): $\mathrm{m} / \mathrm{z} 384.5[\mathrm{M}+\mathrm{H}]^{+}$.

\section{6'-(3-(isoindolin-2-yl)propoxy)-5'-}

methoxyspiro[cyclopentane-1,3'-indol]-2'-amine (11); Prepared using general conditions (f) and (i) from alkyl chloride 7. (29\%) colorless oil that solidifies on standing. ${ }^{1} \mathrm{H}$ NMR $(400 \mathrm{MHz}$, $\left.\mathrm{CDCl}_{3}\right) \delta 7.22-7.14(\mathrm{~m}, 5 \mathrm{H}), 6.84(\mathrm{~s}, 1 \mathrm{H}), 6.77(\mathrm{~s}, 1 \mathrm{H}), 4.15(\mathrm{t}, J$ $=6.6 \mathrm{~Hz}, 2 \mathrm{H}), 3.95(\mathrm{~s}, 4 \mathrm{H}), 3.85(\mathrm{~s}, 3 \mathrm{H}), 2.93(\mathrm{t}, J=7.3 \mathrm{~Hz}, 2 \mathrm{H})$, 2.17-1.90 (m, 10H). ${ }^{13} \mathrm{C}$ NMR $\left(101 \mathrm{MHz}, \mathrm{CDCl}_{3}\right) \delta 179.30$, 148.76, 148.25, 145.11, 140.17, 134.90 (6 x C), 126.64, 122.26, 107.72, $103.30(4 \times \mathrm{CH}), 67.61,59.20\left(\mathrm{CH}_{2}\right), 58.78(\mathrm{C}), 57.68$ $\left(\mathrm{CH}_{3}\right)$, 52.90, 38.08, 28.87, $26.69\left(\mathrm{CH}_{2}\right)$. HPLC: 96\%, tR 1.19 min. MS (ESI+): m/z $392.4[\mathrm{M}+\mathrm{H}]^{+}$.

1-(4-(benzyloxy)-5-hydroxy-2-nitrophenyl)cyclopentane-1carbonitrile (12); Prepared using general conditions (g) from methyl aryl ether 4. (86\%) yellow oil. ${ }^{1} \mathrm{H}$ NMR $(400 \mathrm{MHz}$, $\left.\mathrm{CDCl}_{3}\right) \delta 7.59(\mathrm{~s}, 1 \mathrm{H}), 7.47-7.38(\mathrm{~m}, 5 \mathrm{H}), 7.09(\mathrm{~s}, 1 \mathrm{H}), 6.09(\mathrm{~s}$, $1 \mathrm{H}), 5.18(\mathrm{~s}, 2 \mathrm{H}), 2.73-2.63(\mathrm{~m}, 2 \mathrm{H}), 2.09-1.97(\mathrm{~m}, 4 \mathrm{H}), 1.94-$ $1.81(\mathrm{~m}, 2 \mathrm{H}) .{ }^{13} \mathrm{C} \mathrm{NMR}\left(101 \mathrm{MHz}, \mathrm{CDCl}_{3}\right) \delta 149.53,144.63$, 142.14, 134.57 (4 x C), 129.11, 129.01 (2 x CH), 128.72 (C), $128.16(\mathrm{CH}), 122.66(\mathrm{C}), 114.47,110.30(2 \times \mathrm{CH}), 71.88\left(\mathrm{CH}_{2}\right)$, 44.97 (C), 39.03, $23.75\left(2 \times \mathrm{CH}_{2}\right)$. HPLC: $98 \%$, tR $1.64 \mathrm{~min}$. MS (ESI+): m/z $339.3[\mathrm{M}+\mathrm{H}]^{+}$.

2-(4-(benzyloxy)-5-hydroxy-2-nitrophenyl)-2-

methylpropanenitrile (13); Prepared using general conditions (g) from methyl aryl ether 5. (79\%) yellow oil. ${ }^{1} \mathrm{H}$ NMR $(400 \mathrm{MHz}$, $\left.\mathrm{CDCl}_{3}\right) \delta 7.51(\mathrm{~s}, 1 \mathrm{H}), 7.47-7.36(\mathrm{~m}, 5 \mathrm{H}), 7.12(\mathrm{~s}, 1 \mathrm{H}), 5.17(\mathrm{~s}$, $2 \mathrm{H}), 1.87$ (s, 6H). ${ }^{13} \mathrm{C}$ NMR $\left(101 \mathrm{MHz}, \mathrm{CDCl}_{3}\right) \delta 149.61,144.65$, $141.89,134.61,129.24$ (5 x C), 129.11, 129.01, 128.17 (3 x CH), $122.60(\mathrm{C}), 113.50,110.28(2 \times \mathrm{CH}), 71.87\left(\mathrm{CH}_{2}\right), 35.43(\mathrm{C})$, $28.83\left(\mathrm{CH}_{3}\right)$ HPLC: $\mathrm{t}_{\mathrm{R}} 1.63 \mathrm{~min} . \mathrm{MS} \quad(\mathrm{ESI}+): \mathrm{m} / \mathrm{z} 330.6$ $\left[\mathrm{M}+\mathrm{NH}_{4}\right]^{+}$

1-(4-(benzyloxy)-5-(cyclopropylmethoxy)-2-

nitrophenyl)cyclopentane-1-carbonitrile (14); Prepared using general conditions (e) from phenol 12. (99\%) yellow solid. ${ }^{1} \mathrm{H}$ NMR (400 MHz, $\left.\mathrm{CDCl}_{3}\right) \delta 7.51(\mathrm{~s}, 1 \mathrm{H}), 7.46-7.32(\mathrm{~m}, 5 \mathrm{H}), 6.99$ (s, 1H), 5.19 (s, 2H), $3.97(\mathrm{~d}, J=6.9 \mathrm{~Hz}, 2 \mathrm{H}), 2.73-2.61(\mathrm{~m}, 2 \mathrm{H})$, 2.10-1.96 (m, 4H), 1.96-1.81 (m, 2H), 1.37-1.23 (m, 1H), 0.71$0.67(\mathrm{~m}, 2 \mathrm{H}), 0.42-0.38(\mathrm{~m}, 2 \mathrm{H}) .{ }^{13} \mathrm{C}$ NMR $\left(101 \mathrm{MHz}, \mathrm{CDCl}_{3}\right) \delta$ 152.38, 147.88, 142.54, 135.72 (4 x C), 128.75, 128.37 ( $2 \times \mathrm{CH})$, $127.56(\mathrm{C}), 127.34(\mathrm{CH}), 122.85(\mathrm{C}), 113.23,112.04(2 \times \mathrm{CH})$, 74.57, 71.45 (2 x CH), 45.36 (C), 38.94, 23.83 (2 x CH 2$), 10.14$ $\left(\mathrm{CH}_{3}\right), 3.48\left(\mathrm{CH}_{2}\right)$. HPLC: $\mathrm{t}_{\mathrm{R}} 2.03 \mathrm{~min} . \mathrm{MS}(\mathrm{ESI}+): \mathrm{m} / \mathrm{z} 410.7$ $\left[\mathrm{M}+\mathrm{NH}_{4}\right]^{+}$.

2-(4-(benzyloxy)-5-(cyclopropylmethoxy)-2-nitrophenyl)-2methylpropanenitrile (15); Prepared using general conditions (e) from phenol 13. (92\%) yellow solid. ${ }^{1} \mathrm{H} \mathrm{NMR}\left(400 \mathrm{MHz}, \mathrm{CDCl}_{3}\right)$ $\delta 7.46-7.31(\mathrm{~m}, 6 \mathrm{H}), 7.07(\mathrm{~s}, 1 \mathrm{H}), 5.18(\mathrm{~s}, 2 \mathrm{H}), 3.98(\mathrm{~d}, J=6.9$ $\mathrm{Hz}, 2 \mathrm{H}), 1.87(\mathrm{~s}, 6 \mathrm{H}), 1.37-1.28(\mathrm{~m}, 1 \mathrm{H}), 0.71-0.66(\mathrm{~m}, 2 \mathrm{H}), 0.42-$ $0.378(\mathrm{~m}, 2 \mathrm{H}) .{ }^{13} \mathrm{C} \mathrm{NMR}\left(101 \mathrm{MHz}, \mathrm{CDCl}_{3}\right) \delta 152.30,147.81$, 142.26, 135.71 (4 x C), 128.73, $128.36(2 \times \mathrm{CH}), 127.86$ (C), $127.33(\mathrm{CH}), 123.04(\mathrm{C}), 112.46,111.98(2 \times \mathrm{CH}), 74.55,71.47$ $\left(2 \times \mathrm{CH}_{2}\right), 36.34(\mathrm{C}), 28.39\left(\mathrm{CH}_{3}\right), 10.12(\mathrm{CH}), 3.46\left(\mathrm{CH}_{2}\right)$. HPLC: $t_{R} 1.84 \mathrm{~min}$. MS (ESI+): $\mathrm{m} / \mathrm{z} 367.4[\mathrm{M}+\mathrm{H}]^{+}$.

1-(5-(cyclopropylmethoxy)-4-hydroxy-2-

nitrophenyl)cyclopentane-1-carbonitrile (16); Prepared using general conditions (d) from benzyl aryl ether 14. (76\%) pale yellow oil. ${ }^{1} \mathrm{H}$ NMR $\left(400 \mathrm{MHz}, \mathrm{CDCl}_{3}\right) \delta 7.47(\mathrm{~s}, 1 \mathrm{H}), 6.92(\mathrm{~s}, 1 \mathrm{H})$, $3.96(\mathrm{~d}, J=7.2 \mathrm{~Hz}, 2 \mathrm{H}), 2.72-2.59(\mathrm{~m}, 2 \mathrm{H}), 2.09-1.94(\mathrm{~m}, 4 \mathrm{H})$, 1.94-1.81 (m, 2H), 1.36-1.24 (m, 1H), 0.76-0.68 (m, 2H), 0.43$0.37(\mathrm{~m}, 2 \mathrm{H}) .{ }^{13} \mathrm{C} \mathrm{NMR}\left(101 \mathrm{MHz}, \mathrm{CDCl}_{3}\right) \delta 148.90,145.45$, 143.20, 126.11, 122.91 (5 x C), 112.51, 111.00 (2 x CH), 74.67 $\left(\mathrm{CH}_{2}\right), 45.36(\mathrm{C}), 38.95,23.78\left(2 \times \mathrm{CH}_{2}\right), 10.00(\mathrm{CH}), 3.54\left(\mathrm{CH}_{2}\right)$. HPLC: tR 1.67 min. MS (ESI+): m/z $320.5\left[\mathrm{M}+\mathrm{NH}_{4}\right]^{+}$. 1-(5-(cyclopropylmethoxy)-4-hydroxy-2-

nitrophenyl)cyclopentane-1-carbonitrile (17); Prepared using general conditions (d) from benzyl aryl ether 15. (95\%) pale yellow oil. ${ }^{1} \mathrm{H}$ NMR (400 MHz, $\left.\mathrm{CDCl}_{3}\right) \delta 7.36(\mathrm{~s}, 1 \mathrm{H}), 7.02(\mathrm{~s}, 1 \mathrm{H})$, $3.98(\mathrm{~d}, J=7.2 \mathrm{~Hz}, 2 \mathrm{H}), 1.86(\mathrm{~s}, 6 \mathrm{H}), 1.36-1.24(\mathrm{~m}, 1 \mathrm{H}), 0.74-$ $0.70(\mathrm{~m}, 2 \mathrm{H}), 0.42-0.39(\mathrm{~m}, 2 \mathrm{H}) .{ }^{13} \mathrm{C}$ NMR $\left(101 \mathrm{MHz}, \mathrm{CDCl}_{3}\right) \delta$ 152.20, 148.01, 142.60, 127.54, 122.82 (5 x C), 113.00, 111.38 (2 $\mathrm{x} \mathrm{CH}), 74.40,66.14\left(2 \times \mathrm{CH}_{2}\right), 45.34(\mathrm{C}), 41.16,38.96,31.92$, $23.82\left(4 \mathrm{x} \mathrm{CH}^{2}\right), 10.10(\mathrm{C}), 3.40\left(\mathrm{CH}^{2}\right)$. HPLC: $\mathrm{t}_{\mathrm{R}} 1.88 \mathrm{~min} . \mathrm{MS}$ (ESI+): m/z $379.3[\mathrm{M}+\mathrm{H}]^{+}$.

1-(4-(3-chloropropoxy)-5-(cyclopropylmethoxy)-2nitrophenyl)cyclopentane-1-carbonitrile (18); Prepared using general conditions (e) from phenol 16. (92\%) yellow solid. ${ }^{1} \mathrm{H}$ NMR (400 MHz, $\left.\mathrm{CDCl}_{3}\right) \delta 7.48(\mathrm{~s}, 1 \mathrm{H}), 6.97(\mathrm{~s}, 1 \mathrm{H}), 4.21(\mathrm{t}, J=$ $5.9 \mathrm{~Hz}, 2 \mathrm{H}), 3.93(\mathrm{~d}, J=6.9 \mathrm{~Hz}, 2 \mathrm{H}), 3.77(\mathrm{t}, J=6.2 \mathrm{~Hz}, 2 \mathrm{H})$, 2.74-2.62 (m, 2H), 2.33-2.27 (m, 2H), 2.10-1.96 (m, 4H), 1.95$1.82(\mathrm{~m}, 2 \mathrm{H}), 1.34-1.24(\mathrm{~m}, 1 \mathrm{H}), 0.70-0.66(\mathrm{~m}, 2 \mathrm{H}), 0.41-0.37(\mathrm{~m}$, $2 \mathrm{H}) .{ }^{13} \mathrm{C}$ NMR $\left(101 \mathrm{MHz}, \mathrm{CDCl}_{3}\right) \delta 152.20,148.01,142.60$, 127.54, $122.82(5 \times \mathrm{C}), 113.00,111.38(2 \times \mathrm{CH}), 74.40,66.14(2$ $\left.\mathrm{x} \mathrm{CH}_{2}\right), 45.34$ (C), 41.16, 38.96, 31.92, $23.82\left(4 \mathrm{x} \mathrm{CH}_{2}\right), 10.10$ (C), $3.40\left(\mathrm{CH}_{2}\right)$. HPLC: $t_{\mathrm{R}} 1.88 \mathrm{~min}$. MS (ESI+): $\mathrm{m} / \mathrm{z} 379.3$ $[\mathrm{M}+\mathrm{H}]^{+}$.

2-(4-(3-chloropropoxy)-5-(cyclopropylmethoxy)-2nitrophenyl)-2-methylpropanenitrile (19); Prepared using general conditions (e) from phenol 17. (99\%) yellow solid. ${ }^{1} \mathrm{H}$ NMR $\left(400 \mathrm{MHz}, \mathrm{CDCl}_{3}\right) \delta 7.37(\mathrm{~s}, 1 \mathrm{H}), 7.03(\mathrm{~s}, 1 \mathrm{H}), 4.20(\mathrm{t}, J=5.8$ $\mathrm{Hz}, 2 \mathrm{H}), 3.94(\mathrm{~d}, J=6.8 \mathrm{~Hz}, 2 \mathrm{H}), 3.77(\mathrm{t}, J=6.2 \mathrm{~Hz}, 2 \mathrm{H}), 2.32-$ $2.26(\mathrm{~m}, 2 \mathrm{H}), 1.86(\mathrm{~s}, 6 \mathrm{H}), 1.34-1.23(\mathrm{~m}, 1 \mathrm{H}), 0.69-0.65(\mathrm{~m}, 2 \mathrm{H})$, 0.40-0.36 (m, 2H). ${ }^{13} \mathrm{C}$ NMR (101 $\left.\mathrm{MHz}, \mathrm{CDCl}_{3}\right) \delta 152.15$, 147.94, 142.32, 127.85, 122.97 (5 x C), 112.18, $111.31(2 \times \mathrm{CH})$, 74.38, 66.15, 41.18 (3 x CH$), 36.20(\mathrm{C}), 31.92\left(\mathrm{CH}_{2}\right), 28.45$ $\left(\mathrm{CH}_{3}\right), 10.08(\mathrm{CH}), 3.38\left(\mathrm{CH}_{2}\right)$. HPLC: $\mathrm{t}_{\mathrm{R}} 1.88 \mathrm{~min}$. MS (ESI+): $\mathrm{m} / \mathrm{z} 370.6[\mathrm{M}+\mathrm{NH} 4]^{+}$.

1-(4-(4-chlorobutoxy)-5-(cyclopropylmethoxy)-2nitrophenyl)cyclopentane-1-carbonitrile (20); Prepared using general conditions (e) from phenol 16. (87\%) yellow solid. ${ }^{1} \mathrm{H}$ NMR $\left(400 \mathrm{MHz}, \mathrm{CDCl}_{3}\right) \delta 7.44(\mathrm{~s}, 1 \mathrm{H}), 6.96(\mathrm{~s}, 1 \mathrm{H}), 4.10(\mathrm{t}, J=$ $5.7 \mathrm{~Hz}, 2 \mathrm{H}), 3.93(\mathrm{~d}, J=6.9 \mathrm{~Hz}, 2 \mathrm{H}), 3.67(\mathrm{t}, J=6.2 \mathrm{~Hz}, 2 \mathrm{H})$, 2.72-2.64 (m, 2H), 2.09-1.96 (m, 8H), 1.96-1.79 (m, 2H), 1.36$1.25(\mathrm{~m}, 1 \mathrm{H}), 0.71-0.66(\mathrm{~m}, 2 \mathrm{H}), 0.41-0.37(\mathrm{~m}, 2 \mathrm{H}) .{ }^{13} \mathrm{C} \mathrm{NMR}$ $\left(101 \mathrm{MHz}, \mathrm{CDCl}_{3}\right) \delta 152.12,148.16,142.62,127.28,122.84(5 \mathrm{x}$ C), 112.93, $110.96(2 \times \mathrm{CH}), 74.43,68.95\left(2 \times \mathrm{CH}_{2}\right), 45.34(\mathrm{C})$, 44.59, 38.95, 29.41, 26.92, 26.31, $23.82\left(6 \times \mathrm{CH}_{2}\right), 10.11(\mathrm{CH})$, $3.39\left(\mathrm{CH}_{2}\right)$. HPLC: $\mathrm{t}_{\mathrm{R}} 1.92 \mathrm{~min}$. MS (ESI+): $\mathrm{m} / \mathrm{z} 393.4[\mathrm{M}+\mathrm{H}]^{+}$.

2-(4-(4-chlorobutoxy)-5-(cyclopropylmethoxy)-2nitrophenyl)-2-methylpropanenitrile (21); Prepared using general conditions (e) from phenol 17. (100\%) yellow solid. ${ }^{1} \mathrm{H}$ NMR $\left(400 \mathrm{MHz}, \mathrm{CDCl}_{3}\right) \delta 7.33(\mathrm{~s}, 1 \mathrm{H}), 7.03(\mathrm{~s}, 1 \mathrm{H}), 4.09(\mathrm{t}, J=5.8$ $\mathrm{Hz}, 2 \mathrm{H}), 3.94(\mathrm{~d}, J=6.9 \mathrm{~Hz}, 2 \mathrm{H}), 3.66(\mathrm{t}, J=6.3 \mathrm{~Hz}, 1 \mathrm{H}), 2.03-$ $1.98(\mathrm{~m}, 4 \mathrm{H}), 1.87(\mathrm{~s}, 6 \mathrm{H}), 1.35-1.25(\mathrm{~m}, 1 \mathrm{H}), 0.71-0.65(\mathrm{~m}, 2 \mathrm{H})$, 0.41-0.36 (m, 2H). ${ }^{13} \mathrm{C}$ NMR (101 MHz, $\left.\mathrm{CDCl}_{3}\right) \delta 152.04$, $148.09,142.33,127.57,123.02(5 \times \mathrm{C}), 112.14,110.89(2 \times \mathrm{CH})$, 74.41, 68.96, $44.60\left(3 \times \mathrm{CH}_{2}\right), 36.26(\mathrm{C}), 29.40\left(\mathrm{CH}_{2}\right), 28.43$ $\left(\mathrm{CH}_{3}\right), 26.30\left(\mathrm{CH}_{2}\right), 10.09(\mathrm{CH}), 3.38\left(\mathrm{CH}_{2}\right)$. HPLC: $\mathrm{t}_{\mathrm{R}} 1.93 \mathrm{~min}$. MS (ESI+): m/z $384.7[\mathrm{M}+\mathrm{H}]^{+}$.

5'-(cyclopropylmethoxy)-6'-(3-(5-methoxyisoindolin-2yl)propoxy)spiro[cyclopentane-1,3'-indol]-2'-amine (22); Prepared using general conditions (f) and (i) from alkyl chloride $\mathbf{1 8}$ (42\%) white solid. ${ }^{1} \mathrm{H}$ NMR $\left(400 \mathrm{MHz}, \mathrm{CDCl}_{3}\right) \delta 7.08(\mathrm{~d}, J=8.1$ $\mathrm{Hz}, 1 \mathrm{H}), 6.81(\mathrm{~s}, 1 \mathrm{H}), 6.80(\mathrm{~s}, 1 \mathrm{H}), 4.11(\mathrm{t}, J=6.4 \mathrm{~Hz}, 2 \mathrm{H}), 3.92$ (s, 2H), $3.89(\mathrm{~s}, 2 \mathrm{H}), 3.80(\mathrm{~d}, J=6.9 \mathrm{~Hz}, 2 \mathrm{H}), 3.78(\mathrm{~s}, 3 \mathrm{H}), 2.92$ (t, $J=7.3 \mathrm{~Hz}, 2 \mathrm{H}), 2.12-1.90(\mathrm{~m}, 10 \mathrm{H}), 1.31-1.21(\mathrm{~m}, 1 \mathrm{H}), 0.60-$ $0.56(\mathrm{~m}, 2 \mathrm{H}), 0.33-0.30(\mathrm{~m}, 2 \mathrm{H}) .{ }^{13} \mathrm{C}$ NMR $\left(101 \mathrm{MHz}, \mathrm{CDCl}_{3}\right) \delta$ 179.61, 158.96, 150.02, 148.75, 144.07, 141.62, 134.90, 132.20 (8 x C), 122.86, 112.51, 112.04, 107.97, 103.30 (5 x CH), 76.63, 67.54, 59.40, $58.59\left(4 \times \mathrm{CH}_{2}\right), 58.58(\mathrm{C}), 55.47\left(\mathrm{CH}_{3}\right)$, 53.01, 
38.16, 28.98, $26.60\left(4 \times \mathrm{CH}_{2}\right), 10.87(\mathrm{CH}), 3.16\left(\mathrm{CH}_{2}\right)$. HPLC: $98 \%$, tR $1.39 \mathrm{~min}$. MS (ESI+): $\mathrm{m} / \mathrm{z} 462.8[\mathrm{M}+\mathrm{H}]^{+}$.

\section{5'-(cyclopropylmethoxy)-6'-(3-(isoindolin-2-}

yl)propoxy)spiro[cyclopentane-1,3'-indol]-2'-amine (23); Prepared using general conditions (f) and (i) from alkyl chloride $\mathbf{1 8}$. (45\%) white solid. ${ }^{1} \mathrm{H}$ NMR $\left(400 \mathrm{MHz}, \mathrm{CDCl}_{3}\right) \delta$ 7.23-7.16 (m, $4 \mathrm{H}), 6.82(\mathrm{~s}, 2 \mathrm{H}), 4.13(\mathrm{t}, J=6.3 \mathrm{~Hz}, 2 \mathrm{H}), 3.96(\mathrm{~s}, 4 \mathrm{H}), 3.81(\mathrm{~d}, J$ $=6.9 \mathrm{~Hz}, 2 \mathrm{H}), 2.94(\mathrm{t}, J=7.4 \mathrm{~Hz}, 2 \mathrm{H}), 2.16-1.88(\mathrm{~m}, 10 \mathrm{H}), 1.32$ $1.22(\mathrm{~m}, 1 \mathrm{H}), 0.62-0.56(\mathrm{~m}, 2 \mathrm{H}), 0.35-0.30(\mathrm{~m}, 2 \mathrm{H}) .{ }^{13} \mathrm{C} \mathrm{NMR}$ $\left(101 \mathrm{MHz}, \mathrm{CDCl}_{3}\right) \delta 179.36,150.04,149.09,144.16,140.16$, 135.14 (6 x C), 126.65, 122.27, 112.02, 103.56 (4 x CH), 76.64, 67.55, $59.21\left(3 \mathrm{x} \mathrm{CH}_{2}\right), 58.64(\mathrm{C}), 52.99,38.09,29.00,26.62(4 \mathrm{x}$ $\left.\mathrm{CH}_{2}\right), 10.87(\mathrm{CH}), 3.16\left(\mathrm{CH}_{2}\right)$. HPLC: $100 \%$, tR $1.40 \mathrm{~min} . \mathrm{MS}$ (ESI+): $\mathrm{m} / \mathrm{z} 432.7[\mathrm{M}+\mathrm{H}]^{+}$.

5'-(cyclopropylmethoxy)-6'-(4-(isoindolin-2-

yl)butoxy)spiro[cyclopentane-1,3'-indol]-2'-amine (24); Prepared using general conditions (f) and (i) from alkyl chloride $\mathbf{2 0}$. (44\%) white solid. ${ }^{1} \mathrm{H}$ NMR $\left(400 \mathrm{MHz}, \mathrm{CDCl}_{3}\right) \delta$ 7.21-7.16 (m, $4 \mathrm{H}), 6.82(\mathrm{~s}, 1 \mathrm{H}), 6.80(\mathrm{~s}, 1 \mathrm{H}), 4.05(\mathrm{t}, J=6.4 \mathrm{~Hz}, 2 \mathrm{H}), 3.93(\mathrm{~s}$, $4 \mathrm{H}), 3.81(\mathrm{~d}, J=7.0 \mathrm{~Hz}, 2 \mathrm{H}), 2.79(\mathrm{t}, J=7.3 \mathrm{~Hz}, 2 \mathrm{H}), 2.10-1.88$ $(\mathrm{m}, 10 \mathrm{H}), 1.82-1.74(\mathrm{~m}, 2 \mathrm{H}), 1.33-1.22(\mathrm{~m}, 1 \mathrm{H}), 0.61-0.54(\mathrm{~m}$, 2H), 0.35-0.30 (m, 2H). ${ }^{13} \mathrm{C}$ NMR (101 MHz, $\left.\mathrm{CDCl}_{3}\right) \delta 179.49$, $150.10,149.06,144.09,140.21,140.21,134.97$ (7 x C), 126.62, $122.25,112.08,103.36$ ( 4 x CH), 76.63, 69.07, $59.11\left(3 \times \mathrm{CH}_{2}\right)$, 58.64 (C), 55.84, 38.11, 27.28, 26.61, 25.61 (5 x $\left.\mathrm{CH}_{2}\right), 10.88$ $(\mathrm{CH}), 3.16\left(\mathrm{CH}_{2}\right)$. HPLC: $100 \%, \mathrm{t}_{\mathrm{R}} 1.40 \mathrm{~min} . \mathrm{MS}(\mathrm{ESI}+): \mathrm{m} / \mathrm{z}$ $446.7[\mathrm{M}+\mathrm{H}]^{+}$.

5-(cyclopropylmethoxy)-6-(3-(isoindolin-2-yl)propoxy)-3,3dimethyl-3H-indol-2-amine (25); Prepared using general conditions (f) and (i) from alkyl chloride 19. (42\%) white solid. ${ }^{1} \mathrm{H}$ NMR (400 MHz, $\left.\mathrm{CDCl}_{3}\right) \delta$ 7.22-7.16 (m, $\left.4 \mathrm{H}\right), 6.84(\mathrm{~s}, 1 \mathrm{H}), 6.79$ $(\mathrm{s}, 1 \mathrm{H}), 4.13(\mathrm{t}, J=6.3 \mathrm{~Hz}, 2 \mathrm{H}), 3.96(\mathrm{~s}, 4 \mathrm{H}), 3.82(\mathrm{~d}, J=6.9 \mathrm{~Hz}$, $2 \mathrm{H}), 2.95(\mathrm{t}, J=7.4,2 \mathrm{H}), 2.15-2.07(\mathrm{~m}, 2 \mathrm{H}), 1.33-1.23(\mathrm{~m}, 7 \mathrm{H})$, 0.63-0.55 (m, 2H), 0.36-0.29 (m, 2H). ${ }^{13} \mathrm{C}$ NMR $(101 \mathrm{MHz}$, $\left.\mathrm{CDCl}_{3}\right) \delta 179.11,150.11,147.80,144.45,140.14,133.12(6 \times \mathrm{C})$, 126.66, 122.27, 111.42, $103.69(4 \times \mathrm{CH}), 76.31,67.61,59.20$, $52.96\left(4 \times \mathrm{CH}_{2}\right), 48.73(\mathrm{C}), 28.96\left(\mathrm{CH}_{2}\right), 25.08\left(\mathrm{CH}_{3}\right), 10.81$ $(\mathrm{CH}), 3.16\left(\mathrm{CH}_{2}\right)$. HPLC: $99 \%, \mathrm{t}_{\mathrm{R}} 1.34 \mathrm{~min}$. MS (ESI+): $\mathrm{m} / \mathrm{z}$ $406.7[\mathrm{M}+\mathrm{H}]^{+}$.

5-(cyclopropylmethoxy)-6-(4-(isoindolin-2-yl)butoxy)-3,3dimethyl-3H-indol-2-amine (26); Prepared using general conditions (f) and (i) from alkyl chloride 21. (39\%) white solid. ${ }^{1} \mathrm{H}$ NMR (400 MHz, $\left.\mathrm{CDCl}_{3}\right) \delta$ 7.21-7.16 (m, 4H), $6.83(\mathrm{~s}, 1 \mathrm{H}), 6.79$ $(\mathrm{s}, 1 \mathrm{H}), 4.06(\mathrm{t}, J=6.4 \mathrm{~Hz}, 2 \mathrm{H}), 3.93(\mathrm{~s}, 4 \mathrm{H}), 3.82(\mathrm{~d}, J=6.9 \mathrm{~Hz}$, 2H), $2.79(\mathrm{t}, J=7.4 \mathrm{~Hz}, 2 \mathrm{H}), 1.98-1.87(\mathrm{~m}, 2 \mathrm{H}), 1.84-1.75(\mathrm{~m}$, $2 \mathrm{H}), 1.32-1.21(\mathrm{~m}, 7 \mathrm{H}), 0.61-0.54(\mathrm{~m}, 2 \mathrm{H}), 0.37-0.28(\mathrm{~m}, 2 \mathrm{H}) .{ }^{13} \mathrm{C}$ NMR $\left(101 \mathrm{MHz}, \mathrm{CDCl}_{3}\right) \delta 179.22,150.13,148.57,144.25$, $140.19,133.28$ (6 x C), 126.61, 122.24, 111.47, 103.68 (4 x CH), 76.33, 69.10, 59.10, $55.84\left(4 \times \mathrm{CH}_{2}\right), 48.77$ (C), 27.27, 25.59 (2 x $\left.\mathrm{CH}_{2}\right), 25.09\left(\mathrm{CH}_{3}\right), 10.83(\mathrm{CH}), 3.16\left(\mathrm{CH}_{2}\right)$. HPLC: 95\%, tR 1.34 $\min$. MS (ESI+): $\mathrm{m} / \mathrm{z} 420.8[\mathrm{M}+\mathrm{H}]^{+}$.

5-(prop-2-yn-1-yloxy)isoindoline (27); Propargyl bromide (80 $\mathrm{wt} \%$ in toluene, $0.30 \mathrm{ml}, 2.81 \mathrm{mmol}, 1.1 \mathrm{eq}$ ) was added to a stirring mixture of tert-butyl 5-hydroxyisoindoline-2-carboxylate $(0.600 \mathrm{~g}, 2.55 \mathrm{mmol}, 1 \mathrm{eq})$ and potassium carbonate $(0.705 \mathrm{~g}$, $5.10 \mathrm{mmol}, 2 \mathrm{eq})$ in $\mathrm{ACN}(20 \mathrm{~mL})$, and the mixture was stirred at $40{ }^{\circ} \mathrm{C}$ overnight. The solvent was evaporated and the residue was purified by automated flash chromatography using a gradient elution of $0-5 \% \mathrm{MeOH}$ in DCM (and using Seebach's TLC stain to visualize the product) to give a colorless oil $(0.630 \mathrm{~g}, 2.30$ mmol, 90\%). The oil was dissolved in dry DCM $(20 \mathrm{~mL})$ and trifluoroacetic acid (TFA, $2 \mathrm{~mL}$ ) was added. The solution was stirred at room temperature for approximately $2 \mathrm{~h}$. The solvent was evaporated and the residue was purified by automated flash chromatography using a gradient elution of $0-10 \%$ of $\left[7 \mathrm{~N} \mathrm{NH}_{3}\right.$ in
$\mathrm{MeOH}]$ in DCM (and using ninhydrin TLC stain to visualize the product) to give compound $27(0.413 \mathrm{~g}, 2.38 \mathrm{mmol}, 93 \%(84 \%$ overall) $)$ as a brown solid. ${ }^{1} \mathrm{H}$ NMR $\left(400 \mathrm{MHz}, \mathrm{CDCl}_{3}\right) \delta 7.16(\mathrm{~d}$, $J=8.2 \mathrm{~Hz}, 1 \mathrm{H}), 6.90-6.82(\mathrm{~m}, 2 \mathrm{H}), 4.69$ (d, $J=2.4 \mathrm{~Hz}, 2 \mathrm{H}), 4.23$ $(\mathrm{s}, 2 \mathrm{H}), 4.20(\mathrm{~s}, 2 \mathrm{H}), 2.51(\mathrm{t}, J=2.4 \mathrm{~Hz}, 1 \mathrm{H}) .{ }^{13} \mathrm{C}$ NMR $(101$ $\left.\mathrm{MHz}, \mathrm{CDCl}_{3}\right) \delta 157.02,143.17,134.49$ (3 x C), 122.90, 113.85, $109.01(3 \times \mathrm{CH}), 78.66(\mathrm{C}), 75.45(\mathrm{CH}), 56.12,53.04,52.29(3 \mathrm{x}$ $\mathrm{CH}_{2}$ ). HPLC: $\mathrm{t}_{\mathrm{R}} 0.71 \mathrm{~min}$. MS (ESI+): $\mathrm{m} / \mathrm{z} 174.2[\mathrm{M}+\mathrm{H}]^{+}$.

5-(but-3-yn-1-yloxy)isoindoline (28); But-3-yn-1-ol (0.39 ml, $5.10 \mathrm{mmol}, 1.2 \mathrm{eq})$ was added to a solution of tert-butyl 5hydroxyisoindoline-2-carboxylate (1.000 g, $4.25 \mathrm{mmol}, 1 \mathrm{eq})$ and triphenylphosphine $(1.115 \mathrm{~g}, 4.25 \mathrm{mmol}, 1 \mathrm{eq})$ in dry THF (20 $\mathrm{mL}$ ) under $\mathrm{N}_{2}$. The solution was cooled to $0{ }^{\circ} \mathrm{C}$ and diethyl azodicarboxylate (DEAD, $0.669 \mathrm{ml}, 4.25 \mathrm{mmol}, 1 \mathrm{eq})$ was added. The solution was heated at $60{ }^{\circ} \mathrm{C}$ for $5 \mathrm{~h}$. The solvent was evaporated and the residue was purified by automated flash chromatography using a gradient elution of $50-100 \%$ DCM in cyclohexane (and using Seebach's TLC stain to visualize the product) to a colorless oil $(0.509 \mathrm{~g}, 1.77 \mathrm{mmol}, 42 \%)$. The oil was dissolved in dry DCM $(20 \mathrm{~mL})$ and trifluoroacetic acid (TFA, $2 \mathrm{~mL}$ ) was added. The solution was stirred at room temperature for approximately $2 \mathrm{~h}$. The solvent was evaporated and the residue was purified by automated flash chromatography using a gradient elution of $0-10 \%$ of $\left[7 \mathrm{~N} \mathrm{NH}_{3}\right.$ in $\mathrm{MeOH}$ ] in DCM (and using ninhydrin TLC stain to visualize the product) to give compound $28(0.302 \mathrm{~g}, 1.61$ mmol, $91 \%$ (38\% overall)) as a brown solid. ${ }^{1} \mathrm{H}$ NMR $(400 \mathrm{MHz}$, $\left.\mathrm{CDCl}_{3}\right) \delta 7.13(\mathrm{~d}, J=8.2 \mathrm{~Hz}, 1 \mathrm{H}), 6.83-6.72(\mathrm{~m}, 2 \mathrm{H}), 4.20(\mathrm{~s}$, 2H), 4.18 (s, 2H), 4.08 (t, $J=7.0 \mathrm{~Hz}, 2 \mathrm{H}), 2.67$ (td, $J=7.0,2.7$ $\mathrm{Hz}, 2 \mathrm{H}), 2.04(\mathrm{t}, J=2.7 \mathrm{~Hz}, 1 \mathrm{H}) .{ }^{13} \mathrm{C} \mathrm{NMR}\left(101 \mathrm{MHz}, \mathrm{CDCl}_{3}\right) \delta$ $157.87,143.29,134.07(3 \times \mathrm{C}), 122.90,113.57,108.70(3 \times \mathrm{CH})$, $80.46(\mathrm{C}), 69.87(\mathrm{CH}), 66.33,53.06,52.31,19.56\left(4 \times \mathrm{CH}_{2}\right)$. HPLC: tR $1.00 \mathrm{~min}$. MS (ESI+): $\mathrm{m} / \mathrm{z} 188.2[\mathrm{M}+\mathrm{H}]^{+}$.

2-(5-(cyclopropylmethoxy)-2-nitro-4-(3-(5-(prop-2-yn-1yloxy)isoindolin-2-yl)propoxy)phenyl)-2-methylpropanenitrile (29); Prepared using general conditions (f) from alkyl chloride 19 and alkyne functionalized isoindoline 27. (96\%) brown oil. ${ }^{1} \mathrm{H}$ NMR $\left(400 \mathrm{MHz}, \mathrm{CDCl}_{3}\right) \delta 7.37(\mathrm{~s}, 1 \mathrm{H}), 7.12(\mathrm{~d}, J=8.0 \mathrm{~Hz}, 1 \mathrm{H})$, $7.04(\mathrm{~s}, 1 \mathrm{H}), 6.85-6.80(\mathrm{~m}, 2 \mathrm{H}), 4.67(\mathrm{~d}, J=2.4 \mathrm{~Hz}, 2 \mathrm{H}), 4.19(\mathrm{t}$, $J=6.4 \mathrm{~Hz}, 2 \mathrm{H}), 4.00-3.90(\mathrm{~m}, 6 \mathrm{H}), 2.95(\mathrm{t}, J=6.9 \mathrm{~Hz}, 2 \mathrm{H}), 2.50$ $(\mathrm{t}, J=2.4 \mathrm{~Hz}, 1 \mathrm{H}), 2.19-2.09(\mathrm{~m}, 2 \mathrm{H}), 1.87(\mathrm{~s}, 6 \mathrm{H}), 1.36-1.25(\mathrm{~m}$, 1H), 0.74-0.62 (m, 2H), 0.46-0.34 (m, 2H). ${ }^{13} \mathrm{C} \mathrm{NMR} \mathrm{(101} \mathrm{MHz,}$ $\left.\mathrm{CDCl}_{3}\right) \delta 157.05,151.91,148.33,142.46,141.03,132.57,127.30$, 123.09 ( $8 \times \mathrm{C}), 123.00,113.85,112.48,110.96,109.25(5 \times \mathrm{CH})$, $78.62(\mathrm{C}), 75.46(\mathrm{CH}), 74.47,67.66,59.26,58.51,56.13,52.46(6$ x $\left.\mathrm{CH}_{2}\right), 36.34(\mathrm{C}), 28.39\left(\mathrm{CH}_{3}\right), 28.21\left(\mathrm{CH}_{2}\right), 10.14(\mathrm{CH}), 3.42$ $\left(\mathrm{CH}_{2}\right)$. HPLC: $\mathrm{t}_{\mathrm{R}} 1.75 \mathrm{~min}$. MS (ESI +$): \mathrm{m} / \mathrm{z} 490.4[\mathrm{M}+\mathrm{H}]^{+}$.

\section{2-(4-(3-(5-(but-3-yn-1-yloxy)isoindolin-2-yl)propoxy)-5-} (cyclopropylmethoxy)-2-nitrophenyl)-2-methylpropanenitrile (30); Prepared using general conditions (f) from alkyl chloride 19 and alkyne functionalized isoindoline 28. (98\%) brown oil. ${ }^{1} \mathrm{H}$ $\operatorname{NMR}\left(400 \mathrm{MHz}, \mathrm{CDCl}_{3}\right) \delta 7.38(\mathrm{~s}, 1 \mathrm{H}), 7.10(\mathrm{~d}, J=8.0 \mathrm{~Hz}, 1 \mathrm{H})$, $7.03(\mathrm{~s}, 1 \mathrm{H}), 6.79-6.74(\mathrm{~m}, 2 \mathrm{H}), 4.19(\mathrm{t}, J=6.4 \mathrm{~Hz}, 2 \mathrm{H}), 4.07(\mathrm{t}, J$ $=7.1 \mathrm{~Hz}, 2 \mathrm{H}), 3.98-3.90(\mathrm{~m}, 6 \mathrm{H}), 2.94(\mathrm{t}, J=7.0 \mathrm{~Hz}, 2 \mathrm{H}), 2.66$ $(\mathrm{td}, J=7.0,2.7 \mathrm{~Hz}, 2 \mathrm{H}), 2.17-2.11(\mathrm{~m}, 2 \mathrm{H}), 2.03(\mathrm{t}, J=2.7 \mathrm{~Hz}$, $1 \mathrm{H}), 1.87(\mathrm{~s}, 6 \mathrm{H}), 1.36-1.25(\mathrm{~m}, 1 \mathrm{H}), 0.70-0.65(\mathrm{~m}, 2 \mathrm{H}), 0.42-$ $0.38(\mathrm{~m}, 2 \mathrm{H}) .{ }^{13} \mathrm{C}$ NMR $\left(101 \mathrm{MHz}, \mathrm{CDCl}_{3}\right) \delta 157.92,151.92$, $148.33,142.45,141.10,132.11,127.30,123.08$ (7 x C), 123.01, 113.61, 112.46, 110.96, $108.97(5 \times \mathrm{CH}), 80.44(\mathrm{C}), 74.47\left(\mathrm{CH}_{2}\right)$, $69.87(\mathrm{CH}), 67.67,66.35,59.26,58.51,52.47\left(5 \times \mathrm{CH}_{2}\right), 36.30$ (C), $28.40\left(\mathrm{CH}_{3}\right), 28.21,19.54\left(\mathrm{CH}_{2}\right), 10.14(\mathrm{CH}), 3.42\left(\mathrm{CH}_{2}\right)$. HPLC: tR $1.77 \mathrm{~min}$. MS (ESI+): m/z $504.4[\mathrm{M}+\mathrm{H}]^{+}$.

2-(5-(cyclopropylmethoxy)-2-nitro-4-(4-(5-(prop-2-yn-1yloxy)isoindolin-2-yl)butoxy)phenyl)-2-methylpropanenitrile (31); Prepared using general conditions (f) from alkyl chloride 21 and alkyne functionalized isoindoline 27. (52\%) brown oil. ${ }^{1} \mathrm{H}$ NMR (400 MHz, $\left.\mathrm{CDCl}_{3}\right) \delta 7.34(\mathrm{~s}, 1 \mathrm{H}), 7.11(\mathrm{~d}, J=8.0 \mathrm{~Hz}, 1 \mathrm{H})$, 
7.03 (s, 1H), 6.86-6.78 (m, 2H), 4.67 (d, $J=2.4 \mathrm{~Hz}, 2 \mathrm{H}), 410$ (t, $J$ $=6.4 \mathrm{~Hz}, 2 \mathrm{H}), 3.98-3.85(\mathrm{~m}, 6 \mathrm{H}), 2.81(\mathrm{t}, J=7.2 \mathrm{~Hz}, 2 \mathrm{H}), 2.50$ $(\mathrm{t}, J=2.4 \mathrm{~Hz}, 1 \mathrm{H}), 2.0-1.94(\mathrm{~m}, 2 \mathrm{H}), 1.87(\mathrm{~s}, 6 \mathrm{H}), 1.82-1.74(\mathrm{~m}$, $2 \mathrm{H}), 1.36-1.26(\mathrm{~m}, 1 \mathrm{H}), 0.70-0.63(\mathrm{~m}, 2 \mathrm{H}), 0.42-0.38(\mathrm{~m}, 2 \mathrm{H}) .{ }^{13} \mathrm{C}$ NMR $\left(101 \mathrm{MHz}, \mathrm{CDCl}_{3}\right) \delta 156.94,151.94,148.31,142.40$, 141.40, 132.95, 127.23, 123.08 (8 x C), 122.95, 113.66, 112.30, 110.66, 109.23 (5 x CH), $78.95(\mathrm{C}), 75.44(\mathrm{CH}), 74.44,69.36$, 59.20, 58.44, 56.11, $55.59\left(6 \times \mathrm{CH}_{2}\right), 36.25(\mathrm{C}), 28.44\left(\mathrm{CH}_{3}\right)$, 26.70, $25.21\left(2 \times \mathrm{CH}_{2}\right), 10.15(\mathrm{CH}), 3.44\left(\mathrm{CH}_{2}\right)$. HPLC: $\mathrm{t}_{\mathrm{R}} 1.75$ min. MS (ESI+): m/z $504.4[\mathrm{M}+\mathrm{H}]^{+}$.

2-azido-1-(4-(2-(pyrrolidin-1-yl)ethyl)piperidin-1-yl)ethan1-one (32); 2-Azidoacetic acid $(0.23 \mathrm{ml}, 3.03 \mathrm{mmol}, 1.3 \mathrm{eq})$ was added to a stirring solution of $p$-toluenesulfonyl chloride $(0.489 \mathrm{~g}$, $2.57 \mathrm{mmol}, 1.1 \mathrm{eq}$ ) and diisopropylaminomethyl-polystyrene resin $(3 \mathrm{mmol} / \mathrm{g}, 2.333 \mathrm{~g}, 3 \mathrm{eq})$ in dry DCM $(20 \mathrm{~mL})$, and the mixture was stirred a room temperature for $1 \mathrm{~h}$. 4-(2-(Pyrrolidin-1yl)ethyl)piperidine ( $0.425 \mathrm{~g}, 2.33 \mathrm{mmol}, 1 \mathrm{eq})$ was added and the mixture was stirred for a further $1 \mathrm{~h}$. The mixture was filtered, and the filtrate was washed with $\mathrm{Na}_{2} \mathrm{CO}_{3}(1 \mathrm{M}, 50 \mathrm{~mL})$, dried $\left(\mathrm{Na}_{2} \mathrm{SO}_{4}\right)$ and evaporated to give compound $32(0.5217 \mathrm{~g}, 1.966$ mmol, $84 \%$ ) as a yellow solid. ${ }^{1} \mathrm{H}$ NMR $\left(400 \mathrm{MHz}, \mathrm{CDCl}_{3}\right) \delta 4.55$ (d, $J=13.3 \mathrm{~Hz}, 1 \mathrm{H}), 3.92$ (s, 1H), 3.61 (d, $J=13.5 \mathrm{~Hz}, 1 \mathrm{H}), 3.02$ $(\mathrm{td}, J=13.0,2.8 \mathrm{~Hz}, 0 \mathrm{H}), 2.62(\mathrm{td}, J=12.9,2.9 \mathrm{~Hz}, 1 \mathrm{H}), 2.55-$ $2.40(\mathrm{~m}, 6 \mathrm{H}), 1.84-1.71(\mathrm{~m}, 6 \mathrm{H}), 1.65-1.45(\mathrm{~m}, 3 \mathrm{H}), 1.22-1.08(\mathrm{~m}$, $2 \mathrm{H}) .{ }^{13} \mathrm{C} \mathrm{NMR}\left(101 \mathrm{MHz}, \mathrm{CDCl}_{3}\right) \delta 165.27(\mathrm{C}), 54.26,53.77$, 50.78, 45.39, 42.49, $35.36\left(6 \times \mathrm{CH}_{2}\right), 34.29(\mathrm{CH}), 32.75,31.82$, $23.40\left(3 \times \mathrm{CH}_{2}\right)$. HPLC: $\mathrm{t}_{\mathrm{R}} 0.67 \mathrm{~min}$. MS (ESI+): $\mathrm{m} / \mathrm{z} 266.3$ $[\mathrm{M}+\mathrm{H}]^{+}$.

3-azido-1-(4-(2-(pyrrolidin-1-yl)ethyl)piperidin-1-

yl)propan-1-one (33); 3-Azidopropanoic acid $(0.24 \mathrm{~mL}, 2.60$ mmol, 1.3 eq) was added to a stirring solution of $p$ toluenesulfonyl chloride $(0.418 \mathrm{~g}, 2.20 \mathrm{mmol}, 1.1 \mathrm{eq})$ and resinbound diisopropylaminomethyl-polystyrene $(3 \mathrm{mmol} / \mathrm{g}, 2.000 \mathrm{~g} 3$ eq) in dry DCM $(20 \mathrm{~mL})$, and the mixture was stirred a room temperature for 1 h. 4-(2-(Pyrrolidin-1-yl)ethyl)piperidine $(0.365$ $\mathrm{g}, 2.00 \mathrm{mmol}, 1 \mathrm{eq}$ ) was added and the mixture was stirred for a further $1 \mathrm{~h}$. The mixture was filtered, and the filtrate was washed with $\mathrm{Na}_{2} \mathrm{CO}_{3}(1 \mathrm{M}, 50 \mathrm{~mL})$, dried $\left(\mathrm{Na}_{2} \mathrm{SO}_{4}\right)$ and evaporated to give crude compound $\mathbf{3 3}$ (89\% but with a purity of approx. $90 \%$ ) as a yellow solid. Compound $\mathbf{3 3}$ was unstable and following the workup, approximately $10 \%$ of an alkene by-product had formed (presumably caused by elimination of the azide during evaporation) and therefore, was not characterized, but was used immediately as a crude mixture in the proceeding click reaction.

General conditions (j); Copper powder $(<425 \mu \mathrm{m}, 25 \mathrm{eq})$, azide 32 or 33 (2 eq) and alkyne 29, 30 or $31(0.20 \mathrm{mmol}, 1 \mathrm{eq})$ were stirred vigorously in DMF $(5 \mathrm{~mL})$ at $60{ }^{\circ} \mathrm{C}$, under $\mathrm{N}_{2}$, until all the alkyne starting material had been consumed (approx. $2 \mathrm{~h}$ ). The cooled reaction mixture was evaporated and the residue was purified by automated flash chromatography using a gradient elution of $0-10 \%$ of $\left[7 \mathrm{~N} \mathrm{NH}_{3}\right.$ in $\left.\mathrm{MeOH}\right]$ in DCM.

2-(4-((2-(3-((2-amino-5-(cyclopropylmethoxy)-3,3dimethyl-3H-indol-6-yl)oxy)propyl)isoindolin-5yl)oxy)methyl)-1H-1,2,3-triazol-1-yl)-1-(4-(2-(pyrrolidin-1yl)ethyl)piperidin-1-yl)ethan-1-one (34); Prepared using general conditions (j) and (i) from alkyne 29 and azide 32. (17\%) white solid. ${ }^{1} \mathrm{H}$ NMR $\left(400 \mathrm{MHz}, \mathrm{CDCl}_{3}\right) \delta 7.78(\mathrm{~s}, 1 \mathrm{H}), 7.08(\mathrm{~d}, J=8.1$ $\mathrm{Hz}, 1 \mathrm{H}), 6.85-6.76(\mathrm{~m}, 4 \mathrm{H}), 5.26-5.16(\mathrm{~m}, 4 \mathrm{H}), 4.51$ (d, $J=13.4$ $\mathrm{Hz}, 1 \mathrm{H}), 4.11(\mathrm{t}, J=6.2 \mathrm{~Hz}, 2 \mathrm{H}), 3.91-3.78(\mathrm{~m}, 7 \mathrm{H}), 3.11$ (t, $J=$ $6.5 \mathrm{~Hz}, 1 \mathrm{H}), 2.91(\mathrm{t}, J=7.3 \mathrm{~Hz}, 2 \mathrm{H}), 2.64(\mathrm{t}, J=13.0 \mathrm{~Hz}, 1 \mathrm{H})$, 2.55-2.45 (m, 6H), 2.11-2.05 (m, 2H), 1.84-1.72 (m, 6H), 1.68$1.54(\mathrm{~m}, 1 \mathrm{H}), 1.54-1.45(\mathrm{~m}, 2 \mathrm{H}), 1.36-1.21(\mathrm{~m}, 7 \mathrm{H}), 1.21-1.08(\mathrm{~m}$, 2H), 0.64-0.56 (m, 2H), 0.34-0.29 (m, 2H). ${ }^{13} \mathrm{C}$ NMR (101 MHz, $\left.\mathrm{CDCl}_{3}\right) \delta 179.37,162.92,157.57,150.37,147.91,144.89,144.55$, 141.67, 133.39, 132.81 (10 x C), 124.43, 122.97, 113.57, 111.46, 109.08, 102.28 (6 x CH), 76.23, 67.58, 62.32, 59.34, 58.55, 54.15,
53.64, 52.84, $51.05\left(9 \times \mathrm{CH}_{2}\right), 48.26(\mathrm{C}), 45.67,42.75,35.03(3 \mathrm{x}$ $\left.\mathrm{CH}_{2}\right), 34.17(\mathrm{CH}), 32.62,31.68,28.85\left(3 \times \mathrm{CH}_{2}\right), 25.09\left(\mathrm{CH}_{3}\right)$, $23.39\left(\mathrm{CH}_{2}\right), 10.75(\mathrm{CH}), 3.17(\mathrm{CH} 2)$. HPLC: 98\%, th $1.20 \mathrm{~min}$. MS (ESI+): m/z 726.0 $[\mathrm{M}+\mathrm{H}]^{+}, 363.8[\mathrm{M}+2 \mathrm{H}]^{2+}$.

2-(4-(2-((2-(3-((2-amino-5-(cyclopropylmethoxy)-3,3dimethyl-3H-indol-6-yl)oxy)propyl)isoindolin-5-yl)oxy)ethyl)1H-1,2,3-triazol-1-yl)-1-(4-(2-(pyrrolidin-1-yl)ethyl)piperidin1-yl)ethan-1-one (35); Prepared using general conditions (j) and (i) from alkyne 30 and azide $32 .(47 \%)$ white solid. ${ }^{1} \mathrm{H}$ NMR $(400$ $\left.\mathrm{MHz} \mathrm{CDCl}_{3}\right) \delta 7.62(\mathrm{~s}, 1 \mathrm{H}), 7.06(\mathrm{~d}, J=6.5 \mathrm{~Hz}, 1 \mathrm{H}), 6.82(\mathrm{~s}$, $1 \mathrm{H}), 6.78-6.70(\mathrm{~m}, 3 \mathrm{H}), 5.24-5.14(\mathrm{~m}, 2 \mathrm{H}), 4.51(\mathrm{~d}, J=13.4 \mathrm{~Hz}$, $1 \mathrm{H}), 4.22(\mathrm{t}, J=6.5 \mathrm{~Hz}, 2 \mathrm{H}), 4.11(\mathrm{t}, J=6.3 \mathrm{~Hz}, 2 \mathrm{H}), 3.93-3.76$ $(\mathrm{m}, 7 \mathrm{H}), 3.21(\mathrm{t}, J=6.5 \mathrm{~Hz}, 2 \mathrm{H}), 3.09(\mathrm{td}, J=13.2,2.5 \mathrm{~Hz}, 1 \mathrm{H})$, $2.91(\mathrm{t}, J=7.3 \mathrm{~Hz}, 2 \mathrm{H}), 2.63(\mathrm{td}, J=12.9,2.6 \mathrm{~Hz}, 1 \mathrm{H}), 2.53-2.41$ $(\mathrm{m}, 6 \mathrm{H}), 2.13-2.03(\mathrm{~m}, 2 \mathrm{H}), 1.84-1.70(\mathrm{~m}, 6 \mathrm{H}), 1.66-1.53(\mathrm{~m}$, 1H), 1.52-1.42 (m, 2H), 1.35-1.21 (m, 7H), 1.19-1.04 (m, 2H), 0.62-0.54 (m, 2H), 0.36-0.27 (m, 2H). ${ }^{13} \mathrm{C}$ NMR (101 MHz, $\left.\mathrm{CDCl}_{3}\right) \delta 179.3,163.2,158.0,150.0,148.3,144.9,144.2,141.7$, $133.3,132.5,(10 \times \mathrm{C}), 123.5,122.9,113.2,111.4,108.8,103.6(6$ $\mathrm{x} \mathrm{CH}), 76.3,67.6,67.0,59.4,58.6,54.3,53.7,53.0,51.1(9 \mathrm{x}$ $\left.\mathrm{CH}_{2}\right), 48.7(\mathrm{C}), 45.7,42.7,35.3\left(3 \mathrm{x} \mathrm{CH}_{2}\right), 34.2(\mathrm{CH}), 32.6,31.7$, 29.0, 26.4 (4 x CH 2$), 25.1\left(\mathrm{CH}_{3}\right), 23.4\left(\mathrm{CH}_{2}\right), 10.8(\mathrm{CH}), 3.2$ $\left(\mathrm{CH}_{2}\right)$. HPLC: $100 \%$, tR $1.21 \mathrm{~min}$. MS (ESI+): m/z $740.1[\mathrm{M}+\mathrm{H}]^{+}$, $370.9[\mathrm{M}+2 \mathrm{H}]^{2+}$.

\section{3-(4-(((2-(3-((2-amino-5-(cyclopropylmethoxy)-3,3-}

\section{dimethyl-3H-indol-6-yl)oxy)propyl)isoindolin-5-}

yl)oxy)methyl)-1H-1,2,3-triazol-1-yl)-1-(4-(2-(pyrrolidin-1yl)ethyl)piperidin-1-yl)propan-1-one (36); Prepared using general conditions (j) and (i) from alkyne 29 and azide 33. (31\%) while solid. ${ }^{1} \mathrm{H}$ NMR $\left(400 \mathrm{MHz}, \mathrm{CDCl}_{3}\right) \delta 7.76(\mathrm{~s}, 1 \mathrm{H}), 7.08(\mathrm{~d}, J$ $=8.2 \mathrm{~Hz}, 1 \mathrm{H}), 6.86-6.75(\mathrm{~m}, 4 \mathrm{H}), 5.15(\mathrm{~s}, 2 \mathrm{H}), 4.70(\mathrm{t}, J=6.3 \mathrm{~Hz}$, $2 \mathrm{H}), 4.51(\mathrm{~d}, J=13.3 \mathrm{~Hz}, 2 \mathrm{H}), 4.11(\mathrm{t}, J=6.3 \mathrm{~Hz}, 2 \mathrm{H}), 3.91(\mathrm{~s}$, 2H), 3.88 (s, 2H), $3.81(\mathrm{~d}, J=6.9 \mathrm{~Hz}, 2 \mathrm{H}), 3.71(\mathrm{~d}, J=13.4 \mathrm{~Hz}$, $2 \mathrm{H}), 2.97-2.87(\mathrm{~m}, 5 \mathrm{H}), 2.58-2.41(\mathrm{~m}, 7 \mathrm{H}), 2.13-2.03(\mathrm{~m}, 2 \mathrm{H})$, $1.82-1.66(\mathrm{~m}, 6 \mathrm{H}), 1.60-1.42(\mathrm{~m}, 3 \mathrm{H}), 1.35-1.22(\mathrm{~m}, 7 \mathrm{H}), 1.14-$ 0.98 (m, 2H), 0.61-0.54 (m, 2H), 0.34-0.28 (m, 2H). ${ }^{13} \mathrm{C}$ NMR $\left(101 \mathrm{MHz} \mathrm{CDCl}_{3}\right) \delta 179.26,167.35,157.60,150.05,148.39$, $144.28,143.84,141.70,133.31,132.82$ (10 x C), 124.25, 122.94, 113.49, 111.43, 108.99, $103.75(6 \times \mathrm{CH}), 76.32,67.57,62.24$, 59.37, 58.57, 54.24, 53.79, $52.97\left(8 \times \mathrm{CH}_{2}\right), 48.73(\mathrm{C}), 46.16$, 45.58, 42.16, $35.35\left(4 \times \mathrm{CH}_{2}\right), 34.32(\mathrm{CH}), 33.56,32.65,31.86$, $28.96\left(4 \times \mathrm{CH}_{2}\right), 25.07\left(\mathrm{CH}_{3}\right), 23.39\left(\mathrm{CH}_{2}\right), 10.82(\mathrm{CH}), 3.16$ $\left(\mathrm{CH}_{2}\right)$. HPLC: $99 \%, \mathrm{t}_{\mathrm{R}} 1.32 \mathrm{~min}$. MS (ESI+): m/z $740.1[\mathrm{M}+\mathrm{H}]^{+}$, $370.9[\mathrm{M}+2 \mathrm{H}]^{2+}$.

3-(4-(2-((2-(3-((2-amino-5-(cyclopropylmethoxy)-3,3dimethyl-3H-indol-6-yl)oxy)propyl)isoindolin-5-yl)oxy)ethyl)1H-1,2,3-triazol-1-yl)-1-(4-(2-(pyrrolidin-1-yl)ethyl)piperidin1-yl)propan-1-one (37); Prepared using general conditions (j) and (i) from alkyne 30 and azide 33. (14\%) while solid. ${ }^{1} \mathrm{H}$ NMR $\left(400 \mathrm{MHz} \mathrm{CDCl}_{3}\right) \delta 7.58(\mathrm{~s}, 1 \mathrm{H}), 7.07(\mathrm{~d}, J=8.2 \mathrm{~Hz}, 1 \mathrm{H}), 6.83$ $(\mathrm{s}, 1 \mathrm{H}), 7.06-6.83(\mathrm{~m}, 3 \mathrm{H}), 4.67(\mathrm{t}, J=6.5 \mathrm{~Hz}, 2 \mathrm{H}), 4.52(\mathrm{~d}, J=$ $12.9 \mathrm{~Hz}, 1 \mathrm{H}), 4.20(\mathrm{t}, J=6.6 \mathrm{~Hz}, 2 \mathrm{H}), 4.12(\mathrm{t}, J=6.3 \mathrm{~Hz}, 2 \mathrm{H})$, $3.91(\mathrm{~s}, 2 \mathrm{H}), 3.88(\mathrm{~s}, 2 \mathrm{H}), 3.81(\mathrm{~d}, J=7.0 \mathrm{~Hz}, 2 \mathrm{H}), 3.72(\mathrm{~d}, J=$ $14.0 \mathrm{~Hz}, 1 \mathrm{H}), 3.17(\mathrm{t}, J=6.6 \mathrm{~Hz}, 2 \mathrm{H}), 2.97-2.88(\mathrm{~m}, 5 \mathrm{H}), 2.56-$ $2.44(\mathrm{~m}, 7 \mathrm{H}), 2.12-2.05(\mathrm{~m}, 2 \mathrm{H}), 1.84-1.66(\mathrm{~m}, 6 \mathrm{H}), 1.60-1.43(\mathrm{~m}$, $3 \mathrm{H}), 1.35-1.20(\mathrm{~m}, 7 \mathrm{H}), 1.11-0.98(\mathrm{~m}, 2 \mathrm{H}), 0.62-0.54(\mathrm{~m}, 2 \mathrm{H})$, 0.36-0.27 (m, 2H). ${ }^{13} \mathrm{C}$ NMR (101 MHz, $\left.\mathrm{CDCl}_{3}\right) \delta 179.10$, $167.51,158.00,150.09,147.97,144.41,144.28,141.67,133.19$, $132.48(10 \times \mathrm{C}), 123.29,122.92,113.28,111.41,108.78,103.73$ (6 x CH), 76.31, 67.59, 67.10, 59.37, 58.59, 54.22, 53.77, $52.98(8$ $\mathrm{x} \mathrm{CH} 2), 48.72(\mathrm{C}), 46.02,45.58,42.13,35.18\left(4 \mathrm{x} \mathrm{CH}_{2}\right), 34.18$ $(\mathrm{CH}), 33.58,32.64,31.86,28.94,26.17\left(5 \times \mathrm{CH}_{2}\right), 25.07\left(\mathrm{CH}_{3}\right)$, $23.39\left(\mathrm{CH}_{2}\right), 10.81(\mathrm{CH}), 3.16\left(\mathrm{CH}_{2}\right)$. HPLC: $98 \%$, t $1.21 \mathrm{~min}$. MS (ESI+): m/z 754.1 [M+H] $]^{+}, 377.9[\mathrm{M}+2 \mathrm{H}]^{2+}$.

2-(4-(((2-(4-((2-amino-5-(cyclopropylmethoxy)-3,3dimethyl-3H-indol-6-yl)oxy)butyl)isoindolin-5-yl)oxy)methyl)- 
1H-1,2,3-triazol-1-yl)-1-(4-(2-(pyrrolidin-1-yl)ethyl)piperidin1-yl)ethan-1-one (38); Prepared using general conditions (j) and (i) from alkyne 31 and azide 32. (54\%) while solid. ${ }^{1} \mathrm{H}$ NMR (400 $\left.\mathrm{MHz} \mathrm{CDCl}_{3}\right) \delta 7.78(\mathrm{~s}, 1 \mathrm{H}), 7.07(\mathrm{~d}, J=8.0 \mathrm{~Hz}, 1 \mathrm{H}), 6.83-6.76$ $(\mathrm{m}, 4 \mathrm{H}), 5.26-5.16(\mathrm{~m}, 4 \mathrm{H}), 4.51(\mathrm{~d}, J=13.4 \mathrm{~Hz}, 2 \mathrm{H}), 4.05(\mathrm{t}, J=$ $6.3 \mathrm{~Hz}, 2 \mathrm{H}), 3.91-3.77(\mathrm{~m}, 7 \mathrm{H}) 3.11$ (td, $J=13.0,2.8 \mathrm{~Hz}, 1 \mathrm{H})$, $2.76(\mathrm{t}, J=7.3 \mathrm{~Hz}, 2 \mathrm{H}), 2.65(\mathrm{td}, J=13.0,3.0 \mathrm{~Hz}, 1 \mathrm{H}), 2.56-2.43$ $(\mathrm{m}, 6 \mathrm{H}), 1.96-1.86(\mathrm{~m}, 2 \mathrm{H}), 1.86-1.70(\mathrm{~m}, 8 \mathrm{H}), 1.67-1.54(\mathrm{~m}$, $1 \mathrm{H}), 1.54-1.45(\mathrm{~m}, 2 \mathrm{H}), 1.36-1.22(\mathrm{~m}, 7 \mathrm{H}), 1.22-1.07(\mathrm{~m}, 2 \mathrm{H})$, 0.62-0.54 (m, 2H), 0.36-0.27 (m, 2H). ${ }^{13} \mathrm{C}$ NMR (101 MHz, $\left.\mathrm{CDCl}_{3}\right) \delta 179.20,162.93,157.53,150.11,147.92,144.50,144.33$, $141.72,133.03,132.87$ (10 x C), 124.46, 122.93, 113.46, 111.42, $109.09,103.51(6 \times \mathrm{CH}), 76.28,69.09,62.29,59.24,58.48,55.83$, 54.24, 53.70, $51.05\left(9 \times \mathrm{CH}_{2}\right), 48.72(\mathrm{C}), 45.66,42.76,35.19(3 \mathrm{x}$ $\left.\mathrm{CH}_{2}\right), 34.17(\mathrm{CH}), 32.62,31.69,27.25,25.52\left(4 \times \mathrm{CH}_{2}\right), 25.07$ $\left(\mathrm{CH}_{3}\right), 23.40\left(\mathrm{CH}_{2}\right), 10.82(\mathrm{CH}), 3.17\left(\mathrm{CH}_{2}\right)$. HPLC: 99\%, tR 1.41 min. MS (ESI+): m/z 740.1 [M+H] $]^{+}, 370.8[\mathrm{M}+2 \mathrm{H}]^{2+}$.

5-(Benzyloxy)isoindoline (39); Prepared by $O$-benzylation of tert-butyl 5-hydroxyisoindoline-2-carboxylate $(0.590 \mathrm{~g}, 2.51$ mmol, 1 eq) using condition (e). The resultant oil was dissolved in dry DCM $(20 \mathrm{~mL})$ and trifluoroacetic acid (TFA, $2 \mathrm{~mL}$ ) was added. The solution was stirred at room temperature for approximately $2 \mathrm{~h}$. The solvent was evaporated and the residue was purified by automated flash chromatography using a gradient elution of 0 $10 \%$ of $\left[7 \mathrm{~N} \mathrm{NH}_{3}\right.$ in $\left.\mathrm{MeOH}\right]$ in DCM (and using ninhydrin TLC stain to visualize the product) to give compound $39(0.500 \mathrm{~g}, 2.22$ mmol, $89 \%)$ as a brown solid. ${ }^{1} \mathrm{H}$ NMR $\left(400 \mathrm{MHz}, \mathrm{CDCl}_{3}\right) \delta$ 7.47-7.29 (m, 5H), $7.14(\mathrm{~d}, J=8.1 \mathrm{~Hz}, 1 \mathrm{H}), 6.89-6.82(\mathrm{~m}, 2 \mathrm{H})$, $5.06(\mathrm{~s}, 2 \mathrm{H}), 4.20(\mathrm{~s}, 2 \mathrm{H}), 4.18(\mathrm{~s}, 2 \mathrm{H}) .{ }^{13} \mathrm{C}$ NMR $(101 \mathrm{MHz}$, $\left.\mathrm{CDCl}_{3}\right) \delta 158.34,143.15,137.15,133.77(4 \times \mathrm{C}), 128.61,128.58$, $127.95,127.45,122.92,113.82,108.87(6 \times \mathrm{CH}), 70.31,53.05$, $52.29\left(3 \times \mathrm{CH}_{2}\right)$. HPLC: tR $1.28 \mathrm{~min}$. MS $(\mathrm{ESI}+): \mathrm{m} / \mathrm{z} 226.4$ $[\mathrm{M}+\mathrm{H}]^{+}$.

\section{6-(3-(5-(benzyloxy)isoindolin-2-yl)propoxy)-5-} (cyclopropylmethoxy)-3,3-dimethyl-3H-indol-2-amine $\quad(40)$; Prepared using general conditions (f) and (i) from alkyl chloride 19 and isoindoline 39. (63\%) while solid. ${ }^{1} \mathrm{H}$ NMR (400 MHz, $\left.\mathrm{CDCl}_{3}\right) \delta 7.44-7.28(\mathrm{~m}, 5 \mathrm{H}), 7.10(\mathrm{~d}, J=8.0 \mathrm{~Hz}, 1 \mathrm{H}), 6.86-6.79$ (m, 4H), $5.04(\mathrm{~s}, 2 \mathrm{H}), 4.12(\mathrm{t}, J=6.2 \mathrm{~Hz}, 2 \mathrm{H}), 3.97(\mathrm{~s}, 2 \mathrm{H}), 3.95$ $(\mathrm{s}, 2 \mathrm{H}), 3.81(\mathrm{~d}, J=6.9 \mathrm{~Hz}, 2 \mathrm{H}), 2.97(\mathrm{t}, J=7.4 \mathrm{~Hz}, 2 \mathrm{H}), 2.17-$ $2.08(\mathrm{~m}, 2 \mathrm{H}), 1.45(\mathrm{~s}, 6 \mathrm{H}), 1.32-1.22(\mathrm{~m}, 1 \mathrm{H}), 0.63-0.57(\mathrm{~m}, 2 \mathrm{H})$, 0.36-0.31 (m, 2H). ${ }^{13} \mathrm{C}$ NMR $\left(101 \mathrm{MHz}, \mathrm{CDCl}_{3}\right) \delta 178.80$, $158.28,149.68,148.70,143.87,141.78,136.70,134.09,131.96(9$ x C), 128.58, 127.92, 127.44, 123.00, 113.76, 110.99, 109.07, $104.32(8 \times \mathrm{CH}), 75.96,70.27,67.63,59.34,58.59,52.90,48.37$ (C), $28.58\left(\mathrm{CH}_{2}\right), 25.05\left(\mathrm{CH}_{3}\right), 10.66(\mathrm{CH}), 3.21\left(\mathrm{CH}_{2}\right)$. HPLC: $\mathrm{t}_{\mathrm{R}}$ $1.50 \mathrm{~min}$. MS (ESI+): $\mathrm{m} / \mathrm{z} 512.4[\mathrm{M}+\mathrm{H}]^{+}$.

Conditions (k); $\begin{gathered}\text { Synthesis of } \\ \text { aminobutoxy)isoindolin-2-yl)propoxy)-5- }\end{gathered}$
(cyclopropylmethoxy)-3,3-dimethyl-3H-indol-2-amine
Palladium ( $10 \%$ on carbon ( $50 \%$ wet), 0.2 weight eq) was added to a solution of benzyl aryl ether $40(0.128 \mathrm{~g}, 0.25 \mathrm{mmol}, 1 \mathrm{eq})$ in $\mathrm{MeOH}(50 \mathrm{~mL})$. The system was flushed with $\mathrm{N}_{2}$, followed by $\mathrm{H}_{2}$ and the mixture was stirred at room temperature under $\mathrm{H}_{2}$ for 18 h. The catalyst was removed by filtration through a bed of celite ${ }^{\circledR}$ and the solvent was evaporated to give the debenzylated product, which was used without further purification. Potassium carbonate (69 $\mathrm{mg}, 0.50 \mathrm{mmol}, 2 \mathrm{eq}$ ) and 4-(boc-amino)butyl bromide (71 $\mathrm{mg}, 0.28 \mathrm{mmol}, 1.1 \mathrm{eq})$ was added to the debenzylated product and the mixture was stirred in DMF $(5 \mathrm{~mL})$, at room temperature, for 48 hours. The reaction mixture was evaporated and the residue was directly loaded onto a Biotage ${ }^{\circledR}$ SNAP KP-NH column. The product was eluted with an eluent of $0-10 \% \mathrm{MeOH}$ in DCM. The resultant white solid was dissolved in dry DCM $(20 \mathrm{~mL})$ and trifluoroacetic acid (TFA, $2 \mathrm{~mL}$ ) was added. The solution was stirred at room temperature for approximately $2 \mathrm{~h}$. The solvent was evaporated and the residue was purified by automated flash chromatography using a gradient elution of $0-10 \%$ of [ $7 \mathrm{~N} \mathrm{NH}_{3}$ in $\mathrm{MeOH}]$ in DCM (and using ninhydrin TLC stain to visualize the product) to give compound $41(12 \mathrm{mg}, 0.02 \mathrm{mmol}, 12 \%)$ as a white solid. ${ }^{1} \mathrm{H}$ NMR $\left(400 \mathrm{MHz}, \mathrm{CDCl}_{3}\right) \delta 7.07(\mathrm{~d}, J=8.2 \mathrm{~Hz}$, $1 \mathrm{H}), 6.83(\mathrm{~s}, 1 \mathrm{H}), 6.80-6.69(\mathrm{~m}, 3 \mathrm{H}), 4.12(\mathrm{t}, J=6.3 \mathrm{~Hz}, 2 \mathrm{H})$, $3.98-3.84(\mathrm{~m}, 6 \mathrm{H}), 3.81(\mathrm{~d}, J=6.9 \mathrm{~Hz}, 2 \mathrm{H}), 2.92(\mathrm{t}, J=7.3 \mathrm{~Hz}$, $3 \mathrm{H}), 2.77(\mathrm{t}, J=7.1 \mathrm{~Hz}, 2 \mathrm{H}) .{ }^{13} \mathrm{C}$ NMR $\left(101 \mathrm{MHz}, \mathrm{CDCl}_{3}\right) \delta$ $179.20,158.34,150.09,147.83,144.34,141.59,133.05,132.13(8$ x C), 122.88, 113.22, 111.41, 108.65, 103.56 (5 x CH), 76.33, $68.00,67.55,59.37,58.61,52.96\left(6 \times \mathrm{CH}_{2}\right), 48.70(\mathrm{C}), 41.88$, $30.20,28.92,26.70\left(4 \times \mathrm{CH}_{2}\right), 25.08\left(\mathrm{CH}_{3}\right), 10.81(\mathrm{CH}), 3.18$ (CH2). HPLC: $t_{R} 1.15 \mathrm{~min} . \mathrm{MS}(\mathrm{ESI}+): \mathrm{m} / \mathrm{z} 493.6[\mathrm{M}+\mathrm{H}]^{+}$.

Construct design, cloning, expression and purification of human SPINDLIN proteins

A plasmid encoding full length human SPIN1 was obtained from Source Bioscience (IOH9972-pDEST26) and used as a template to clone SPIN1M26-S262 and SPIN1P49-S262 into the pNIC$\mathrm{CTHF}$ vector with a TEV (tobacco etch virus) cleavable Cterminal His6 tag. SPIN1G21-S262 was cloned into the pNIC-Bio2 vector with a TEV cleavable N-terminal His 10 tag, and a Cterminal biotinylation sequence. SPIN2BP45-S258, SPIN3M27-S258 and SPIN4 ${ }_{\text {T36-P249 }}$ were cloned into the pNIC vector with a TEV cleavable N-terminal His6 tag using templates obtained from the Mammalian Gene Collection and Source Bioscience (SPIN2B: cDNA clone IMAGE id:6729986, SPIN3 IMAGENE: IRCBp5005F0211Q and SPIN4 IMAGE id 40032302). For crystallization studies SPIN2BP45-S258 and SPIN3P45-S258 were also cloned into the pNIC-CTHF vector. The recombinant proteins were expressed in a phage-resistant derivative of Escherichia coli strain BL21(DE3) carrying the pRARE2 plasmid for rare codon expression. Cells were grown at $37{ }^{\circ} \mathrm{C}$ in Terrific Broth supplemented with $50 \mu \mathrm{g} / \mathrm{ml}$ kanamycin and $34 \mu \mathrm{g} / \mathrm{ml}$ chloramphenicol, until the culture reached an $\mathrm{O}_{600}$ of 2.0. The temperature was decreased to $18{ }^{\circ} \mathrm{C}$ and protein expression induced with $0.1 \mathrm{mM}$ IPTG (isopropyl $\beta$-D-thiogalactopyranoside) overnight. Cells were collected by centrifugation and frozen at $-80^{\circ} \mathrm{C}$. Biotinylated SPIN1G21-S262 was expressed similarly in a BL21(DE3)-R3pRARE2-BirA strain as described in reference $4 .{ }^{64}$ For purification, cells were resuspended in $50 \mathrm{mM}$ Hepes $\mathrm{pH} 7.5,500 \mathrm{mM}$ $\mathrm{NaCl}, 10 \mathrm{mM}$ imidazole, $5 \%$ glycerol, $0.5 \mathrm{mM}$ TCEP and a protease inhibitor cocktail (Sigma), and lysed by sonication. The cell lysate was clarified by centrifugation and the proteins purified by nickel-affinity chromatography (GE Healthcare) using a stepwise gradient of imidazole. The histidine tag was removed by incubating SPIN1P49-S262, SPIN1G21-S262, SPIN2BP45-S258, SPIN3P45-S258 and SPIN4T36-P249 with TEV protease at $4{ }^{\circ} \mathrm{C}$ overnight and this was followed by size exclusion chromatography (Superdex 75 or Superdex 200, GE Healthcare) in $10 \mathrm{mM}$ Hepes pH 7.5, $500 \mathrm{mM}$ $\mathrm{NaCl}, 5 \%$ glycerol and $0.5 \mathrm{mM}$ TCEP. The TEV protease and the uncleaved proteins were removed by nickel-affinity chromatography and concentrated using an Amicon centrifugal filtration unit. Mass of purified proteins was verified by electrospray ionization time of flight mass spectrometry (ESI-TOF-TOF: Agilent LC/MSD).

\section{Crystallization and $\mathrm{X}$-ray data collection}

Proteins used for crystallization studies had the histidine tag removed, and all proteins were crystallized at $4{ }^{\circ} \mathrm{C}$ using the sitting drop vapor diffusion method. SPIN1 Pro49-Ser262 was incubated with $4.5 \mathrm{mM}$ A-366 at $31 \mathrm{mg} / \mathrm{ml}$ and crystals were obtained in a drop containing $100 \mathrm{nl}$ of protein compound mixture, $30 \mathrm{nl}$ precipitant consisting of $0.1 \mathrm{M}$ Bis-Tris pH $6.5,0.2 \mathrm{M} \mathrm{CaCl}_{2}, 45 \%$ MPD and $20 \mathrm{nl}$ seeds obtained from crystals grown in $0.1 \mathrm{M}$ BisTris $\mathrm{pH} 5.5,0.1 \mathrm{M} \mathrm{MgCl}_{2}$ and $16 \%$ PEG3350. The crystal was cryo protected in precipitant solution supplemented with $25 \%$ 
ethylene glycol before it was flash cooled in liquid nitrogen. SPIN1 Pro49-Ser262 was incubated with $1.5 \mathrm{mM}$ of either compound $\mathbf{2 3}$ or compound $\mathbf{3 5}$ using $39 \mathrm{mg} / \mathrm{ml}$ protein. Crystals appeared in drops consisting of $75 \mathrm{nl}$ protein compound mixture and $75 \mathrm{nl}$ precipitant consisting of 55-60\% MPD and 0.1 M SPG (Succinate, Phosphate and Glycine) buffer $\mathrm{pH}$ 5.5-6.0. SPIN2BP45-S258 was crystallized using $23 \mathrm{mg} / \mathrm{ml}$ protein preincubated with 3-fold molar excess of a ARTK(me3)QTAR(me2a)KS peptide. Crystals appeared in drops consisting of $100 \mathrm{nl} \mathrm{SPIN2B/peptide} \mathrm{and} 50 \mathrm{nl}$ precipitant composed of $0.1 \mathrm{M}$ Bis-Tris buffer $\mathrm{pH} 5.5$ and $25 \%$ PEG3350. SPIN3P45-S258 was crystallized in a drop consisting of $75 \mathrm{nl} 24 \mathrm{mg} / \mathrm{mL}$ protein, $55 \mathrm{~nL}$ precipitant compost of of $0.1 \mathrm{M}$ Tris buffer $\mathrm{pH} 8.4$ and 38\% PEG3350, and 20nl seeds prepared from crystals grown in the same condition. SPIN4T36-P249 was crystallized using $29 \mathrm{mg} / \mathrm{mL}$ protein preincubated with $1.7 \mathrm{mM}$ ARTK(Me3)QTARKS peptide. Crystals were obtained in drops consisting of $50 \mathrm{nl}$ protein /peptide, $80 \mathrm{nl}$ precipitant compost of $30 \%$ PEG3350 and 0.1 M HEPES pH 7.0 and $20 \mathrm{nl}$ seeds prepared from crystals grown in the same condition. Crystals of SPIN2B, SPIN3 and SPIN4 were cryo protected in precipitant solution supplemented with $25 \%$ ethylene glycol before they were flash cooled in liquid nitrogen. All data were collected on beamlines I02, I03 and I24 at the Diamond Light source UK.

\section{Structure determination}

All datasets were processed, scaled and merged at the Diamond light source using Xia2, autoPROC, ${ }^{65} \mathrm{XDS},{ }^{66}$ POINTLESS,${ }^{67}$ AIMLESS, ${ }^{68}$ and CCP4. ${ }^{69}$ Electron density maps were obtained by molecular replacement using PHASER with previously determined structures of SPIN1 as a search model (pdb id 4H75 and $4 \mathrm{MZG}$ ). The complex structure of SPIN1 with A-366 was solved to $1.52 \AA$ resolution (pdb 6I8Y), with compound 23 to $1.58 \AA$ (pdb 6I8L) and with compound 35 to $1.76 \AA$ resolution (pdb 6I8B). SPIN2B was solved to $1.7 \AA$ (pdb 5LUG), SPIN3 to $2.0 \AA$ (pdb $5 \mathrm{~A} 1 \mathrm{H})$ and SPIN4 to $1.86 \AA$ resolution. All structures were refined in an iterative process combining REFMAC and PHENIX ${ }^{70}$ with electron density map inspections and model improvement in $\mathrm{COOT}^{71}$ and terminated when there were no significant changes in the $R_{\text {work }}$ and $R_{\text {free }}$ values, and inspection of the electron density map suggested that no further corrections or additions were justified. Structural analysis and figures were performed with PyMOL (http://www.pymol.org).

\section{AlphaScreen Assay}

Compound binding was determined by the displacement of a trimethylated biotinylated histone $\mathrm{H} 3$ peptide from a Hexa His tagged SPIN1 Tudor domain protein using an AlphaScreen ${ }^{\circledR}$ Histidine (Nickel Chelate) Detection Kit. Compound was dispensed from DMSO stocks into assay plates (ProxiPlate-384 Plus, Perkin Elmer) using an Echo 525 Liquid Handler (Labcyte). Assays were performed at $25{ }^{\circ} \mathrm{C}$ in a volume of $20 \mu \mathrm{L}$ in a buffer that was 25 mM HEPES pH7.5, $100 \mathrm{mM} \mathrm{NaCl}, 0.1 \%$ BSA, $0.05 \%$ CHAPS with $12.5 \mathrm{nM}$ peptide (ARTK(me3)QTARKSTGGKAPRKbiotin), $12.5 \mathrm{nM}$ SPIN1, $0.2 \%$ DMSO. Peptide and protein (SPIN1 ${ }_{\mathrm{M} 26-\mathrm{S} 262}$ C-terminal His6 tag) were pre-mixed, added to the compound plate and incubated for 30 minutes after which AlphaScreen ${ }^{\circledR}$ beads were added to a final concentration of 2.5 $\mu \mathrm{g} / \mathrm{mL}$. Assay plates were incubated for 60 minutes then luminescence was measured using a Pherastar FS/FSX (BMG).

\section{Bio-Layer Interferometry (BLI) Assay}

The direct interaction between small molecules and SPIN1 was measured using a BLI Octet RED384 (FortéBio Inc., Menlo Park, CA). Superstreptavidin biosensors (FortéBio Inc.) were coated in a solution containing $50 \mu \mathrm{g} / \mathrm{mL}$ of biotinylated protein $\left(\mathrm{SPIN} 1_{\mathrm{G} 21-}\right.$ S262 C-terminal biotinylation) for $5 \mathrm{~min}$ at $25^{\circ} \mathrm{C}$. Compound was dispensed from DMSO stocks into plates using an Echo 525 Liquid Handler (Labcyte). Assays were performed at $25^{\circ} \mathrm{C}$ in a vol- ume of $60 \mu \mathrm{L}$ in a buffer that was $25 \mathrm{mM}$ HEPES $\mathrm{pH} 7.5,150$ $\mathrm{mM} \mathrm{NaCl}$ and $0.05 \%$ TWEEN 20. A duplicate set of sensors without protein was used as a background binding control. Association of samples $(5000,2500,1250,625,312.5,156.25$ and $78.125 \mathrm{nM}$ ) to coated and uncoated reference sensors was measured over 120 seconds and dissociation over 240 seconds. Data analysis on the FortéBio Octet RED96 instrument was performed using a double reference subtraction (sample and sensor references) in the FortéBio data analysis software, which accounts for nonspecific binding, background, and signal drift and minimizes well-based and sensor variability, while $\mathrm{Kd}$ values were fitted using a 1:1 model. try

Thermal Shift Assay (TSA)/Differential Scanning Fluorime-

TSA/DSF experiments for UHRF1, 53BP1, TDRD3, SND1, SETDB1 and SGF29 were carried out as reported by Neisen et al. ${ }^{29}$ All other TSA/DSF experiments were performed on a Lightcycler480 (Roche Molecular Systems). Compounds were dispensed into 384-well PCR plates ( $20 \mu \mathrm{l}$ assay volume) to a final concentration of $20 \mu \mathrm{M}$. Purified proteins were diluted to $2 \mathrm{uM}$ in buffer (20 mM HEPES, $500 \mathrm{mM}, \mathrm{pH}$ 7.5). SYPRO orange dye was added to a 1:1000 dilution from purchased stock (LifeTechnologies). Proteins were melted over a gradient from 20 to $95{ }^{\circ} \mathrm{C}$ with three acquisitions per cycle $\left(0.19^{\circ} \mathrm{C} / \mathrm{s}\right)$. The shift in unfolding $\left(\mathrm{D} T_{\mathrm{m}}\right)$ was calculated as the difference between the mean melting temperature $\left(T_{\mathrm{m}}\right)$ for DMSO reference wells $(\mathrm{n}=16$ per protein) and $T_{\mathrm{m}}$ for the compound wells (duplicates for each compound). $T_{\mathrm{m}}$ was estimated by fitting a sigmoidal Boltzmann equation to the fluorescence intensity $I(T)$ over the unfolding process:

$$
\left.I(T)=I_{\text {onset }}+\left(I_{\text {end }}-\text { Ibaseline }\right) / 1+\mathrm{e}^{\wedge}\left(\left(T_{\mathrm{m}}-T\right) / S\right)\right)
$$

where $I_{\mathrm{onset}}$ is the intensity before the onset of unfolding, $I_{\mathrm{end}}$ is the intensity at the endpoint of unfolding and $S$ is the slope of the curve.

\section{Isothermal Titration Calorimetry}

Purified protein was diluted (see SI Table S5) in ITC buffer (100 mM NaCl, $20 \mathrm{mM}$ Tris, $\mathrm{pH} \mathrm{8.0)}$ and then dialyzed against a 1000 times excess of ITC buffer at $4{ }^{\circ} \mathrm{C}$ overnight using SnakeSkin ${ }^{\circledR}$ Dialysis Tubing (Thermo Scientific) with a $3.5 \mathrm{kDa}$ molecular weight cut off. The dialyzed sample was then centrifuged at $14.000 \mathrm{rpm}$ in a standard chilled benchtop centrifuge at $4{ }^{\circ} \mathrm{C}$ to remove aggregate. Protein concentration (see SI Table S5) was then verified via absorption at $280 \mathrm{~nm}$ using a NanoDrop ND1000 Spectrophotometer. Compounds were diluted in ITC buffer (SI, Table S5). ITC was performed on a NanoITC Standard Volume instrument (TA Instruments) using direct titration (protein in cell, compound in syringe) at $20{ }^{\circ} \mathrm{C}$ with an initial injection of $3.7 \mu \mathrm{L}$ followed by 30 injections of $7.96 \mu \mathrm{L}$ at a stir rate of $350 \mathrm{rpm}$. Data were analyzed using an independent fit model with the NanoAnalyse software (TA Instruments, version 3.4.0).

\section{Development of SPIN1 Nanobret assay}

U20S cell $(2.8 \times 105)$ were plated in each well of a 6-well plate. After $6 \mathrm{~h}$ cells were co-transfected with C-terminal HaloTagHistone 3.3 (NM_002107) and an N-terminal NanoLuciferase fusion of full length SPIN1 at a 1:500 (NanoLuc ${ }^{\circledR}$ to HaloTag $\left.{ }^{\circledR}\right)$ ratio respectively with FuGENE HD transfection regent. Sixteen hours post-transfection, cells were collected, washed with PBS, and exchanged into media containing phenol red-free DMEM and $4 \%$ FBS in the absence (control sample) or the presence (experimental sample) of $100 \mathrm{nM}$ NanoBRET 618 fluorescent ligand (Promega). Cells were then re-plated in a 384-well assay white plate (Greiner \#3570) at 2.7 $\times 103$ cells per well. Compounds were then added directly to media at final concentrations of $0-30 \mu \mathrm{M}$ or an equivalent amount of DMSO as a vehicle control, and the plates were incubated for $24 \mathrm{~h}$ at $37{ }^{\circ} \mathrm{C}$ in the presence of $5 \%$ 
$\mathrm{CO}_{2}$. NanoBRET Nano-Glo substrate (Promega) was added to both control and experimental samples at a final concentration of $10 \mu \mathrm{M}$. Readings were performed within 10 minutes using a ClarioSTAR (BMG labtech). A corrected BRET ratio was calculated and is defined as the ratio of the emission at $610 \mathrm{~nm} / 460 \mathrm{~nm}$ for experimental samples minus the emission at $610 \mathrm{~nm} / 460 \mathrm{~nm}$ for control samples (without NanoBRET fluorescent ligand). BRET ratios are expressed as milliBRET units $(\mathrm{mBU})$, where $1 \mathrm{mBU}$ corresponds to the corrected BRET ratio multiplied by 1000 . The assay was further validated by domain specific site directed mutagenesis (Y170A) ablating peptide and ligand binding.

\section{osis}

SCC040 cells were seeded into 96-well (proliferation) or 6 well plates (cell cycle and apoptosis) at a density of 500 and 15,000 cells/well respectively in DMEM medium (supplemented with $10 \%$ FCS, $2 \mathrm{mM}$ L-Glutamine, 100 units $/ \mathrm{mL}$ penicillin, 100 $\mathrm{ug} / \mathrm{mL}$ streptomycin, $1 \mathrm{x}$ NEAA) in the presence of $1 \mu \mathrm{M}, 2 \mu \mathrm{M}$ and $4 \mu \mathrm{M}$ compound $\mathbf{3 5}, \mathbf{3 8}$ or DMSO control. To assess proliferation cells were measured for confluence, using brightfield imaging on the Celigo (Nexcelome) and direct cell counting of cell nuclei within each well $1 \mathrm{~h}$ by incubation of Hoechst 33342 at 10 $\mu \mathrm{g} / \mathrm{mL}$ at day 4 and day 6 after seeding. To assess cell cycle parameters, cells were trypsinized, pelleted and re-suspended in 0.5 $\mathrm{mL} \mathrm{SSC040} \mathrm{media} \mathrm{with} 10 \mu \mathrm{g} / \mathrm{mL}$ Hoechst 33342 for $45 \mathrm{~min}$ at $37{ }^{\circ} \mathrm{C}, 5 \% \mathrm{CO}_{2}$. Cells were then analyzed on a BD LSRFortessa ${ }^{\mathrm{TM}}$ and cell cycle measured using FlowJoV10. To assess apoptosis cells the Dead Cell Apoptosis Kit with Annexin V Alexa Fluor ${ }^{\mathbb{B}}$ 488 and PI (Invitrogen) kit was used. In brief, cells were trypsinized, washed in PBS, pelleted and re-suspended in $0.1 \mathrm{~mL} 1 \mathrm{x}$ Annexin Binding buffer. $1 \mu \mathrm{L}$ of $100 \mu \mathrm{L} / \mathrm{mL}$ Propidium Iodide (PI) and $5 \mu$ Alexa Fluor ${ }^{\circledR} 488$ annexin V was added per sample and incubated at room temperature for $15 \mathrm{~min}$ in the dark. $0.4 \mathrm{~mL}$ Annexin Binding buffer was then added to each sample and cells were then analyzed on a BD LSRFortessa ${ }^{\mathrm{TM}}$ and apoptosis measured using FlowJoV10.

\section{Chemoproteomic - Preparation of affinity resin}

Amine functionalized compound $\mathbf{4 1}$ was coupled to NHSactivated Sepharose 4 fast flow beads (GE Healthcare). ${ }^{72} 100 \mu \mathrm{L}$ of bead slurry ( $50 \%$ in isopropanol) were used for each pull-down experiment. Beads were washed with DMSO $(500 \mu \mathrm{L})$, collected by centrifugation ( $3 \mathrm{~min}, 60 \mathrm{x} \mathrm{g}$ ), and the supernatant removed. After three wash cycles, the beads were resuspended in DMSO $(50 \mu \mathrm{L})$, to which the amine $(0.025 \mu \mathrm{mol})$ and triethylamine $(0.75$ $\mu \mathrm{L})$ were added. The beads were incubated at room temperature for 16 hours, and depletion of free amine from the supernatant determined by LC-MS analysis. Ethanolamine $(2.5 \mu \mathrm{L})$ was then added to block any unreacted NHS sites, and the beads incubated for a further 16 hours. Derivatized beads were then washed with DMSO $(3 \times 500 \mu \mathrm{L})$, Buffer A $(50 \mathrm{mM}$ Tris $\mathrm{pH} 7.5,0.8 \% \mathrm{v} / \mathrm{v}$ $\mathrm{NP}-40,5 \%$ v/v glycerol, $1.5 \mathrm{mM} \mathrm{MgCl}, 100 \mathrm{mM} \mathrm{NaCl}, 25 \mathrm{mM}$ $\mathrm{NaF}, 1 \mathrm{mM} \mathrm{Na} \mathrm{VO}_{4}, 1 \mathrm{mM}$ PMSF, $1 \mathrm{mM}$ DTT, $10 \mu \mathrm{g} / \mathrm{mL}$ TLCK, $1 \mu \mathrm{g} / \mathrm{mL}$ Leupeptin, $1 \mu \mathrm{g} / \mathrm{mL}$ Aprotinin, $1 \mu \mathrm{g} / \mathrm{mL}$ soy bean tryp$\sin )(3 \times 1 \mathrm{~mL})$. Resin was incubated with cell lysates $(2 \mathrm{mg}$ of protein per pulldown, at $6 \mathrm{mg} / \mathrm{mL}$ ) that had been pre-treated with either compound 35, $38(20 \mu \mathrm{M})$ or DMSO control for 30 minutes at $4{ }^{\circ} \mathrm{C}$. Beads and treated lysates were incubated for 2 hours at 4 ${ }^{\circ} \mathrm{C}$, before being washed with Buffer A $(5 \mathrm{~mL})$ proteins were eluted by addition of $80 \mu \mathrm{L}$ of $2 \mathrm{x}$ sample Buffer $(65.8 \mathrm{mM}$ Tris$\mathrm{HCl} \mathrm{pH} \mathrm{6.8,} \mathrm{26.3 \%} \mathrm{(w/v)} \mathrm{glycerol,} \mathrm{2.1 \%} \mathrm{SDS,} \mathrm{0.01 \%} \mathrm{bromophe-}$ nol blue, $50 \mathrm{mM}$ DTT). Before MS analysis, SPIN1 pull-down was verified by Western Blotting using primary antibody ab118784 (Abcam UK, rabbit) at 1:1000.

\section{MS sample preparation}

Samples were reduced with DTT (10 mM final concentration) for 30 minutes at room temperature, alkylated with iodoacetamide (55 mM final concentration) for 30 minutes at room temperature, diluted with $\mathrm{TEAB}$, and incubated with trypsin $(6 \mu \mathrm{L}, 0.2 \mathrm{mg} / \mathrm{mL})$ overnight at $37{ }^{\circ} \mathrm{C}$. The digests were then desalted using SEPAC lite columns (Waters), eluted with $69 \% \mathrm{v} / \mathrm{v} \mathrm{MeCN}, 0.1 \% \mathrm{v} / \mathrm{v} \mathrm{FA}$ in $\mathrm{H}_{2} \mathrm{O}(1 \mathrm{~mL})$ and dried in vacuo. Dried peptides were stored at $20{ }^{\circ} \mathrm{C}$ before resuspension in $2 \% \mathrm{~V} / \mathrm{V} \mathrm{MeCN}, 0.1 \% \mathrm{v} / \mathrm{v} \mathrm{FA}$ in $\mathrm{H}_{2} \mathrm{O}(20 \mu \mathrm{L})$ for LC-MS/MS analysis. Mass spectrometry data was acquired at the Discovery Proteomics Facility (University of Oxford). Digested samples were analyzed by nano-UPLCMS/MS using a Dionex Ultimate 3000 nano UPLC with EASY spray column $(75 \mu \mathrm{m} \times 500 \mathrm{~mm}, 2 \mu \mathrm{m}$ particle size, Thermo Scientific) with a $60 \mathrm{~min}$ gradient of $0.1 \%(\mathrm{v} / \mathrm{v})$ formic acid in $5 \%$ (v/v) DMSO to $0.1 \%(\mathrm{v} / \mathrm{v})$ formic acid with $35 \%(\mathrm{v} / \mathrm{v})$ acetonitrile in $5 \%(\mathrm{v} / \mathrm{v})$ DMSO at a flow rate of approximately $250 \mathrm{~nL} / \mathrm{min}$ (600 bar per $40{ }^{\circ} \mathrm{C}$ column temperature). Mass spectrometry data was acquired with an Orbitrap Q Exactive High Field (HF) instrument (ThermoFisher). Survey scans were acquired at a resolution of 60,000 at $400 \mathrm{~m} / \mathrm{z}$ and the 20 most abundant precursors were selected for CID fragmentation.

\section{Data analysis}

Raw data was processed using MaxQuant version 1.5.0.253 and the reference complete human proteome FASTA file (UniProt). Label Free Quantification (LFQ) and Match Between Runs were selected; replicates were collated into parameter groups to ensure matching between replicates only. Cysteine carbamidomethylation was selected as a fixed modification, and methionine oxidation as a variable modification. Default settings for identification and quantification were used. Specifically, a minimum peptide length of 7, a maximum of 2 missed cleavage sites and a maximum of 3 labelled amino acids per peptide were employed. Peptides and proteins were identified utilizing a 0.01 false discovery rate, with "Unique and razor peptides" mode selected for both identification and quantification of proteins (razor peptides are uniquely assigned to protein groups and not to individual proteins). At least 2 razor + unique peptides were required for valid quantification. Processed data was further analyzed using Perseus version 1.5.0.9 and Microsoft Excel 2010. Peptides categorized by MaxQuant as 'potential contaminants', 'only identified by site' or 'reverse' were filtered, and the LFQ intensities transformed by $\log$ 2. Experimental replicates were grouped, and two valid LFQ values were required in at least one experimental group. Missing values were imputed using default settings, and the data distribution visually inspected to ensure that a normal distribution was maintained. Statistically significant competition was determined through the application of P2 tests, using a permutation-based FDR of 0.05 and an S0 of 2, and data were visualized in volcano plots.

The accompanying SI Data Excel file contains a summary of the chemoproteomic data.

Transcriptional responses to SPIN1 inhibitor and siRNA knockdown in squamous cell carcinoma cell lines

SSC040 cells were seeded into wells of a 48 -well plate at a density of 10,000 cells/well in DMEM medium (supplemented with $10 \%$ FCS, $2 \mathrm{mM}$ L-Glutamine, 100 units $/ \mathrm{mL}$ penicillin, 100 $\mathrm{ug} / \mathrm{mL}$ streptomycin, 1x NEAA). Compounds (35 and $\mathbf{3 8}$ or DMSO control) or siRNA were added 24 hours later. RNA was harvested using Trizol reagent, 2 and 4 days after addition of siRNA, or 5 and 7 days after addition of compounds. RNA was purified using a Direct-zol RNA miniprep kit (Zymo Research). Experiments were conducted in 2 biological replicates, with $n=3$ technical replicates per condition.

RNAseq, library preparation, sequencing 
RNA concentration derived from inhibitor experiments $(n=3$, technical replicates) was normalized to $100 \mathrm{ng} / 50 \mathrm{uL}$ and Illumina libraries were prepared using a NEBNext Poly(A) mRNA Magnetic Isolation Module in conjunction with a NEBNext Ultra II Directional RNA Library Prep Kit (New England Biolabs), according to the manufacturer's instructions. Libraries were quantitated using the Agilent High Sensitivity D1000 ScreenTape System on a 2200 Tapestation (Agilent), pooled at equimolar concentration, denatured and sequenced on a NextSeq 500 platform (Illumina).

\section{NGS data processing and analysis}

Sequencing reads from the RNAseq experiment were aligned to the human genome (hg38) using HISAT ${ }^{73}$ with default parameters. The program featureCounts ${ }^{74}$ was used to assign mapped reads to genes with annotation (gtf file Ensembl87). Differential gene expression analysis was performed using DESeq $2^{75}$ with an FDR-adjusted $p$ value $<0.05$ and $\log 2$ fold change $>1$. Functional analysis of differentially expressed genes was performed using Reactome. $^{76}$

\section{Statistical methods and data analysis}

All statistical analyses and data fitting were performed using GraphPad Prism software version 7. Data were analyzed using two-sided $t$ tests or one-way ANOVA, unless otherwise stated, and as indicated in the figure legends with a p-value $<0.05$. Statistical analysis of the sequencing data, data collection and structure refinement was automatically performed in all of the programs used as noted above. The number of sampled units (n) as well as the number of technical replicates is indicated in the figure legends.

The accompanying SI Data Excel file contains the RNAseq data

\section{ASSOCIATED CONTENT}

\section{Supporting Information}

The Supporting Information is available free of charge on the ACS Publications website.

X-ray crystallographic data; analysis of ligand binding and sequence alignment of SPIN family proteins; biophysical and biochemical assays results; cellular assay results; browser track for selected genomic regions; results of in vitro stability, permeability and solubility assays; NMR spectra (with assignments) and HPLC chromatograms of end compounds. (PDF)

Chemical structures, molecular formula strings; chemoproteomic data summary; RNAseq results and reactome analysis. (XLS)

\section{AUTHOR INFORMATION}

\section{Corresponding Author}

*E-mail: paul.brennan@sgc.ox.ac.uk.

* E-mail: udo.oppermann@sgc.ox.ac.uk

\section{Author Contributions}

The manuscript was written through contributions of all authors. All authors have given approval to the final version of the manuscript. $\$$ These authors contributed equally.

\section{ACKNOWLEDGMENT}

The work was supported by Cancer Research UK (C41580/A23900), Oxford NIHR Biomedical Research Centre, Myeloma UK, Arthritis Research UK (20522) and Alzheimer's Research UK (ARUK-2018DDI-OX). The SGC (charity no. 1097737) receives funds from AbbVie, Bayer Pharma AG,
Boehringer Ingelheim, Canada Foundation for Innovation, Eshelman Institute for Innovation, Genome Canada, Innovative Medicines Initiative (EU/EFPIA) [ULTRA-DD grant no. 115766], Janssen, Merck KGaA Darmstadt Germany, MSD, Novartis Pharma AG, Ontario Ministry of Economic Development and Innovation, Pfizer, São Paulo Research Foundation-FAPESP, Takeda, Wellcome [106169/ZZ14/Z]. Funding was received from People Programme (Marie Curie Actions) of the European Union's Seventh Framework Programme (FP7/2007-2013) under REA grant agreement $n^{\circ}$ [609305]. JJ received support from grants R01CA218600, R01CA230854, R01GM122749 and R01HD088626 from the US National Institutes of Health. The views expressed are the author(s) and not necessarily those of the NHS, NIHR or Department of Health.

\section{ABBREVIATIONS}

SPIN1 (Spindlin1), DSF (differential scanning fluorimetry), SPA (scintillation proximity assay), ITC (isothermal titration calorimetry), CPP (chemical probe pairing), HNSCC (head and neck squamous cell carcinoma), SCC (squamous cell carcinoma), CRI (cancer-related inflammation).

\section{PROTEIN DATA BANK}

A-366 in complex with SPIN1 PDB ID 6I8Y; Compound 23 in complex with SPIN1 PDB ID 6I8L; Compound 35 in complex with SPIN1 PDB ID 6I8B

\section{REFERENCES}

(1) Jenuwein, T.; Allis, C. D., Translating the histone code. Science 2001, 293 (5532), 1074-1080.

(2) Su, Z.; Denu, J. M., Reading the combinatorial histone language. ACS Chem. Biol. 2016, 11 (3), 564-574.

(3) Teske, K. A.; Hadden, M. K., Methyllysine binding domains: structural insight and small molecule probe development. Eur. J. Med. Chem. 2017, 136, 14-35.

(4) Oh, B.; Hwang, S. Y.; Solter, D.; Knowles, B. B., Spindlin, a major maternal transcript expressed in the mouse during the transition from oocyte to embryo. Development 1997, 124 (2), 493-503.

(5) Bae, N.; Viviano, M.; Su, X.; Lv, J.; Cheng, D.; Sagum, C.; Castellano, S.; Bai, X.; Johnson, C.; Khalil, M. I.; Shen, J.; Chen, K.; Li, H.; Sbardella, G.; Bedford, M. T., Developing spindlin1 small-molecule inhibitors by using protein microarrays. Nat. Chem. Biol. 2017, 13 (7), 750-756.

(6) Fletcher, B. S.; Dragstedt, C.; Notterpek, L.; Nolan, G. P., Functional cloning of SPIN-2, a nuclear anti-apoptotic protein with roles in cell cycle progression. Leukemia 2002, 16 (8), 1507-1518.

(7) Franz, H.; Greschik, H.; Willmann, D.; Ozretic, L.; Jilg, C. A.; Wardelmann, E.; Jung, M.; Buettner, R.; Schule, R., The histone code reader SPIN1 controls RET signaling in liposarcoma. Oncotarget 2015, 6 (7), 4773-4789.

(8) Janecki, D. M.; Sajek, M.; Smialek, M. J.; Kotecki, M.; GinterMatuszewska, B.; Kuczynska, B.; Spik, A.; Kolanowski, T.; Kitazawa, R.; Kurpisz, M.; Jaruzelska, J., SPIN1 is a proto-oncogene and SPIN3 is a tumor suppressor in human seminoma. Oncotarget 2018, 9 (65), 3246632477.

(9) Staub, E.; Mennerich, D.; Rosenthal, A., The Spin/Ssty repeat: a new motif identified in proteins involved in vertebrate development from gamete to embryo. Genome Biol. 2002, 3 (1), RESEARCH0003.

(10) Jiang, F.; Zhao, Q.; Qin, L.; Pang, H.; Pei, X.; Rao, Z., Expression, purification, crystallization and preliminary X-ray analysis of human spindlin1, an ovarian cancer-related protein. Protein Pept. Lett. 2006, 13 (2), 203-205.

(11) Chen, X.; Wang, Y. W.; Xing, A. Y.; Xiang, S.; Shi, D. B.; Liu, L.; Li, Y. X.; Gao, P., Suppression of SPIN1-mediated PI3K-Akt pathway by miR-489 increases chemosensitivity in breast cancer. J. Pathol. 2016, 239 (4), 459-472.

(12) Chen, X. J.; Dong, H.; Liu, S.; Yu, L.; Yan, D. X.; Yao, X. W.; Sun, W. J.; Han, D. Z.; Gao, G. Z., Long noncoding RNA MHENCR promotes melanoma progression via regulating miR-425/489-mediated PI3K-Akt pathway. Am. J. Transl. Res. 2017, 9 (1), 90-102. 
(13) Drago-Ferrante, R.; Pentimalli, F.; Carlisi, D.; De Blasio, A.; Saliba, C.; Baldacchino, S.; Degaetano, J.; Debono, J.; Caruana-Dingli, G.; Grech, G.; Scerri, C.; Tesoriere, G.; Giordano, A.; Vento, R.; Di Fiore, R., Suppressive role exerted by microRNA-29b-1-5p in triple negative breast cancer through SPIN1 regulation. Oncotarget 2017, 8 (17), 2893928958

(14) Fang, Z. L.; Cao, B.; Liao, J. M.; Deng, J.; Plummer, K. D.; Liao, P.; Liu, T.; Zhang, W. S.; Zhang, K.; Li, L.; Margolin, D.; Zeng, S. X.; Xiong, J. P.; Lu, H., SPIN1 promotes tumorigenesis by blocking the uL18 (universal large ribosomal subunit protein 18)-MDM2-p53 pathway in human cancer. Elife 2018, 7, e31275.

(15) Li, Y.; Ma, X. L.; Wang, Y. P.; Li, G. H., miR-489 inhibits proliferation, cell cycle progression and induces apoptosis of glioma cells via targeting SPIN1-mediated PI3K/AKT pathway. Biomed. \& Pharmacother. 2017, 93, 435-443.

(16) Song, Q.; Ji, Q.; Xiao, J.; Li, F.; Wang, L.; Chen, Y.; Xu, Y.; Jiao, S., miR-409 inhibits human non-small-cell lung cancer progression by directly targeting SPIN1. Mol. Ther. Nucleic Acids 2018, 13, 154-163.

(17) Su, X. N.; Zhu, G. X.; Ding, X. Z.; Lee, S. Y.; Dou, Y. L.; Zhu, B.; Wu, W.; Li, H. T., Molecular basis underlying histone H3 lysinearginine methylation pattern readout by Spin/Ssty repeats of Spindlin1. Genes Dev. 2014, 28 (6), 622-636.

(18) Wang, J. X.; Zeng, Q.; Chen, L.; Du, J. C.; Yan, X. L.; Yuan, H. F.; Zhai, C.; Zhou, J. N.; Jia, Y. L.; Yue, W.; Pei, X. T., Spindlin1 promotes cancer cell proliferation through activation of WNT/TCF-4 signaling. Mol. Cancer Res. 2012, 10 (3), 326-335.

(19) Bartke, T.; Vermeulen, M.; Xhemalce, B.; Robson, S. C.; Mann, M.; Kouzarides, T., Nucleosome-interacting proteins regulated by DNA and histone methylation. Cell 2010, 143 (3), 470-484.

(20) Wang, W. X.; Chen, Z.; Mao, Z.; Zhang, H. H.; Ding, X. J.; Chen, S.; Zhang, X. D.; Xu, R. M.; Zhu, B., Nucleolar protein spindlin1 recognizes $\mathrm{H} 3 \mathrm{~K} 4$ methylation and stimulates the expression of rRNA genes. Embo Rep 2011, 12 (11), 1160-1166.

(21) Shanle, E. K.; Shinsky, S. A.; Bridgers, J. B.; Bae, N.; Sagum, C.; Krajewski, K.; Rothbart, S. B.; Bedford, M. T.; Strahl, B. D., Histone peptide microarray screen of chromo and tudor domains defines new histone lysine methylation interactions. Epigenet Chromatin 2017, 10, 12.

(22) Bae, N.; Gao, M.; Li, X.; Premkumar, T.; Sbardella, G.; Chen, J. J.; Bedford, M. T., A transcriptional coregulator, SPIN.DOC, attenuates the coactivator activity of spindlin1. J. Biol. Chem. 2017, 292 (51), 2080820817.

(23) Robaa, D.; Wagner, T.; Luise, C.; Carlino, L.; McMillan, J.; Flaig, R.; Schule, R.; Jung, M.; Sippl, W., Identification and structureactivity relationship studies of small-molecule inhibitors of the methyllysine reader protein spindlin1. Chemmedchem 2016, 11 (20) 2327-2338.

(24) Sweis, R. F.; Pliushchev, M.; Brown, P. J.; Guo, J.; Li, F. L.; Maag, D.; Petros, A. M.; Soni, N. B.; Tse, C.; Vedadi, M.; Michaelides, M. R.; Chiang, G. G.; Pappano, W. N., Discovery and development of potent and selective inhibitors of histone methyltransferase G9a. Acs Med. Chem. Lett. 2014, 5 (2), 205-209.

(25) Brown, P. J.; Muller, S., Open access chemical probes for epigenetic targets. Future Med. Chem. 2015, 7 (14), 1901-1917.

(26) Arrowsmith, C. H.; Audia, J. E.; Austin, C.; Baell, J.; Bennett, J.; Blagg, J.; Bountra, C.; Brennan, P. E.; Brown, P. J.; Bunnage, M. E.; Buser-Doepner, C.; Campbell, R. M.; Carter, A. J.; Cohen, P.; Copeland, R. A.; Cravatt, B.; Dahlin, J. L.; Dhanak, D.; Edwards, A. M.; Frye, S. V.; Gray, N.; Grimshaw, C. E.; Hepworth, D.; Howe, T.; Huber, K. V. M.; Jin, J.; Knapp, S.; Kotz, J. D.; Kruger, R. G.; Lowe, D.; Mader, M. M.; Marsden, B.; Mueller-Fahrnow, A.; Muller, S.; O'Hagan, R. C.; Overington, J. P.; Owen, D. R.; Rosenberg, S. H.; Roth, B.; Ross, R.; Schapira, M.; Schreiber, S. L.; Shoichet, B.; Sundstrom, M.; SupertiFurga, G.; Taunton, J.; Toledo-Sherman, L.; Walpole, C.; Walters, M. A.; Willson, T. M.; Workman, P.; Young, R. N.; Zuercher, W. J., The promise and peril of chemical probes. Nat. Chem. Biol. 2015, 11 (8), 536-541.

(27) Wagner, T.; Greschik, H.; Burgahn, T.; Schmidtkunz, K.; Schott, A. K.; McMillan, J.; Baranauskiene, L.; Xiong, Y.; Fedorov, O.; Jin, J.; Oppermann, U.; Matulis, D.; Schule, R.; Jung, M., Identification of a small-molecule ligand of the epigenetic reader protein Spindlin1 via a versatile screening platform. Nucleic Acids Res. 2016, 44 (9), e88.

(28) Machleidt, T.; Woodroofe, C. C.; Schwinn, M. K.; Mendez, J.; Robers, M. B.; Zirnmerman, K.; Otto, P.; Daniels, D. L.; Kirkland, T. A.; Wood, K. V., NanoBRET-a novel BRET platform for the analysis of protein-protein interactions. ACS Chem. Biol. 2015, 10 (8), 1797-1804.
(29) Niesen, F. H.; Berglund, H.; Vedadi, M., The use of differential scanning fluorimetry to detect ligand interactions that promote protein stability. Nat. Protoc. 2007, 2 (9), 2212-2221.

(30) Senisterra, G.; Zhu, H. Y.; Luo, X.; Zhang, H. L.; Xun, G. L.; Lug, C. L.; Xiao, W.; Hapan, T.; Loppnau, P.; Chau, I.; Li, F. L.; AllaliHassani, A.; Atadja, P.; Oyang, C.; Li, E.; Brown, P. J.; Arrowsmith, C. H.; Zhao, K. H.; Yu, Z. T.; Vedadi, M., Discovery of small-molecule antagonists of the H3K9me3 binding to UHRF1 tandem tudor domain. Slas Discov. 2018, 23 (9), 930-940.

(31) Drewes, G.; Knapp, S., Chemoproteomics and chemical probes for target discovery. Trends Biotechnol. 2018, 36 (12), 1275-1286.

(32) Valach, J.; Fik, Z.; Strnad, H.; Chovanec, M.; Plzak, J.; Cada, Z.; Szabo, P.; Sachova, J.; Hroudova, M.; Urbanova, M.; Steffl, M.; Paces, J.; Mazanek, J.; Vlcek, C.; Betka, J.; Kaltner, H.; Andre, S.; Gabius, H. J.; Kodet, R.; Smetana, K., Jr.; Gal, P.; Kolar, M., Smooth muscle actinexpressing stromal fibroblasts in head and neck squamous cell carcinoma: increased expression of galectin-1 and induction of poor prognosis factors. Int. J. Cancer 2012, 131 (11), 2499-2508.

(33) Heit, C.; Jackson, B. C.; McAndrews, M.; Wright, M. W.; Thompson, D. C.; Silverman, G. A.; Nebert, D. W.; Vasiliou, V., Update of the human and mouse SERPIN gene superfamily. Hum. Genomics 2013, 7, 22 .

(34) Izuhara, K.; Yamaguchi, Y.; Ohta, S.; Nunomura, S.; Nanri, Y.; Azuma, Y.; Nomura, N.; Noguchi, Y.; Aihara, M., Squamous cell carcinoma antigen 2 (SCCA2, SERPINB4): an emerging biomarker for skin inflammatory diseases. Int. J. Mol. Sci. 2018, 19 (4), 1102

(35) Kobashi, M.; Morizane, S.; Sugimoto, S.; Sugihara, S.; Iwatsuki, K., Expression of serine protease inhibitors in epidermal keratinocytes is increased by calcium but not 1,25-dihydroxyvitamin D-3 or retinoic acid. Brit. J. Dermatol. 2017, 176 (6), 1525-1532.

(36) Sivaprasad, U.; Kinker, K. G.; Ericksen, M. B.; Lindsey, M. Gibson, A. M.; Bass, S. A.; Hershey, N. S.; Deng, J. Y.; Medvedovic, M.; Hershey, G. K. K., SERPINB3/B4 contributes to early inflammation and barrier dysfunction in an experimental murine model of atopic dermatitis. J. Invest. Dermatol. 2015, 135 (1), 160-169.

(37) Sun, Y.; Sheshadri, N.; Zong, W. X., SERPINB3 and B4: From biochemistry to biology. Semin. Cell Dev. Biol. 2017, 62, 170-177.

(38) Wen, J.; Nikitakis, N. G.; Chaisuparat, R.; Greenwell-Wild, T.; Gliozzi, M.; Jin, W. W.; Adli, A.; Moutsopoulos, N.; Wu, T. X.; Warburton, G.; Wahl, S. M., Secretory leukocyte protease inhibitor (SLPI) expression and tumor invasion in oral squamous cell carcinoma. Am. J. Pathol. 2011, 178 (6), 2866-2878.

(39) Yang, T.; Liang, D. C.; Koch, P. J.; Hohl, D.; Kheradmand, F.; Overbeek, P. A., Epidermal detachment, desmosomal dissociation, and destabilization of corneodesmosin in Spink5(-/-) mice. Gene. Dev. 2004, 18 (19), 2354-2358

(40) Catanzaro, J. M.; Sheshadri, N.; Zong, W. X., SerpinB3/B4: mediators of Ras-driven inflammation and oncogenesis. Cell Cycle 2014, 13 (20), 3155-3156.

(41) Pietzsch, J., S100 proteins in health and disease. Amino Acids 2011, 41 (4), 755-760.

(42) Schonthaler, H. B.; Guinea-Viniegra, J.; Wculek, S. K.; Ruppen, I.; Ximenez-Embun, P.; Guio-Carrion, A.; Navarro, R.; Hogg, N.; Ashman, K.; Wagner, E. F., S100A8-S100A9 protein complex mediates psoriasis by regulating the expression of complement factor C3. Immunity 2013, 39 (6), 1171-1181.

(43) Xia, C.; Braunstein, Z.; Toomey, A. C.; Zhong, J. X.; Rao, X. Q., S100 proteins as an important regulator of macrophage inflammation. Front. Immunol. 2018, 8, 1908.

(44) Diakos, C. I.; Charles, K. A.; McMillan, D. C.; Clarke, S. J., Cancer-related inflammation and treatment effectiveness. Lancet Oncol. 2014, 15 (11), e493-e503.

(45) Shabani, F.; Farasat, A.; Mahdavi, M.; Gheibi, N., Calprotectin (S100A8/S100A9): a key protein between inflammation and cancer. Inflamm. Res. 2018, 67 (10), 801-812.

(46) Netea, M. G.; van de Veerdonk, F. L.; van der Meer, J. W. M.; Dinarello, C. A.; Joosten, L. A. B., Inflammasome-independent regulation of IL-1-family cytokines. Annu. Rev. Immunol. 2015, 33, 49-77.

(47) Kuang, C. M.; Fu, X.; Hua, Y. J.; Shuai, W. D.; Ye, Z. H.; Li, Y.; Peng, Q. H.; Li, Y. Z.; Chen, S.; Qian, C. N.; Huang, W.; Liu, R. Y., BST2 confers cisplatin resistance via NF-kappa B signaling in nasopharyngeal cancer. Cell Death Dis. 2017, 8, e2874.

(48) Marelli, G.; Sica, A.; Vannucci, L.; Allavena, P., Inflammation as target in cancer therapy. Curr. Opin. Pharmacol. 2017, 35, 57-65. 
(49) Guimaraes, D. P.; Oliveira, I. M.; de Moraes, E.; Paiva, G. R.; Souza, D. M.; Barnas, C.; Olmedo, D. B.; Pinto, C. E.; Faria, P. A.; Gallo, C. V. D.; Small, I. A.; Ferreira, C. G.; Hainaut, P., Interferon-inducible guanylate binding protein (GBP)-2: a novel p53-regulated tumor marker in esophageal squamous cell carcinomas. Int. J. Cancer 2009, 124 (2), 272279 .

(50) Wu, Y. Z.; Antony, S.; Hewitt, S. M.; Jiang, G. J.; Yang, S. X.; Meitzler, J. L.; Juhasz, A.; Lu, J. M.; Liu, H.; Doroshow, J. H.; Roy, K. Functional activity and tumor-specific expression of dual oxidase 2 in pancreatic cancer cells and human malignancies characterized with a novel monoclonal antibody. Int. J. Oncol. 2013, 42 (4), 1229-1238.

(51) Oh, S. H.; Kim, W. Y.; Lee, O. H.; Kang, J. H.; Woo, J. K.; Kim, J. H.; Glisson, B.; Lee, H. Y., Insulin-like growth factor binding protein-3 suppresses vascular endothelial growth factor expression and tumor angiogenesis in head and neck squamous cell carcinoma. Cancer Sci. 2012, 103 (8), 1259-1266.

(52) Baxter, R. C., IGF binding proteins in cancer: mechanistic and clinical insights. Nat. Rev. Cancer 2014, 14 (5), 329-341.

(53) Vidotto, A.; Polachini, G. M.; de Paula-Silva, M.; Oliani, S. M.; Henrique, T.; Lopez, R. V. M.; Cury, P. M.; Nunes, F. D.; Gois, J. F.; de Carvalho, M. B.; Leopoldino, A. M.; Tajara, E. H., Differentially expressed proteins in positive versus negative HNSCC lymph nodes. BMC Med. Genomics 2018, 11, 73.

(54) Fang, L. Y.; Wong, T. Y.; Chiang, W. F.; Chen, Y. L., Fatty-acidbinding protein 5 promotes cell proliferation and invasion in oral squamous cell carcinoma. J. Oral Pathol. Med. 2010, 39 (4), 342-348.

(55) Liu, B.; Huang, G.; Zhu, H.; Ma, Z.; Tian, X.; Yin, L.; Gao, X.; He, X., Analysis of gene coexpression network reveals prognostic significance of CNFN in patients with head and neck cancer. Oncol. Rep. 2019, 41, 2168-2180.

(56) Zhou, J. P.; Gao, Z. L.; Zhou, M. L.; He, M. Y.; Xu, X. H.; Tao, D. T.; Yang, C. C.; Liu, L. K., Snail interacts with Id 2 in the regulation of TNF-alpha-induced cancer cell invasion and migration in OSCC. Am. J. Cancer Res. 2015, 5 (5), 1680-1691.

(57) Monisha, J.; Roy, N. K.; Padmavathi, G.; Banik, K.; Bordoloi, D.; Khwairakpam, A. D.; Arfuso, F.; Chinnathambi, A.; Alahmadi, T. A.; Alharbi, S. A.; Sethi, G.; Kumar, A. P.; Kunnumakkara, A. B., NGAL is downregulated in oral squamous cell carcinoma and leads to increased survival, proliferation, migration and chemoresistance. Cancers 2018, 10 (7), 228

(58) Pidugu, V. K.; Wu, M. M.; Yen, A. H.; Pidugu, H. B.; Chang, K. W.; Liu, C. J.; Lee, T. C., IFIT1 and IFIT3 promote oral squamous cell carcinoma metastasis and contribute to the anti-tumor effect of gefitinib via enhancing p-EGFR recycling. Oncogene 2019, 38, 3232-3247.

(59) Aldinucci, D.; Colombatti, A., The inflammatory chemokine CCL5 and cancer progression. Mediat. Inflamm. 2014, 14, Article ID 292376.

(60) Wu, Y. C.; Shen, Y. C.; Chang, J. W. C.; Hsieh, J. J.; Chu, Y.; Wang, C. H., Autocrine CCL5 promotes tumor progression in esophageal squamous cell carcinoma in vitro. Cytokine 2018, 110, 94-103.

(61) Barbasiewicz, M.; Marciniak, K.; Fedorynski, M., Phase transfer alkylation of arylacetonitriles revisited. Tetrahedron Lett. 2006, 47 (23), 3871-3874.

(62) Chae, J., Practical demethylation of aryl methyl ethers using an odorless thiol reagent. Arch. Pharm. Res. 2008, 31 (3), 305-309.

(63) Magano, J.; Chen, M. H.; Clark, J. D.; Nussbaumer, T., 2(Diethylamino) ethanethiol, a new reagent for the odorless deprotection of aromatic methyl ethers. J. Org. Chem. 2006, 71 (18), 7103-7105.

(64) Keates, T.; Cooper, C. D. O.; Savitsky, P.; Allerston, C. K.; Phillips, C.; Hammarstrom, M.; Daga, N.; Berridge, G.; Mahajan, P.; Burgess-Brown, N. A.; Muller, S.; Graslund, S.; Gileadi, O., Expressing the human proteome for affinity proteomics: optimising expression of soluble protein domains and in vivo biotinylation. N. Biotechnol. 2012, 29 (5), 515-525.

(65) Vonrhein, C.; Flensburg, C.; Keller, P.; Sharff, A.; Smart, O.; Paciorek, W.; Womack, T.; Bricogne, G., Data processing and analysis with the autoPROC toolbox. Acta Crystallogr. D Biol. Crystallogr. 2011, 67, 293-302.

(66) Kabsch, W., XDS. Acta Crystallogr. D Biol. Crystallogr. 2010, $66,125-132$

(67) Evans, P., Scaling and assessment of data quality. Acta Crystallogr. D Biol. Crystallogr. 2006, 62, 72-82.

(68) Evans, P. R.; Murshudov, G. N., How good are my data and what is the resolution? Acta Crystallogr. D Biol. Crystallogr. 2013, 69, 1204 1214.
(69) Winn, M. D.; Ballard, C. C.; Cowtan, K. D.; Dodson, E. J.; Emsley, P.; Evans, P. R.; Keegan, R. M.; Krissinel, E. B.; Leslie, A. G. W.; McCoy, A.; McNicholas, S. J.; Murshudov, G. N.; Pannu, N. S.; Potterton, E. A.; Powell, H. R.; Read, R. J.; Vagin, A.; Wilson, K. S., Overview of the CCP4 suite and current developments. Acta Crystallogr. D Biol. Crystallogr. 2011, 67, 235-242.

(70) Adams, P. D.; Afonine, P. V.; Bunkoczi, G.; Chen, V. B.; Davis, I. W.; Echols, N.; Headd, J. J.; Hung, L. W.; Kapral, G. J.; GrosseKunstleve, R. W.; McCoy, A. J.; Moriarty, N. W.; Oeffner, R.; Read, R. J.; Richardson, D. C.; Richardson, J. S.; Terwilliger, T. C.; Zwart, P. H., PHENIX: a comprehensive Python-based system for macromolecular structure solution. Acta Crystallogr. D Biol. Crystallogr. 2010, 66, 213221.

(71) Emsley, P.; Lohkamp, B.; Scott, W. G.; Cowtan, K., Features and development of Coot. Acta Crystallogr. D Biol. Crystallogr. 2010, 66, 486-501.

(72) Huber, K. V. M.; Superti-Furga, G., Profiling of small molecules by chemical proteomics. Methods Mol. Biol. 2016, 1394, 211-218.

(73) Kim, D.; Landmead, B.; Salzberg, S. L., HISAT: a fast spliced aligner with low memory requirements. Nat. Methods 2015, 12 (4), 357 360 .

(74) Liao, Y.; Smyth, G. K.; Shi, W., featureCounts: an efficient general purpose program for assigning sequence reads to genomic features. Bioinformatics 2014, 30 (7), 923-930.

(75) Love, M. I.; Huber, W.; Anders, S., Moderated estimation of fold change and dispersion for RNA-seq data with DESeq2. Genome Biol. 2014, 15 (12), 550.

(76) Fabregat, A.; Jupe, S.; Matthews, L.; Sidiropoulos, K.; Gillespie, M.; Garapati, P.; Haw, R.; Jassal, B.; Korninger, F.; May, B.; Milacic, M.; Roca, C. D.; Rothfels, K.; Sevilla, C.; Shamovsky, V.; Shorser, S.; Varusai, T.; Viteri, G.; Weiser, J.; Wu, G. M.; Stein, L.; Hermjakob, H.; D'Eustachio, P., The reactome pathway knowledgebase. Nucleic Acids Res. 2018, 46, D649-D655. 


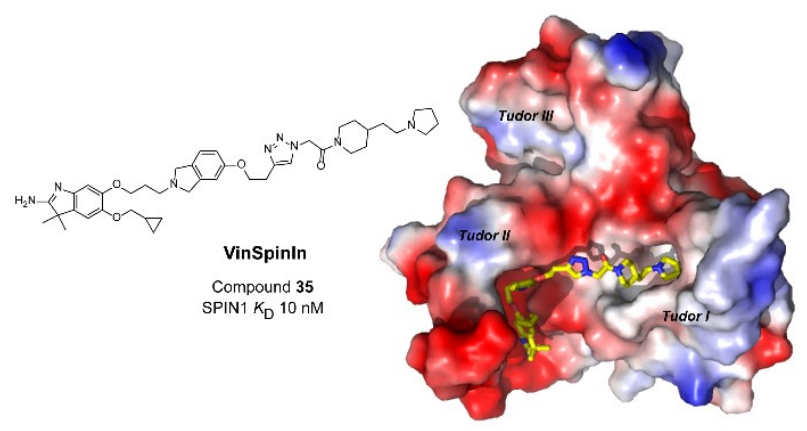

